Check for updates

Cite this: Phys. Chem. Chem. Phys., 2020, 22, 23952

Received 20th May 2020, Accepted 30th August 2020

DOI: $10.1039 / \mathrm{d} 0 \mathrm{cp} 02742 \mathrm{a}$

rsc.li/pccp

\section{A multiple decay-length extension of the Debye-Hückel theory: to achieve high accuracy also for concentrated solutions and explain under-screening in dilute symmetric electrolytes}

\author{
Roland Kjellander (iD
}

\begin{abstract}
The Poisson-Boltzmann and Debye-Hückel approximations for the pair distributions and mean electrostatic potential in electrolytes predict that these entities have one single decay mode with a decay length equal to the Debye length $1 / \kappa_{\mathrm{D}}$, that is, they have a characteristic contribution that decays with distance $r$ like $\mathrm{e}^{-\kappa \mathrm{D} r} / r$. However, in reality, electrolytes have several decay modes $\mathrm{e}^{-\kappa r} / r, \mathrm{e}^{-\kappa^{\prime} r} / r$ etc. with different decay lengths, $1 / \kappa, 1 / \kappa^{\prime}$ etc., that in general are different from the Debye length. As an illustration of the significance of multiple decay modes in electrolytes, the present work uses a very simple extension of the Debye-Hückel approximation with two decay lengths, which predicts oscillatory modes when appropriate. This approach gives very accurate results for radial distribution functions and thermodynamic properties of aqueous solutions of monovalent electrolytes for all concentrations investigated, including high ones. It is designed to satisfy necessary statistical mechanical conditions for the distributions. The effective dielectric permittivity of the electrolyte plays an important role in the theory and each mode has its own value of this entity. Electrolytes with high electrostatic coupling, like those with multivalent ions and/or with solvent of low dielectric constant, have decay lengths in dilute solutions that substantially deviate from the Debye length. It is shown that this is caused by nonlinear ion-ion correlation effects and the origin of under-screening, i.e., $1 / \kappa>1 / \kappa_{\mathrm{D}}$, in dilute symmetric electrolytes is analyzed. The under-screening is accompanied by an increase in the effective dielectric permittivity that is also caused by these correlations. The theoretical results for the decay length are successfully compared with recent experimental data for simple electrolytes in various solvents. The paper includes background material on electrolyte theory and screening in order to be accessible for nonexperts in the field.
\end{abstract}

\section{Introduction}

\subsection{Brief overview of electrolyte theories and screening}

Electrolytes are ubiquitous and in many fields of science and industrial applications it is important to understand their properties and to be able to calculate, for example, various thermodynamical and structural quantities. The study of electrolyte systems has a long history and is still a very active field of science. This illustrates both the importance and the complexity of such systems.

There exist an abundance of statistical mechanical theories for electrolytes that are based on approximations of various degrees of sophistication and accuracy. A selection of the most prominent ones that are relevant for bulk systems are presented and discussed in ref. 1-6. A cornerstone is the Debye-Hückel (DH) approximation, ${ }^{7}$ which gave an answer to

Department of Chemistry and Molecular Biology, University of Gothenburg, SE-412 96 Gothenburg, Sweden. E-mail: roland.kjellander@gu.se; Tel: +46 317869027 an important puzzle at the time of its development in the 1920s, namely experimentally observed deviations from ideality in very dilute electrolyte solutions. In solutions of nonelectrolytes such deviations disappear proportionally to the concentration, while for electrolytes they decay like the square root of concentration due to the long range $1 / r$ decay of the Coulomb interactions as function of distance $r$. In the DH approximation, the mean electrostatic potential from a charge is screened and decays like a Yukawa function $\mathrm{e}^{-\kappa_{\mathrm{D}} r} / r$, where $\kappa_{\mathrm{D}}$ is the is the Debye parameter and $1 / \kappa_{\mathrm{D}}$ the Debye length, which are obtained from

$$
\kappa_{\mathrm{D}}^{2}=\frac{\beta}{\varepsilon_{\mathrm{r}} \varepsilon_{0}} \sum_{j} n_{j} q_{j}^{2},
$$

where $\beta=1 / k_{\mathrm{B}} T, k_{\mathrm{B}}$ is Boltzmann's constant, $T$ is the absolute temperature, $\varepsilon_{\mathrm{r}}$ is the dielectric constant of the solvent, $\varepsilon_{0}$ is the permittivity of vacuum, $n_{j}$ is the number density and $q_{j}$ the charge of ions of species $j$ and the sum is taken over all species 
of ions in the system. The exponential screening leads to the Debye-Hückel limiting laws for the mean activity coefficient $\gamma_{ \pm}$ and the osmotic coefficient $\phi$, which are for a binary electrolyte

$$
\begin{aligned}
& \ln \gamma_{ \pm} \sim-\frac{\kappa_{\mathrm{D}} q_{+}\left|q_{-}\right|}{8 \pi \varepsilon_{\mathrm{r}} \varepsilon_{0} k_{\mathrm{B}} T} \\
& \phi-1 \sim-\frac{\kappa_{\mathrm{D}} q_{+}\left|q_{-}\right|}{24 \pi \varepsilon_{\mathrm{r}} \varepsilon_{0} k_{\mathrm{B}} T}
\end{aligned}
$$

in the limit of zero ionic density. These limiting laws and the existence of exponential screening for large $r$ are, in fact, exact features of statistical mechanics of dilute electrolytes.

The DH approximation is the linearized version of the Poisson-Boltzmann (PB) approximation, which is a mean field theory where the correlations between ions in the ion cloud around each ion are neglected. It gives a quite good description of electrolytes for low electrolyte concentrations, but fails when the concentration is increased. We here primarily consider the primitive model of electrolyte solutions, where the ions are charged hard spheres and the solvent is modeled as a dielectric continuum with dielectric constant $\varepsilon_{\mathrm{r}}$, and we will also treat classical plasmas of such ions for which $\varepsilon_{\mathrm{r}}=1$.

A large number of improvements of the $\mathrm{DH}$ approximation has been suggested over the years. One of the most well-known is the approach by Pitzer, ${ }^{8-10}$ where various $\mathrm{DH}$ expressions for thermodynamical quantities are parametrized to fit experimental data for a wide concentration interval. In most of the improved DH approximations, the decay length of the mean electric potential $\psi_{i}(r)$ from an ion of species $i$ is equal to the Debye length. The actual decay length in electrolytes is, however, in general not equal to the Debye length; it is only approximately equal to the Debye length for sufficiently low concentrations. For higher concentrations the decay length depends significantly on other system parameters than those that appear in eqn (1), for example the ion sizes.

When the electrolyte concentration is increased and the decay length starts to deviate noticeably from the Debye length, the mean electrostatic potential $\psi_{i}(r)$ decays for large $r$ like $\mathrm{e}^{-\kappa r} / r$ where $\kappa \neq \kappa_{\mathrm{D}}$. The radial charge density $\rho_{i}(r)$ around an ion and, in general, the pair distribution function $g_{i j}(r)$ also decay in this manner. The deviation of $\kappa$ from $\kappa_{\mathrm{D}}$ is not described by the $\mathrm{DH}$ or $\mathrm{PB}$ approximations and neither is the fact that these functions become oscillatory at high ionic densities. ${ }^{11,12}$ The latter feature was found by Kirkwood already in the $1930 \mathrm{~s}^{13,14}$ and later confirmed in other approaches by Martynov $^{15}$ and Outhwaite. ${ }^{16}$ The presence of such oscillations is connected to the fact that there exist several decay modes in electrolytes, that is, contributions to the distribution functions and the electrostatic potential that decay like Yukawa functions $\mathrm{e}^{-\kappa r} / r, \mathrm{e}^{-\kappa^{\prime} r} / r$ etc. where the decay parameters $\kappa, \kappa^{\prime}$ etc. are different. For low ionic densities, the two leading decay parameters, which give the longest decay lengths, satisfy $\kappa<\kappa^{\prime}$. When the ion density is further increased, $\kappa$ and $\kappa^{\prime}$ approach each other and at the so-called Kirkwood crossover point they become equal. Beyond that point $\kappa$ and $\kappa^{\prime}$ turn complex and we have $\kappa=\kappa_{\Re}+\mathrm{i} \kappa_{\mathfrak{T}}$ and $\kappa^{\prime}=\underline{\kappa}=\kappa_{\Re}-\mathrm{i} \kappa_{\Im}$, where $\mathrm{i}$ is the imaginary unit and $\kappa_{\Re}$ and $\kappa_{\mathfrak{I}}$ are real (underbar denotes complex conjugation). The pair of decay modes then gives rise to a contribution that decays like an "oscillatory Yukawa function" $\mathrm{e}^{-\kappa_{\Re} r} \cos \left(\kappa_{\Im} r+\alpha\right)$, where $\alpha$ gives the phase for the oscillations. This kind of oscillatory mode occurs in concentrated solutions and is the dominant one for molten salts.

The presence of several decay modes with different decay parameters has been numerically verified for simple electrolytes by computer simulations, ${ }^{17-20}$ the Hypernetted Chain (HNC) approximation $^{18,21-23}$ and the Generalized Mean Spherical Approximation (GMSA). ${ }^{24}$ The leading modes give rise to the oscillatory behavior at high ion densities in accordance with the scenery above. Many other approximate theories for electrolytes also predict such decay modes and the occurrence of the Kirkwood cross-over, for example the Mean Spherical Approximation (MSA), ${ }^{25-29}$ the Linearized Modified Poisson-Boltzmann (LMPB) approximation by Outhwaite, ${ }^{16,30,31}$ the Modified Debye-Hückel (MDH) approximation by Kjellander," the closely related "Local Thermodynamics" (LT) approximation by $\mathrm{Hall}^{33}$ the Generalized Debye-Hückel (GDH) approximation by Lee and Fisher, ${ }^{34,35}$ the Modified MSA by Varela and coworkers, ${ }^{36-39}$ the charge renormalization theory by Ding et $a l^{40}$ and the ionic cluster model approach by Avni and coworkers. ${ }^{41}$

These theories, from GMSA onwards, are linear approximations, meaning that $\psi_{i}(r)$ and $\rho_{i}(r)$ are proportional to the ionic charge $q_{i}$. They provide explicit equations for the decay parameter with multiple solutions $\kappa, \kappa^{\prime}$ etc. including complexvalued ones when appropriate and so does also the original theory by Kirkwood and that by Martynov. All give decay parameters that are dependent on the ionic diameter $a$ and qualitatively they give similar results for $\kappa / \kappa_{\mathrm{D}}$ and $\kappa^{\prime} / \kappa_{\mathrm{D}}$ as functions of $\kappa_{\mathrm{D}} a$. Most of these approximations have been used to obtain thermodynamical quantities, distribution functions and/or the mean electrostatic potential. ${ }^{15,16,27,28,31-33,37,39,42-47}$ Xiao and Song ${ }^{48-50}$ have developed a linear theory that exploits all decay modes obtained in many of these approximations to calculate thermodynamical quantities for electrolytes. We will return to some of these linear, approximate theories later. All of them work in general well for low ionic densities but have shortcomings, especially for high densities.

When the electrostatic coupling between the ions is not weak, linear theories are inadequate. Many nonlinear approximations for electrolytes have been used to obtain pair distributions and thermodynamical quantities. They include the HNC approximation $^{51-55}$ and other integral equation theories. ${ }^{54,56-61}$ There are also improved versions of the PB approximation that correct for the neglect of correlations in the ion cloud around each ion, like the Modified PB approximation by Outhwaite et al. ${ }^{16,62-64}$ and the correlation-enhanced $\mathrm{PB}$ theory by $\mathrm{Su}$ and coworkers. ${ }^{65}$ Furthermore, various field theories ${ }^{4,66-70}$ have been developed for the study of bulk electrolytes. Pair distributions and thermodynamical quantities for bulk electrolytes have, of course, been calculated also by simulations. ${ }^{11,12,17-20,55,59,61,71-80}$

The nonlinear theories do not in general provide any explicit equation for the decay parameter like the linear ones above do, but from these theories and from computer simulations, 
the values of $\kappa, \kappa^{\prime}$ etc. have been extracted numerically for various electrolytes. ${ }^{17-23,81-84}$ The decay parameter $\kappa$ of the leading decay mode can in many cases be obtained by curve fitting to the tails of the functions for large $r$, but it is in general not straightforward to obtain those of the other modes and one must use systematic means to extract them from the distribution functions. ${ }^{18}$

One approximate way to incorporate some effects of nonlinearity into linear theories is to introduce ion pairing as originally done by Bjerrum. ${ }^{85}$ In this approach one assumes that anion-cation pairs exist in equilibrium with free ions in the electrolyte, so the concentration of the latter ions is lower than the total concentration. Since the pairs are electroneutral, they do not contribute to the Debye parameter in eqn (1) and the Debye length $1 / \kappa_{\mathrm{D}}$ becomes larger than in their absence - a quite trivial reason for a change in decay length. The pair formation is a nonlinear phenomenon because the electrostatic interaction between two paired ions is large. The effects of this nonlinearity is included in the value of the equilibrium constant that describes the equilibrium.

There exist various ways to construct theories of pairing. One kind of approach is to include ion pairs in a DH-type theory ${ }^{86-88}$ for the electrolyte and another is to base the ion pair theory on the MSA; ${ }^{8-92}$ see ref. 93 for a comparison of different variants of these approaches. Fisher and coworkers ${ }^{34,88,93}$ stress the importance of including the interactions between the dipoles formed by the ion pairs and the free ions. In many cases, it is reasonable to consider a part of the effect from the presence of such dipoles as an augmentation of the dielectric permittivity of the electrolyte. We will treat some aspects of ion pairing and changes in dielectric permittivity later. Transient ion pairing is, in fact, an aspect of strong anion-cation correlations, which affect the decay length and the (effective) dielectric permittivity of the electrolyte. ${ }^{94,95}$

Electrolyte systems have in general a complex behavior and most theories mentioned above are quite complicated to use, in particular those that yield accurate distribution functions. A fair question to ask is how thermodynamic properties and distribution functions possibly can be accurately obtained without undue complications and without the use of empirical fitting parameters. In order a get a perspective on this question, let us first consider some approaches that have been used for such a task.

A simple linear approximation that takes into consideration that the decay length depends on the ion diameter is the Corrected Debye-Hückel (CDH) approximation by Abbas, Nordholm and coworkers. ${ }^{78,96}$ They make an Ansatz that the radial charge density $\rho_{i}(r)$ around an ion (and therefore the electrostatic potential $\left.\psi_{i}(r)\right)$ is proportional to $\mathrm{e}^{-\kappa r} / r$, where the decay parameter $\kappa$ is not the same as $\kappa_{\mathrm{D}}$. The value of $\kappa$ is then determined from an optimization of an approximate free energy of the system. By combining $\mathrm{CDH}$ with the Carnahan-Starling equation of state $^{97}$ for hard spheres, they obtain the activity coefficient for monovalent $(1: 1)$ electrolytes in aqueous solution in very good agreement with simulations for a wide range of concentrations, including concentrated solutions. ${ }^{78}$ For 2:1 electrolytes they obtain equally good agreement except for small ion sizes, but the latter results are still good. As pointed out by Abbas et al.,$^{78}$ the thermodynamical quantities obtained from this Ansatz are in very good agreement with simulation results for high densities despite that the actual $\rho_{i}(r)$ is oscillatory rather than proportional to $\mathrm{e}^{-\kappa r} / r$.

Likewise, for a parametrized $\mathrm{DH}$ approach with fitting parameters for thermodynamics like that of Pitzer mentioned earlier, the structural entities are qualitatively different from the accurate ones at high concentrations. The same is the case for approaches that use the PB approximation.

Attard $^{84}$ also makes the assumption that $\rho_{i}(r)$ is proportional to $\mathrm{e}^{-\kappa r} / r$. By requiring that $\rho_{i}(r)$ fulfills some necessary conditions that we will consider later, he obtains the Self-Consistent Screening Length (SCSL) approximation for the single decay parameter $\kappa$, which can be a real or complex number depending on the ion density. This is a linear theory that has been successfully used to calculate thermodynamical quantities for monovalent electrolytes solutions with low up to moderate concentrations. Another theory that uses only one decay mode in the calculation of thermodynamical properties is the MDH approximation. ${ }^{32}$ It is also successful for low up to moderate concentrations. These two approximations will be treated in quite some detail later.

Janecek and Netz ${ }^{80}$ investigated whether properties of electrolytes can be described in terms of an effective screening length. They extracted a single effective screening parameter $\kappa_{\text {eff }}$ by simulation of various electrolyte solutions (corresponding to $1: 1$ and $2: 2$ salt in water) and used it to calculate thermodynamic properties from a DH-like theory where $\kappa_{\mathrm{D}}$ is replaced by $\kappa_{\text {eff. }}$ For high concentrations where the simulated radial charge density is oscillatory, a complex-valued screening parameter was extracted instead of $\kappa_{\text {eff. This approach works }}$ very well for the thermodynamics of 1:1 electrolytes for the concentration range investigated and somewhat less well for the $2: 2$ case. The parameter $\kappa_{\text {eff }}$ is in general not the same as the decay parameter $\kappa$ of the asymptotic decay of $\rho_{i}(r)$ discussed in the current work, $\dagger$ but $\kappa_{\text {eff }}$ approaches the actual $\kappa$ when the concentration is decreased towards zero. As pointed out by the authors, the effective screening parameter "does not provide an accurate description of the charge density distribution." They also used the ion-pairing DH-type theory by Fisher and coworkers $^{88,93}$ to calculate activity coefficients, which worked very well for $2: 2$ electrolytes and less well for monovalent ones.

From these examples we see that even if an approximation reproduces thermodynamical data accurately, the structural entities like $\rho_{i}(r)$ and the pair distribution function $g_{i j}(r)$ obtained in the approximation do not need to be equal to the correct ones. Even when these functions are oscillatory, the thermodynamical entities can be accurately obtained from a

$\dagger$ In fact, $\kappa_{\text {eff }}$ is evaluated in ref. 80 from the simulations via a route that implies that $\kappa_{\text {eff }}$ satisfies Attard's approximate SCSL $^{84}$ equation for $\kappa$ [eqn (38) in the current work]. This is the reason why $\kappa_{\text {eff }}$ from the simulations agrees with $\kappa$ from the SCSL approximation; see the authors' remark about this agreement in the text below eqn (47) in ref. 80. Any difference between the two values is due numerical noise in the simulation. 
single Yukawa decay mode with a real decay parameter. Likewise, when there are more than one decay mode, the thermodynamics can be accurately obtained from just one Yukawa mode. The value of the decay parameter used is in general different from that of the actual $\kappa$ of the electrolyte. To judge whether an approximation gives a fully accurate description of a system, it is accordingly important to investigate both thermodynamical and structural quantities. A successful theory gives accurate results for both. An important aim of the current work is to develop a simple theory that accomplishes this. Thereby, the presence of more than one decay mode will be included in the treatment of simple electrolytes.

Incidentally, it should be noted that several simultaneous modes that decay like monotonic or oscillatory Yukawa functions have also a prominent role in dense ionic liquids, like room temperature ionic liquids. An analysis of these decay modes is important for the understanding of such systems not only for structure and thermodynamics, but also for interactions in the systems including surface forces. The modes can, for example, be detected in surface force experiments. It has recently been shown ${ }^{95,98}$ that all such decay modes, including those that are dominated by "packing" of molecules in the dense liquids, are in general also decay modes of the electrostatic interactions and are therefore governed by the dielectric response of the liquid. $\neq$ The same is true also for the decay mode of long-range density-density correlation fluctuations with a decay length that diverges on approach to a critical point and the very long-range monotonically decaying mode that recently has been found ${ }^{99}$ for dense electrolytes. Likewise, for dilute electrolyte solutions with discrete molecular solvent, oscillatory decay modes that are dominated by the structure of the solvent also constitute decay modes of the electrostatic interactions. ${ }^{95,98}$ Such modes have often been designated as "solvation forces;" in the case of aqueous systems "hydration forces."

\subsection{Overview of the current work}

We will limit ourselves in the current work to solutions of simple electrolytes in the primitive model and to classical plasmas. In order to illustrate the importance of the various decay modes, we will include two Yukawa modes and show that both modes are crucial in order to obtain very good description of both structural and thermodynamical quantities for a wide range of ion densities, including concentrated solutions and dense plasmas. As concluded in the previous section, a successful theory accurately predicts both kinds of quantities. The decay modes are physically distinguishable and the decay lengths $1 / \kappa$ and $1 / \kappa^{\prime}$ constitute characteristic properties of the propagation of screened electrostatic interactions in the electrolyte. For the oscillatory case, where $\kappa$ and $\kappa^{\prime}$ are complex, the decay length $1 / \kappa_{\Re}$ and the wave length $2 \pi / \kappa_{\mathfrak{I}}$ have the corresponding roles.

‡ An exception is charge-inversion invariant systems, for example, the restricted primitive model where anions and cations are identical apart from their sign of charge. In real systems, the anions and cations differ by more than the signs of their charges, so the systems are not charge-inversion invariant.
The basic idea in this approach is simple: for a radial function $f(r)$ like $\psi_{i}(r), \rho_{i}(r)$ or the potential of mean force $w_{i j}(r)$ between two ions, one makes the approximate Ansatz that $f(r)$ is given by

$$
f(r)=C \frac{\mathrm{e}^{-\kappa r}}{r}+C^{\prime} \frac{\mathrm{e}^{-\kappa^{\prime} r}}{r}
$$

for all $r$ outside the hard core of the ion. The coefficients $C$ and $C^{\prime}$ and the decay parameters $\kappa$ and $\kappa^{\prime}$ are then determined from necessary statistical mechanical conditions for the radial function in question. Moreover, the coefficients are expressed in terms of physical entities like effective charges of ions and effective permittivities of the electrolyte - entities that are precisely defined in exact statistical mechanics as explained later. These entities are important for the understanding of screening phenomena and intermolecular interactions in electrolytes and the current paper also serves the purpose of illustrating their meaning and use. The simple structure of the approximations used in this work facilitates this objective.

An important further ingredient is the use of an exact relationship§ between the decay parameter $\kappa$ and the effective charges, namely ${ }^{100,101}$

$$
\kappa^{2}=\frac{\beta}{\varepsilon_{\mathrm{r}} \varepsilon_{0}} \sum_{j} n_{j} q_{j} q_{j}^{\mathrm{eff}},
$$

where $q_{j}^{\text {eff }}$ is the effective charge of a $j$-ion. This relationship, which is valid for a system with spherical ions, is similar to the definition of $\kappa_{\mathrm{D}}$ in eqn (1), but it has the product $q_{j} q_{j}^{\text {eff }}$ instead of $q_{j}^{2}$ in the right hand side (rhs). In the next section we will see in a simple manner that eqn (4) is a direct consequence of the ion-ion correlations in the ion cloud around each ion. When such correlations are neglected like in the DH and PB approximations, we have $q_{j}^{\text {eff }}=q_{j}$ for the ions in the cloud and eqn (1) for the Debye parameter is obtained.

In the present work, we will primarily investigate systems with sufficiently low electrostatic coupling so that electrostatic response can be linearized. The resulting mathematical formulas for both the structural and thermodynamic quantities are simple and straight-forward to apply in practice. They are obtained by using first-principle statistical mechanics and are free from any fitting parameters. The results obtained for conditions corresponding to monovalent ions in aqueous solutions at room temperature are in very good agreement with computer simulations and other accurate calculations for all concentrations. Such conditions also include classical plasmas at high temperatures. We will also see why it is insufficient with one single decay mode for the accurate description of distribution functions of electrolytes with medium to high densities.

Nonlinear correlational effects for high electrostatic coupling will also be investigated. In fact, the long-range nature of the Coulomb interactions gives such effects in highly diluted electrolyte solutions and thin plasmas. They can cause substantial deviations of $\kappa$ from $\kappa_{\mathrm{D}}$, which are given by the

§ In this work "exact relationship," "exact equation" etc. mean that they are derived without any approximations in statistical mechanics for a given model, that is, for a given Hamiltonian that defines the system. 
following exact limiting law ${ }^{101}$ - here written for the case of a binary electrolytes $\Phi$

$$
\begin{aligned}
\frac{\kappa^{2}}{\kappa_{\mathrm{D}}^{2}} \sim 1 & +\frac{\Lambda \ln 3}{4}\left(z_{+}-\left|z_{-}\right|\right)^{2} \\
& +\frac{\Lambda^{2} \ln \Lambda}{6}\left(z_{+}^{2}-z_{+}\left|z_{-}\right|+z_{-}^{2}\right)^{2} \quad \text { when } \Lambda \rightarrow 0,
\end{aligned}
$$

where $\Lambda=\ell_{\mathrm{B}} \kappa_{\mathrm{D}}$ is a coupling constant, $\ell_{\mathrm{B}}=\beta q_{\mathrm{e}}{ }^{2} /\left(4 \pi \varepsilon_{\mathrm{r}} \varepsilon_{0}\right)$ is the Bjerrum length, $q_{\mathrm{e}}$ is the elementary (protonic) charge and $z_{j}=q_{j} / q_{\mathrm{e}}$ is the ionic valency. The deviation of $\kappa^{2} / \kappa_{\mathrm{D}}^{2}$ from the value 1 , as described by this law, arises solely from the tails of the long-range, purely electrostatic correlations between the ions when $r \rightarrow \infty$ and does not depend on any other properties of the ions than their charges. These other properties, like the ion size, enter in the following term (not shown in eqn (5)) that is proportional to $\Lambda^{2}$, i.e., proportional to the ionic concentration.

For symmetric electrolytes $\left(z_{+}=\left|z_{-}\right|\right)$, the second term in the rhs of eqn (5) is identically equal to zero and we have $\kappa<\kappa_{\mathrm{D}}$ for sufficiently dilute solutions because $\ln A<0$ there, which means that the decay length is larger than the Debye length ("under-screening"). For dilute solutions of asymmetric electrolytes $\left(z_{+} \neq\left|z_{-}\right|\right)$, the second term is dominant and for such electrolytes $\kappa>\kappa_{\mathrm{D}}$ in the limit of infinite dilution, so the decay length is smaller than the Debye length ("over-screening").

These predictions have been verified experimentally. For a highly asymmetric electrolyte, $z_{+}=12$ and $z_{-}=-1$, the positive deviation of $\kappa$ from $\kappa_{\mathrm{D}}$ has been experimentally measured in dilute solutions by Kékicheff and Ninham ${ }^{103}$ in agreement with the leading term in eqn (5). For symmetric electrolytes, the negative deviation of $\kappa$ from $\kappa_{\mathrm{D}}$, has recently been measured in dilute solutions of simple electrolytes with high electrostatic coupling by Smith, Maroni, Trefalt and Borkovec ${ }^{104}$ and Stojimirović, Galli and Trefalt ${ }^{105}$ (henceforth referred to as "Trefalt and coworkers") by surface force experiments. This latter case will be discussed in quite some detail in the current work.

While all linear approximations mentioned earlier give the correct limiting laws for the thermodynamical properties, eqn (2), in the limit of zero ionic density, they do not give the correct behavior of $\kappa^{2} / \kappa_{\mathrm{D}}^{2}$ in this limit as given by eqn (5). For example, the MSA, GMSA, LMPB, MDH, LT and GDH approximations predict that $\kappa^{2} / \kappa_{\mathrm{D}}^{2}$ decays proportionally to $\Lambda^{2}$ when the density goes to zero. On the other hand, the HNC approximation, which is a nonlinear approximation, gives a $\kappa$ that agrees with eqn (5) for both symmetric ${ }^{22}$ and asymmetric $^{81}$ electrolytes at low ionic densities. In the analysis presented in the current work, we will see the importance of including nonlinear ion-ion correlation effects in order to obtain the correct behavior of the decay parameter for dilute systems. Such effects influence the values the effective ionic charges and hence $\kappa$ via eqn (4).

T The term proportional to $\Lambda$ in eqn (5) was originally derived in ref. 102 .
The paper is organized as follows. In Section 2, some relevant parts of the theory of electrolytes are presented as a background for the rest of the paper and the main approximations made in the current work are introduced. A couple of approximations with a single decay-mode are treated in Section 3 and a simple equation for $\kappa$ that is used in a large part of the paper is obtained. The Multiple-Decay Extended Debye-Hückel (MDE-DH) approximation, developed in this work, is described in Section 4, starting with the simplest version, which is sufficient provided that the ionic sizes are not too large so ionic core-core correlations are not too prominent. This version of the theory gives both radial distribution functions and thermodynamical quantities in very good agreement with simulations and Hypernetted Chain (HNC) calculations that are very accurate for the systems studied. The complete version of the MDE-DH approximation is used to successfully treat dense systems with large ions, whereby quite intricate details of the pair distribution functions are obtained in agreement with simulations. In Section 5, effects of nonlinear ion-ion correlations on the main decay mode are investigated. The screening properties of thin plasmas and dilute solutions of symmetric electrolytes are thereby studied, in particular the underscreening that occurs in such electrolytes with high electrostatic coupling. This under-screening is accompanied by an augmentation of the effective dielectric permittivity of the electrolyte caused by the strongly nonlinear ion-ion correlations. The various types of deviations of $\kappa$ from $\kappa_{\mathrm{D}}$ in symmetric electrolytes and the reasons for them are studied in Section 6 , whereby an approximate limiting law for $\kappa^{2} / \kappa_{\mathrm{D}}{ }^{2}$ is derived for symmetric electrolytes that extends the exact law (5) by adding higher order contributions. An analysis is made of the experimental results by Trefalt and coworkers ${ }^{104,105}$ for the deviations of $\kappa$ from $\kappa_{\mathrm{D}}$ in dilute solutions of simple electrolytes with high electrostatic coupling. Different mechanisms for this phenomenon are discussed. Finally, in Section 7 the main results of the paper are summarized and concluding remarks are given. As an aid to the reader, this section contains references to some central equations of this work.

\section{Background and basic approximations}

We will consider electrolytes in bulk phase using the primitive model. This model is also applicable to classical plasmas where $\varepsilon_{\mathrm{r}}=1$. Each ion has a point charge $q_{j}$ at its center and the total ion density in bulk is $n_{\mathrm{tot}}=\sum_{j} n_{j}$.

The average density of ions of species $j$ at distance $r$ from the center of an ion of species $i$ is equal to $n_{j} g_{i j}(r)=n_{j} \mathrm{e}^{-\beta w_{i j}(r)}$, where $g_{i j}(r)$ is the pair distribution function and $w_{i j}(r)$ is the pair potential of mean force. The mean charge density around the $i$-ion is given by the radial charge-distribution function

$$
\rho_{i}(r)=\sum_{j} q_{j} n_{j} g_{i j}(r)=\sum_{j} q_{j} n_{j} h_{i j}(r)
$$


where $h_{i j}(r)=g_{i j}(r)-1$ and the last equality follows from the electroneutrality condition $\sum_{j} q_{j} n_{j}=0$ for the bulk phase. When dealing with $\rho_{i}(r)$ and other functions of $r$, we will denote this $i$-ion as the "central ion" and the surrounding charge density constitutes its ion cloud.

In the Poisson-Boltzmann approximation one assumes that

$$
w_{i j}(r)=q_{j} \psi_{i}(r) \quad(\mathrm{PB})
$$

for $r \geq a$, where $\psi_{i}(r)$ is the mean electrostatic potential from the ionic charge $q_{i}$ and the surrounding charge density $\rho_{i}(r)$. The notation "(PB)" on the equation means that the equation is applicable in the PB approximation only. The potential $\psi_{i}(r)$ decays like

$$
\psi_{i}(r) \sim C_{i} \frac{\mathrm{e}^{-\kappa_{\mathrm{D}} r}}{r} \quad \text { when } r \rightarrow \infty \quad(\mathrm{PB})
$$

where $C_{i}$ is a constant. In the limit of infinite dilution $\kappa_{\mathrm{D}} \rightarrow 0$, $C_{i} \rightarrow q_{i} /\left(4 \pi \varepsilon_{\mathrm{r}} \varepsilon_{0}\right)$ and $\psi_{i}(r)$ approaches the ordinary Coulombic potential $q_{i} /\left(4 \pi \varepsilon_{\mathrm{r}} \varepsilon_{0} r\right)$ from the charge $q_{i}$. Therefore, for an electrolyte of any ionic density, it is reasonable to define an effective charge $q_{i}^{\text {eff }}$ of the ion from $C_{i}=q_{i}^{\text {eff }} /\left(4 \pi \varepsilon_{\mathrm{r}} \varepsilon_{0}\right)$ in the PB approximation, so we have

$$
\psi_{i}(r) \sim \frac{q_{i}^{\text {eff }}}{4 \pi \varepsilon_{\mathrm{r}} \varepsilon_{0}} \cdot \frac{\mathrm{e}^{-\kappa_{\mathrm{D}} r}}{r} \quad \text { when } r \rightarrow \infty \quad(\mathrm{PB}),
$$

where $q_{i}^{\text {eff }} \neq q_{i}$. One can say that $q_{i}^{\text {eff }}$ is the charge that the ion is perceived to have when seen from a distance, that is, as judged from the magnitude of the large $r$ tail of the electrostatic potential. The magnitude of the tail is affected by the amount of charge in the ion cloud close to the central ion and the value of $q_{i}^{\text {eff }}$ is determined by the distribution of charge there. Since $\psi_{i}(r)$ varies in a nonlinear manner with $q_{i}$, the effective charge also varies nonlinearly with $q_{i}$. It follows from eqn (7) that in the PB approximation we have

$$
w_{i j}(r) \sim \frac{q_{i}^{\mathrm{eff}} q_{j}}{4 \pi \varepsilon_{\mathrm{r}} \varepsilon_{0}} \cdot \frac{\mathrm{e}^{-\kappa_{\mathrm{D}} r}}{r} \quad \text { when } r \rightarrow \infty \quad(\mathrm{PB}),
$$

which does not obey the required symmetry $w_{i j}=w_{j i}$, a wellknown deficiency of this approximation. The reason for this deficiency is that the ions in the ion cloud are treated in a different manner than the central ion. The former are treated as point ions that do not correlate with each other. They only correlate with the central ion, which is the only ion in the system that has an ion cloud of its own and therefore an effective charge $q_{i}^{\text {eff }} \neq q_{i}$. All ions apart from the central ion enter in $w_{i j}$ with their actual (bare) charge $q_{j}$. This applies also to those of the same species as the central ion.

In the linearized version of the $\mathrm{PB}$ approximation, the Debye-Hückel approximation, it is assumed that $\beta w_{i j}(r)$ is sufficiently small so that $\mathrm{e}^{-\beta w_{i j}(r)} \approx 1-\beta w_{i j}(r)$ and the following well-known expressions are valid for $r \geq a$

$$
\psi_{i}(r)=\frac{q_{i} \mathrm{e}^{\kappa_{\mathrm{D}} a}}{4 \pi \varepsilon_{\mathrm{r}} \varepsilon_{0}\left(1+\kappa_{\mathrm{D}} a\right)} \cdot \frac{\mathrm{e}^{-\kappa_{\mathrm{D}} r}}{r}(\mathrm{DH})
$$

$$
\rho_{i}(r)=-\frac{\kappa_{\mathrm{D}}^{2} q_{i} \mathrm{e}^{\kappa_{\mathrm{D}} a}}{4 \pi\left(1+\kappa_{\mathrm{D}} a\right)} \cdot \frac{\mathrm{e}^{-\kappa_{\mathrm{D}} r}}{r}(\mathrm{DH}) .
$$

By making the identification of $q_{i}^{\text {eff }}$ given by eqn (8) we obtain

$$
q_{i}^{\text {eff }}=q_{i} \frac{\mathrm{e}^{\kappa_{\mathrm{D}} a}}{1+\kappa_{\mathrm{D}} a} \quad(\mathrm{DH}),
$$

so we can write for $r \geq a$

$$
\begin{gathered}
\psi_{i}(r)=\frac{q_{i}^{\text {eff }}}{4 \pi \varepsilon_{\mathrm{r}} \varepsilon_{0}} \cdot \frac{\mathrm{e}^{-\kappa_{\mathrm{D}} r}}{r}(\mathrm{DH}) \\
\rho_{i}(r)=-\frac{\kappa_{\mathrm{D}}^{2} q_{i}^{\text {eff }}}{4 \pi} \cdot \frac{\mathrm{e}^{-\kappa_{\mathrm{D}} r}}{r} \quad(\mathrm{DH}) .
\end{gathered}
$$

Note that in this case, $q_{i}^{\text {eff }}$ is proportional to $q_{i}$ and so are $\psi_{i}$ and $\rho_{i}$. In the DH approximation we have $g_{i j}(r)=1-\beta w_{i j}(r)$ with $w_{i j}(r)$ equal to the rhs of eqn (9) for all $r \geq a$.

The total charge density associated with an $i$-ion, including the charge of the ion itself, is $\rho_{i}^{\text {tot }}(r)=q_{i} \delta^{(3)}(r)+\rho_{i}(r)$, where $\delta^{(3)}(r)$ is the three-dimensional Dirac delta function. The charge density must satisfy the condition of local electroneutrality $\int \mathrm{d} \mathbf{r} \rho_{i}^{\text {tot }}(r)=q_{i}+\int \mathrm{d} \mathbf{r} \rho_{i}(r)=0$, which is satisfied for $\rho_{i}$ in eqn (11) and (14). The electrostatic potential is given by the solution to Poisson's equation $-\varepsilon_{\mathrm{r}} \varepsilon_{0} \nabla^{2} \psi_{i}(r)=\rho_{i}^{\text {tot }}(r)$ and can be written as

$$
\psi_{i}(r)=\int \mathrm{d} \mathbf{r}^{\prime} \rho_{i}^{\mathrm{tot}}\left(r^{\prime}\right) \phi_{\mathrm{Coul}}\left(\left|\mathbf{r}-\mathbf{r}^{\prime}\right|\right)
$$

where $\phi_{\text {Coul }}=1 /\left(4 \pi \varepsilon_{\mathrm{r}} \varepsilon_{0} r\right)$ is the (unit) Coulomb potential and the integral is taken over the whole space (this is the convention throughout the paper for integrals without limits).

The (unit) screened Coulomb potential in electrolytes can be defined from the potential $\psi_{q}(r)$ from a point charge $q$ in the limit of zero $q$ in the following manner $\|$

$$
\phi_{\text {Coul }}{ }^{*}(r)=\lim _{q \rightarrow 0} \frac{\psi_{q}(r)}{q} .
$$

In the $\mathrm{PB}$ and $\mathrm{DH}$ approximations we have

$$
\phi_{\text {Coul }}{ }^{*}(r)=\frac{1}{4 \pi \varepsilon_{\mathrm{r}} \varepsilon_{0}} \cdot \frac{\mathrm{e}^{-\kappa_{\mathrm{D}} r}}{r} \quad(\mathrm{~PB}, \mathrm{DH}) .
$$

This function governs the spatial propagation of electrostatic potentials in the electrolyte. In the $\mathrm{DH}$ approximation we have $\psi_{i}(r)=q_{i}^{\text {eff }} \phi_{\text {Coul }}{ }^{*}(r)$ for $r \geq a$ and in the PB case $\psi_{i}(r) \sim q_{i}^{\text {eff }} \phi_{\text {Coul }^{*}}(r)$ for large $r$.

Also in the general (exact) case, $\phi_{\text {Coul }^{*}}(r), w_{i j}(r)$ and the other functions decay like a Yukawa function if the density is not too high, but the decay parameter $\kappa$ deviates from $\kappa_{\mathrm{D}}$ and the prefactors have other values. In exact statistical mechanics, all ions are treated on the same basis, which means that $w_{i j}$ is symmetrical with respect to $i$ and $j$ and all ions have an effective charge $q_{l}^{\text {eff }} \neq q_{l}$ for $l=i, j$. Dressed Ion Theory (DIT), ${ }^{22,23,100,101}$ which is an exact reformulation of classical, equilibrium statistical mechanics of electrolytes in the fluid state, gives a formalism to handle these matters. Provided that the ion

$\|$ The function $\phi_{\text {Coul }}{ }^{*}(r)$ is, in fact, a Green's function for spatial propagation of electrostatic field the electrolyte,$^{98}$ which is the proper way to define it. 
density is not too high we have from DIT

$$
\phi_{\text {Coul }}{ }^{*}(r) \sim \frac{1}{4 \pi \mathcal{E}_{\mathrm{r}}^{\mathrm{eff}} \varepsilon_{0}} \cdot \frac{\mathrm{e}^{-\kappa r}}{r} \quad \text { when } r \rightarrow \infty,
$$

where $\mathcal{E}_{\mathrm{r}}^{\text {eff }}$ is an effective dielectric permittivity of the electrolyte that differs from the dielectric constant $\varepsilon_{\mathrm{r}}$ of the solvent. Thus $1 / \mathcal{E}_{\mathrm{r}}^{\text {eff }}$ specifies the magnitude of the tail of $\phi_{\text {Coul }}{ }^{*}(r)$ for large $r$ and gives a measure of the dielectric response of the electrolyte. In the $\mathrm{PB}$ and $\mathrm{DH}$ approximations one sets $\mathcal{E}_{\mathrm{r}}^{\mathrm{eff}}$ equal to $\varepsilon_{\mathrm{r}}$, so the dielectric constant of the pure solvent is used in $\phi_{\mathrm{Coul}^{*}}{ }^{*}$ these approximations instead $\mathcal{E}_{\mathrm{r}}^{\mathrm{eff}}$, which is of a property of the electrolyte.

The mean electrostatic potential due to an $i$-ion decays like

$$
\psi_{i}(r) \sim \frac{q_{i}^{\text {eff }}}{4 \pi \mathcal{E}_{\mathrm{r}}^{\text {eff }} \varepsilon_{0}} \cdot \frac{\mathrm{e}^{-\kappa r}}{r} \quad \text { when } r \rightarrow \infty
$$

which is in analogy with the $\mathrm{PB}$ and $\mathrm{DH}$ approximations, but $q_{i}^{\text {eff }}$ has a different value and we have $\mathcal{E}_{\mathrm{r}}^{\text {eff }}$ in the denominator instead of $\varepsilon_{\mathrm{r}}$. Note that $\psi_{i}(r) \sim q_{i}^{\text {eff }} \phi_{\mathrm{Coul}^{*}}{ }^{*}(r)$ like in the $\mathrm{PB}$ approximation. Furthermore,

$$
w_{i j}(r) \sim \frac{q_{i}^{\text {eff }} q_{j}^{\text {eff }}}{4 \pi \mathcal{E}_{\mathrm{r}}^{\text {eff }} \varepsilon_{0}} \cdot \frac{\mathrm{e}^{-\kappa r}}{r} \quad \text { when } r \rightarrow \infty
$$

which is symmetrical in $i$ and $j$ as it must. Note that the decay of $h_{i j}(r)$ for large $r$ is the same as for $-\beta w_{i j}(r)$ because $h_{i j}(r)=\mathrm{e}^{-\beta w_{i j}(r)}$ $-1 \sim-\beta w_{i j}(r)$ when $\beta w_{i j}(r)$ is small. These asymptotic formulas constitute facts that are obtained from exact statistical mechanics for electrolytes. DIT provides methods to calculate $q_{l}^{\text {eff }}$ and $\mathcal{E}_{\mathrm{r}}^{\mathrm{eff}}$ from the distribution functions of the system, ${ }^{* *}$ but we will not enter into any details here.

The appearance of the product $q_{i}^{\text {eff }} q_{j}^{\text {eff }}$ in $w_{i j}(r)$ of eqn (18) has some immediate, important consequences. Using eqn (17) we see that $w_{i j}(r) \sim q_{j}^{\text {eff }} \psi_{i}(r)$ for large $r$, so the effective charge appears as the prefactor instead of the actual charge $q_{i}$ that one has in the PB approximation $w_{i j}(r)=q_{j} \psi_{i}(r)$. Recall that the latter equation caused the incorrect symmetry of eqn (9). Since $h_{i j}(r) \sim-\beta w_{i j}(r)$ for large $r$ we have from eqn (6)

$$
\rho_{i}(r)=\sum_{j} q_{j} n_{j} h_{i j}(r) \sim-\beta \sum_{j} q_{j} n_{j} w_{i j}(r) \sim-\beta \sum_{j} q_{j} n_{j} q_{j}^{\mathrm{eff}} \psi_{i}(r) .
$$

Now, Poisson's equation and eqn (17) imply that for large $r$

$$
\begin{aligned}
\rho_{i}(r) & =-\varepsilon_{\mathrm{r}} \varepsilon_{0} \nabla^{2} \psi_{i}(r) \sim-\frac{\varepsilon_{\mathrm{r}} q_{i}^{\mathrm{eff}}}{4 \pi \mathcal{E}_{\mathrm{r}}^{\mathrm{eff}}}\left[\nabla^{2} \frac{\mathrm{e}^{-\kappa r}}{r}\right] \\
& =-\frac{\varepsilon_{\mathrm{r}} q_{i}^{\mathrm{eff}}}{4 \pi \mathcal{E}_{\mathrm{r}}^{\mathrm{eff}}} \cdot \frac{\kappa^{2} \mathrm{e}^{-\kappa r}}{r} \sim-\varepsilon_{\mathrm{r}} \varepsilon_{0} \kappa^{2} \psi_{i}(r) .
\end{aligned}
$$

** The notation used here differs from that in the original DIT papers ref. 22, 23, 100 and 101 , where the product $\mathcal{E}_{\mathrm{r}}^{\text {eff }} \varepsilon_{0}$ is denoted as $E$ or $\mathcal{E}$ and the present $q_{j}^{\text {eff }}$ is denoted as $q_{j}^{*}$, while $q_{j}^{*}$ in the present work denotes something different (see Section 7).
By comparing the rhs of these two equations we see that the decay parameter $\kappa$ is given by

$$
\kappa^{2}=\frac{\beta}{\varepsilon_{\mathrm{r}} \varepsilon_{0}} \sum_{j} n_{j} q_{j} q_{j}^{\mathrm{eff}}
$$

which is eqn (4). This is an exact equation that has been derived in a strict manner in DIT. ${ }^{100,101}$ When the ion density goes to zero we have $q_{j}^{\text {eff }} \rightarrow q_{j}$ for all $j, \mathcal{E}_{\mathrm{r}}^{\text {eff }} \rightarrow \varepsilon_{\mathrm{r}}$ and $\kappa \rightarrow \kappa_{\mathrm{D}}$.

To conclude, the radial charge density decays like

$$
\rho_{i}(r) \sim-\frac{\kappa^{2} q_{i}^{\text {eff }} \varepsilon_{\mathrm{r}}}{4 \pi \mathcal{E}_{\mathrm{r}}^{\mathrm{eff}}} \cdot \frac{\mathrm{e}^{-\kappa r}}{r} \quad \text { when } r \rightarrow \infty .
$$

The asymptotic expressions (16)-(18) and (20) do not depend on any particular approximation and are valid in general provided that the ion density is not too high. In order to treat higher densities, we must add other contributions to these expressions. This will be done next.

Eqn (18) only describes one decay mode of $w_{i j}(r)$ of the electrolyte system. As mentioned in the Introduction, there exist several decay modes with different decay parameters, $\kappa, \kappa^{\prime}$ etc. and we have $\mathrm{e}^{22,23,106}$ the likewise exact result

$$
w_{i j}(r)=\frac{q_{i}^{\text {eff }} q_{j}^{\text {eff }}}{4 \pi \mathcal{E}_{\mathrm{r}}^{\mathrm{eff}} \varepsilon_{0}} \cdot \frac{\mathrm{e}^{-\kappa r}}{r}+\frac{q_{i}^{\prime} \text { eff } q_{j}^{\prime \text { eff }}}{4 \pi \mathcal{E}_{r}^{\prime} \text { eff } \varepsilon_{0}} \cdot \frac{\mathrm{e}^{-\kappa^{\prime} r}}{r}+\text { other terms }
$$

for $r \geq a$, where $\kappa<\kappa^{\prime}$ for low to moderate ion densities and "other terms" indicate terms from the additional decay modes as well as short-range terms with different decay behaviors. $\dagger \dagger$ Eqn (18) gives only the leading contribution with the longest decay length $1 / \kappa$. Each mode has its own values of the effective charges and the effective permittivity, like $q_{j}^{\text {'eff }}$ and $\mathcal{E}_{\mathrm{r}}^{\prime}$ eff . The decay parameter $\kappa^{\prime}$ is given by

$$
\kappa^{\prime 2}=\frac{\beta}{\varepsilon_{\mathrm{r}} \varepsilon_{0}} \sum_{j} n_{j} q_{j} q_{j}^{\text {eff }}
$$

analogously to eqn (19). The screened Coulomb potential is

$$
\phi_{\text {Coul }}{ }^{*}(r)=\frac{1}{4 \pi \varepsilon_{0}}\left[\frac{\mathrm{e}^{-\kappa r}}{\mathcal{E}_{\mathrm{r}}^{\text {eff }} r}+\frac{\mathrm{e}^{-\kappa^{\prime} r}}{\mathcal{E}_{\mathrm{r}}^{\prime \text { eff }} r}\right]+\text { other terms }
$$

and one may say that this function specifies the decay modes of the electrostatic interactions of an electrolyte.

As an alternative to eqn (19) and (22), one can express both of them as

$$
\kappa^{2}=\frac{\beta}{\varepsilon_{\mathrm{r}} \varepsilon_{0}} \sum_{j} n_{j} q_{j} q_{j}^{\mathrm{eff}}(\kappa)
$$

whereby it is explicit that each mode has its own value of $q_{j}^{\text {eff }}(\kappa)$. In fact, $q_{j}^{\text {eff }}(\kappa)$ can be written as an integral that contains

$\dagger$ Examples of such terms include terms that decay like $f(r) \mathrm{e}^{-2 \kappa r} / r^{2}$, where $f(r)$ is a slowly varying function, ${ }^{23,101}$ and cross-terms between the various modes ( $c f$. ref. 98). Among these terms there are also core-core correlation contributions, which in the restricted primitive model can be distinguished from the electrostatic contributions. In the approximation used in the present work, the core-core contributions are approximated by a hard sphere correlation term $w^{\text {core }}$, see eqn (52). 
distribution functions for the electrolyte. ${ }^{100,101}$ Since $\kappa$ appears on both sides of eqn (24), this formula is an equation for $\kappa$ that has several solutions, $\kappa, \kappa^{\prime}$ etc., that constitute the decay modes. $+\neq$ This fact distinguishes eqn (24) from eqn (1) in a particularly important way. The latter equation gives $\kappa_{\mathrm{D}}$ uniquely and explicitly in terms of the system parameters $q_{j}$, $n_{j}, T$ and $\varepsilon_{\mathrm{r}}$, so there is one single decay mode in the $\mathrm{PB}$ and $\mathrm{DH}$ approximations.

The various decay modes in eqn (21) and (23) give the following principal contributions to the electrostatic potential and charge density for $r \geq a$

$$
\psi_{i}(r)=\frac{q_{i}^{\text {eff }}}{4 \pi \mathcal{E}_{\mathrm{r}}^{\text {eff }} \varepsilon_{0}} \cdot \frac{\mathrm{e}^{-\kappa r}}{r}+\frac{q_{i}^{\text {'eff }}}{4 \pi \mathcal{E}_{\mathrm{r}}^{\prime}{ }^{\prime} \text { fff } \varepsilon_{0}} \cdot \frac{\mathrm{e}^{-\kappa^{\prime} r}}{r}+\text { other terms }
$$

and

$$
\rho_{i}(r)=-\frac{\kappa^{2} q_{i}^{\mathrm{eff}} \varepsilon_{\mathrm{r}}}{4 \pi \mathcal{E}_{\mathrm{r}}^{\mathrm{eff}}} \cdot \frac{\mathrm{e}^{-\kappa r}}{r}-\frac{\kappa^{\prime 2} q_{i}^{\prime} \mathrm{eff}_{\mathrm{r}}}{4 \pi \mathcal{E}_{\mathrm{r}}^{\mathrm{eff}}} \cdot \frac{\mathrm{e}^{-\kappa^{\prime} r}}{r}+\text { other terms }
$$

The first term on the rhs of each equation gives the leading contributions (17) and (20), respectively, for large $r$ when the ion density is sufficiently low.

For elevated ion densities, the leading decay of $w_{i j}(r)$, $\phi_{\text {Coul }^{*}}{ }^{*}(r), \psi_{i}(r)$ and $\rho_{i}(r)$ for large $r$ become exponentially damped oscillatory at a Kirkwood cross-over point. This is included in eqn (21), (23), (25) and (26) and corresponds, as we have seen, to a pair of decay parameters that become complex-valued, $\kappa=\kappa_{\Re}+i \kappa_{\mathfrak{I}}$ and $\kappa^{\prime}=\underline{\kappa}=\kappa_{\Re}-\mathrm{i} \kappa_{\Im}$. This behavior cannot be obtained in the $\mathrm{PB}$ approximation since $\kappa_{\mathrm{D}}$ is a real number. The entities $q_{i}^{\text {eff }}$ and $\mathcal{E}_{\mathrm{r}}^{\text {eff }}$ also become complexvalued at the cross-over point and the corresponding primed quantities become the complex conjugates of the unprimed ones. Beyond the Kirkwood point, the two first terms in eqn (26) yield when $r \rightarrow \infty$

$$
\rho_{i}(r) \sim \frac{|\kappa|^{2}\left|q_{i}^{\mathrm{eff}}\right| \varepsilon_{\mathrm{r}}}{2 \pi\left|\mathcal{E}_{\mathrm{r}}^{\mathrm{eff}}\right|} \cdot \frac{\mathrm{e}^{-\kappa_{\Re} r}}{r} \cos \left(\kappa_{\Im} r+\alpha_{i}\right),
$$

where we have written $\mathcal{E}_{\mathrm{r}}^{\text {eff }}=\left|\mathcal{E}_{\mathrm{r}}^{\text {eff }}\right| \mathrm{e}^{-\mathrm{i} \vartheta}, q_{i}^{\text {eff }}=\left|q_{i}^{\text {eff }}\right| \mathrm{e}^{-i \eta_{i}}$ and $\kappa=|\kappa| \mathrm{e}^{-i \theta}$ with real $\vartheta, \eta_{i}$ and $\theta$ and where we have defined $\alpha_{i}=\eta_{i}+2 \theta-\vartheta$.

One can show ${ }^{23,101}$ that before the Kirkwood crossover point we have $\mathcal{E}_{\mathrm{r}}^{\text {eff }}>0$ and $\mathcal{E}_{\mathrm{r}}^{\text {eff }}<0$ and that $\mathcal{E}_{\mathrm{r}}^{\text {eff }}$ and $\mathcal{E}_{\mathrm{r}}^{\text {eff }}$ go to zero at that point. Thus $\mathcal{E}_{\mathrm{r}}^{\text {eff }}$ goes from $\varepsilon_{\mathrm{r}}$ to 0 when the ion density varies from zero to the Kirkwood point. The sum of the two first terms in the rhs of eqn (26) remains, however, finite at that point since the two infinities from $1 / \mathcal{E}_{\mathrm{r}}^{\text {eff }}$ and $1 / \mathcal{E}_{\mathrm{r}}^{\text {eff }}$ in the respective term have different signs and cancel each other.

In this paper we will explore approximations that express the electrostatic correlations in terms of the decay modes with different $\kappa$ values. We will thereby make the following Ansatz

牛 Eqn (24) can be formulated in several equivalent manners in DIT. One of them is an equation based on the static dielectric function $\tilde{\varepsilon}(k)$, namely $\tilde{\varepsilon}(\mathrm{i} \kappa)=0$, where $\mathrm{i}$ is the imaginary unit. The decay modes are hence given by the zeros of $\tilde{\varepsilon}(k)$ in complex Fourier space, which are poles of the Fourier transforms $\tilde{w}_{i j}(k), \tilde{\psi}_{i}(k)$ and $\tilde{\rho}_{i}(k) .^{23,100,101}$ for the electrostatic part $w_{i j}^{\mathrm{el}}$ of the potential of mean force

$$
w_{i j}^{\mathrm{el}}(r)=\frac{q_{i}^{\mathrm{eff}} q_{j}^{\mathrm{eff}}}{4 \pi \mathcal{E}_{\mathrm{r}}^{\mathrm{eff}} \varepsilon_{0}} \cdot \frac{\mathrm{e}^{-\kappa r}}{r}+\frac{q_{i}^{\prime} \text { eff } q_{j}^{\prime \text { eff }}}{4 \pi \mathcal{E}_{\mathrm{r}}^{\prime} \varepsilon_{0}} \cdot \frac{\mathrm{e}^{-\kappa^{\prime} r}}{r} \quad \text { when } r \geq a,
$$

so the two leading modes in eqn (21) are included (we will also investigate cases where only one mode is included). This means that we assume that these contributions to $w_{i j}^{\text {el }}$ are valid for all $r$ down to contact, $r \geq a$, and we neglect all other terms in $w_{i j}^{\mathrm{el}}$, which decay faster than the leading mode. The main contributions of these other terms appear for small $r$, so we expect deviations from the exact $w_{i j}(r)$ for small $r$ values. Our Ansatz will work for cases where these deviations are sufficiently small. The complete $w_{i j}(r)$ used in the current work also contains a contribution $w_{i j}^{\text {core }}(r)$ from hard-core correlations among the ions. It will be specified in Section 4.2 and is important only for high densities. In the development of the theory we will gradually introduce various levels of refinements, whereby we will start with $\rho_{i}(r)$ given by the first two decay mode terms of

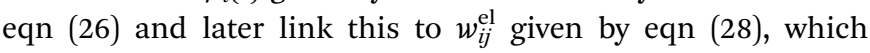
constitutes the basic approximation used in our approach.

Our approximation (28) is similar to the so-called exponential Debye-Hückel (DHX) approximation, ${ }^{71,107}$ where the rhs of eqn (9) is used as an approximation for $w_{i j}(r)$ in $g_{i j}(r)=\mathrm{e}^{-\beta w_{i j}(r)}$, that is,

$$
w_{i j}(r)=\frac{q_{i}^{\text {eff }} q_{j}}{4 \pi \varepsilon_{\mathrm{r}} \varepsilon_{0}} \cdot \frac{\mathrm{e}^{-\kappa_{\mathrm{D}} r}}{r} \quad \text { when } r \geq a \quad(\mathrm{DHX}),
$$

whereby the DH value of $q_{i}^{\text {eff }}$ from eqn (12) is used. The DHX approximation violates several necessary requirements of statistical mechanics for electrolytes, but it has nevertheless some merits. $8,16,71,75,76$

Our Ansatz (28) is accordingly an extension of the DHX approximation to several decay modes. A major difference between it and the DHX approximation is that the effective entities $q_{i}^{\text {eff }}, \mathcal{E}_{\mathrm{r}}^{\text {eff }}$ etc. in eqn (28) can be determined in a selfconsistent manner from requirements that need to be fulfilled. In contrast, $q_{i}^{\text {eff }}$ in the DHX approximation is explicitly determined by eqn (12) from the system parameters like density, temperature etc., which leads to the violations mentioned earlier.

In the main approximation of the current paper, the electrostatic response is linearized. This approximation will be denoted as the Multiple-Decay Extended Debye-Hückel, MDE-DH, approximation and is used in the first part of the current work, before Section 5. The linearization motivates the designation "an extended Debye-Hückel approximation," since the DH approximation is a linear approximation for the electrostatic response. In the last part of the work, nonlinear contributions are studied and an extended DHX approximation is used.

Approximations that explicitly deal with several decay modes have been proposed earlier. Xiao and Song ${ }^{48-50}$ have developed a linear theory for electrolytes that they designate as a molecular Debye-Hückel theory (they use the acronym $\mathrm{MDH}$, which should not be confused with MDH used in the current work). This theory, which is based on DIT, focuses on the decay modes of $\psi_{i}(r)$ as expressed in eqn (25) with several Yukawa function 
terms present, one for each decay mode with decay parameter $\kappa_{l}$. These modes are obtained from the static dielectric function $\tilde{\varepsilon}(k)$, where $k$ is the wave number, via an equation that is equivalent to eqn (24). $\S \S$ They write $\psi_{i}(r)$ in terms of the Yukawa functions of all decay modes, $\psi_{i}(r)=\sum_{l} C_{i, l} \mathrm{e}^{-\kappa_{l} r} / r$ for $r \geq a$, where $l$ in principle runs over all modes, whereby they neglect all other contributions to $\psi_{i}$. In practice the sum is limited to a few modes. The coefficients $C_{i, l}$ are determined from equations that use the dielectric function $\tilde{\varepsilon}(k)$ as input, whereby the latter can be taken from various theories like the MSA, LMPB, MDH or HNC approximations. The results are then used to calculate thermodynamical properties of the system. This approximation is closely related to the simplest version of the MDE-DH approximation of the current work, but it is not identical because the latter contains nonlinear contributions, which are important in the pair distributions and for thermodynamical consistency, as will be seen.

Another approach is that of Outhwaite and Bhuiyan, ${ }^{31}$ who have used the LMPB approximation to calculate $\psi_{i}(r)$ in manner that also shares features with the simplest version of the MDE-DH approximation in the present work. They obtain $\kappa$ and $\kappa^{\prime}$ as the two smallest solutions of the LMPB equation for the decay parameters, whereby $\psi_{i}(r)$ is approximated for $r \geq 2 a$ like in eqn (3) and obtained for $r<2 a$ by other means. The coefficients that correspond to $C$ and $C^{\prime}$ are determined via various alternative conditions on $\psi_{i}$, where one alternative is essentially the same as in the current work.

\section{Modified Debye-Hückel approximations with a single decay-length}

We saw in the Introduction that several approaches with a single decay lengths go quite a long way towards the objective to accurately obtain thermodynamical entities for electrolytes in a simple manner. In order to explicitly see why a single mode nevertheless is not sufficient in general, we will investigate two such approaches that are particularly relevant for the understanding of the approximations suggested in the current work.

As a preparation for the Multiple-Decay Extended $\mathrm{DH}$ approximation, let us consider the Modified Debye-Hückel $(\mathrm{MDH})$ approximation by Kjellander, ${ }^{32}$ where one uses the following Ansatz with a single decay mode

$$
\rho_{i}^{\mathrm{tot}}(r)=\left\{\begin{array}{ll}
q_{i} \delta^{(3)}(r), & r<a \\
-\frac{\kappa^{2} q_{i}^{\mathrm{eff}}}{4 \pi} \cdot \frac{\mathrm{e}^{-\kappa r}}{r}, & r \geq a
\end{array}(\mathrm{MDH}),\right.
$$

which differs from the DH result in eqn (14) solely by the values of the decay parameter $\kappa \neq \kappa_{\mathrm{D}}$ and the effective charge $q_{i}^{\text {eff }}$. This Ansatz means that the leading term in the large $r$ decay formula, as given in eqn (20), is assumed to be valid for all $r$ outside the ion and that $\mathcal{E}_{\mathrm{r}}^{\mathrm{eff}}=\varepsilon_{\mathrm{r}}$, so the value of $\mathcal{E}_{\mathrm{r}}^{\mathrm{eff}}$ for low ion

$\S \S$ See footnote + above. densities is assumed. Like the $\mathrm{DH}$ approximation, $\mathrm{MDH}$ is a linear approximation where the charge density of the ion cloud around an ion is proportional to the charge $q_{i}$. It can therefore be valid only for sufficiently low electrostatic coupling (high temperatures and/or high $\varepsilon_{\mathrm{r}}$ ). By inserting eqn (30) into the condition of local electroneutrality we obtain

$$
q_{i}-\int_{a}^{\infty} \mathrm{d} r 4 \pi r^{2} \frac{\kappa^{2} q_{i}^{\text {eff }} \mathrm{e}^{-\kappa r}}{4 \pi r}=q_{i}-q_{i}^{\text {eff }} \mathrm{e}^{-\kappa a}(1+\kappa a)=0,
$$

which gives

$$
q_{i}^{\mathrm{eff}}=q_{i} \frac{\mathrm{e}^{\kappa a}}{1+\kappa a}
$$

(cf. eqn (12)). The mean electrostatic potential $\psi_{i}(r)$ equals

$$
\psi_{i}(r)=\frac{q_{i}^{\text {eff }}}{4 \pi \varepsilon_{\mathrm{r}} \varepsilon_{0}} \cdot \frac{\mathrm{e}^{-\kappa r}}{r} \quad \text { when } r \geq a \quad(\mathrm{MDH}),
$$

as follows for Poisson's equation.

The deviation of $q_{i}^{\text {eff }}$ from $q_{i}$ expressed by eqn (31) is caused simply by the excluded volume that the ionic core gives rise to in the radial charge distribution. When all ions are treated on the same basis so all have $q_{j}^{\text {eff }} \neq q_{j}$, this deviation has quite far-reaching effects as we now will see. By inserting eqn (31) into the exact eqn (19) and using eqn (1) we obtain the $\mathrm{MDH}$ result ${ }^{32}$

$$
\left[\frac{\kappa}{\kappa_{\mathrm{D}}}\right]^{2}=\frac{\mathrm{e}^{\kappa a}}{1+a \kappa} .
$$

This equation for $\kappa$ has also been obtained in a different manner by $\mathrm{Hall}^{33}$ in his LT approximation and recently by Ding et al. in their the charge renormalization theory. ${ }^{40}$ It can be solved numerically to give $\kappa$ as function of $\kappa_{\mathrm{D}}$ and when this solution inserted into eqn (31), one obtains $q^{\text {eff }}$ as a function of $\kappa_{\mathrm{D}}$. For low ion densities, where $\kappa \approx \kappa_{\mathrm{D}}$, eqn (30) agrees with the Debye-Hückel expression (11) for $\rho_{i}$, but it differs otherwise.

In order to illustrate how the solution to eqn (33) behaves, let us write eqn (33) as $f(\kappa a)=\kappa_{\mathrm{D}} a$, where $f(x)=\left[x^{2}(1+x) / \mathrm{e}^{x}\right]^{1 / 2}$ and plot $f$ as function of $\kappa a$. This function is plotted as the full curve in Fig. 1 and the solution is the intersection point between the curve and a horizontal line at $f=\kappa_{\mathrm{D}} a$; the figure shows an example with $\kappa_{\mathrm{D}} a=0.477$ for which the intersection (full circle) occurs at $\kappa a=0.500$. In the figure we see that there is a second intersection point, which is shown as an open circle. This point also corresponds to a solution of eqn (33), which hence has two solutions for $\kappa$ which we will denote as $\kappa$ and $\kappa^{\prime}$, respectively. These two solutions exists for any $\kappa_{\mathrm{D}} a<1.346$, which is the maximum of the curve; the latter occurs for $\kappa a=1+\sqrt{3} \approx 2.732$ and is marked by a full square in the figure. The function $f(x)$ goes to zero when $x \rightarrow \infty$, so the right-hand intersection occurs for increasingly large $\kappa a$ when $\kappa_{\mathrm{D}} a$ goes to zero, which means that $\kappa^{\prime} \rightarrow \infty$ in this limit. As we will see shortly, this solution is physically relevant and gives rise to a second decay mode.

We have thus shown that eqn (33) has two solutions $\kappa$ and $\kappa^{\prime}$ with $\kappa<\kappa^{\prime}$, where $\kappa$ goes to $\kappa_{\mathrm{D}}$ and $\kappa^{\prime}$ goes to infinity when $\kappa_{\mathrm{D}} a$ 


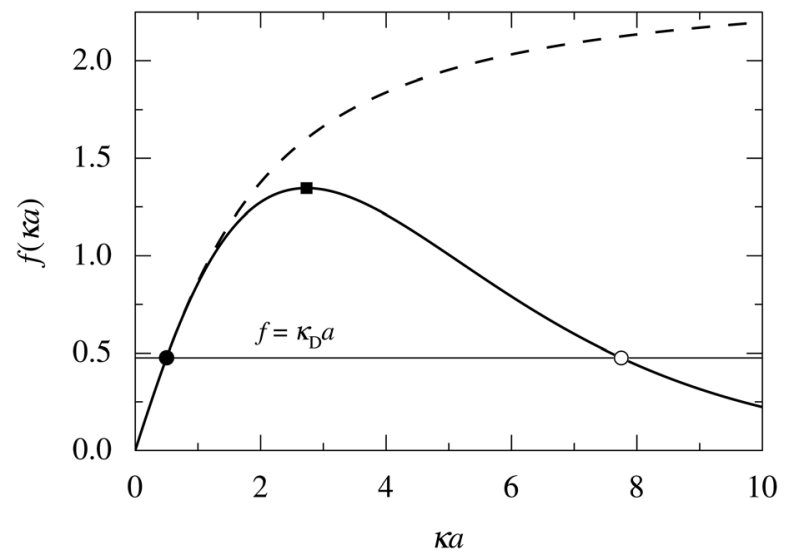

Fig. 1 Plots of $f(\kappa a)$ for two different functions: the full curve shows $f(x)=$ $\left[x^{2}(1+x) / e^{x}\right]^{1 / 2}$ and the dashed curve shows $f(x)=\left[x^{2}(1+x) / \exp _{3}(x)\right]^{1 / 2}$, where $\exp _{3}(x)$ is an exponential sum function defined in eqn (37). The horizontal line shows $f=\kappa_{\mathrm{D}} a$ with $\kappa_{\mathrm{D}} a=0.477$. This line has two intersections with the full curve that are shown as a full circle and an open circle, respectively. The two intersections correspond to the two solutions $\kappa$ and $\kappa^{\prime}$ of eqn (33) when $\kappa_{\mathrm{D}} a=0.477$. The maximum of the full curve is marked with a full square.

goes to zero, or in other words, when the ion density goes to zero. The two solutions $\kappa$ and $\kappa^{\prime}$ appear despite the fact that the MDH theory was set up by using the ansatz in eqn (30) with only one $\kappa$ parameter. This is an inconsistency that we will resolve later. When the ion density and hence $\kappa_{\mathrm{D}} a$ are increased, $\kappa$ and $\kappa^{\prime}$ approach each other and beyond $\kappa_{\mathrm{D}} a=1.35$, i.e., above the maximum in Fig. 1, the solutions $\kappa$ and $\kappa^{\prime}$ to eqn (33) become complex-valued, $\kappa=\kappa_{\Re}+\mathrm{i} \kappa_{\mathfrak{I}}$ and $\kappa^{\prime}=\underline{\kappa}=\kappa_{\Re}-\mathrm{i} \kappa_{\Im}$, which is in agreement with the general result mentioned above in Section 2. 9 ๆ

The values of $\kappa / \kappa_{\mathrm{D}}, \kappa^{\prime} / \kappa_{\mathrm{D}}, \kappa_{\Re} / \kappa_{\mathrm{D}}$ and $\kappa_{\mathfrak{\Im}} / \kappa_{\mathrm{D}}$ from the solutions to eqn (33) are plotted as functions of $\kappa_{\mathrm{D}} a$ in Fig. 2a and are compared with results from Monte Carlo (MC) simulations and Hypernetted Chain (HNC) approximation calculations of the decay of $\psi_{i}(r)$ and $\rho_{i}(r)$ for a 1:1 electrolyte in water at room temperature with $a=4.6 \AA$. These results also correspond to a classical 1:1 plasma in vacuum $\left(\varepsilon_{\mathrm{r}}=1\right)$ when $T=23400 \mathrm{~K}$, which has the same value of $k_{\mathrm{B}} T \varepsilon_{\mathrm{r}}$. The HNC approximation is very accurate for these systems. It is seen in the figure that the predictions from eqn (33) agree very well with the MC and HNC results, including the Kirkwood crossover point and beyond. || || This is quite remarkable considering the humble origin of eqn (33). Obviously, the second root of eqn (33) and the cross-over to oscillatory decay have a great physical relevance. We will explore this in the next section. Fig. $2 \mathrm{~b}$ shows a plot of $\kappa_{\Re} / \kappa_{\mathrm{D}}$ and $\kappa_{\Im} / \kappa_{\mathrm{D}}$ from eqn (33) for larger values of $a \kappa_{\mathrm{D}}$. Other linear approximations like the MSA and LMPB theories, which are considerably more complicated, give predictions that are very similar to those shown in the figure.

Tा There also exist other complex-valued solutions to eqn (33), but here we are only concerned with those two that are connected continuously with the two real solutions $\kappa$ and $\kappa^{\prime}$.

\|\| The HNC data for $\kappa_{\mathrm{D}} a \gtrsim 1.5$ in ref. 22 were not reliable and have been ignored in the plot.
Apart from the local electroneutrality condition that the charge distribution has to fulfill, there is also the Stillinger-Lovett second moment condition ${ }^{108}$ that must also be fulfilled. This condition expresses the fact that from the point of view of electrostatics, electrolytes behave like perfect conductors. It can be formulated as

$$
\beta \sum_{i} q_{i} n_{i} \int \mathrm{d} \mathbf{r} r^{2} \rho_{i}(r)=-6 \varepsilon_{\mathrm{r}} \varepsilon_{0}
$$

which can alternatively be written in a more common manner as an equation involving the second moment of the pair correlation functions $h_{i j}(r)=g_{i j}(r)-1$. This condition is not fulfilled for the MDH approximation [i.e., eqn (30) with $\kappa$ given by eqn (33)], but this approximation does, however, fulfill it approximately for low to medium concentrations and is much better in this respect than the DH approximation. ${ }^{33}$

We may strictly enforce the Stillinger-Lovett condition by refraining from the assumption that $\mathcal{E}_{\mathrm{r}}^{\text {eff }}=\varepsilon_{\mathrm{r}}$ and select the value of $\mathcal{E}_{\mathrm{r}}^{\text {eff }}$ so that eqn (34) is satisfied. Guided by eqn (20) we then take

$$
\rho_{i}(r)=-\frac{\kappa^{2} q_{i}^{\mathrm{eff}} \varepsilon_{\mathrm{r}}}{4 \pi \mathcal{E}_{\mathrm{r}}^{\mathrm{eff}}} \cdot \frac{\mathrm{e}^{-\kappa r}}{r} \quad \text { when } r \geq a
$$

If we insert this into eqn (34) and use the exact eqn (19) we obtain

$$
\frac{\mathcal{E}_{\mathrm{r}}^{\mathrm{eff}}}{\varepsilon_{\mathrm{r}}}=\mathrm{e}^{-\kappa a}\left[1+\kappa a+\frac{(\kappa a)^{2}}{2}+\frac{(\kappa a)^{3}}{6}\right]=\mathrm{e}^{-\kappa a} \exp _{3}(\kappa a),
$$

where we have defined the "exponential sum functions"

$$
\exp _{\ell}(x)=\sum_{\nu=0}^{\ell} \frac{x^{\nu}}{n !}
$$

(note that $\exp _{\infty}(x)=\exp (x)=\mathrm{e}^{x}$ ). Eqn (36) implies that $1 \geq \mathcal{E}_{\mathrm{r}}^{\text {eff }} / \varepsilon_{\mathrm{r}} \gtrsim 0.98$ for $\kappa a<1$, so it is a good approximation to set $\mathcal{E}_{\mathrm{r}}^{\mathrm{eff}}=\varepsilon_{\mathrm{r}}$ for low to medium ion densities as done in the MDH approximation.

By applying the local electroneutrality to eqn (35) we obtain

$$
q_{i}^{\text {eff }}=q_{i} \frac{\mathrm{e}^{\kappa a}}{1+\kappa a} \cdot \frac{\mathcal{E}_{\mathrm{r}}^{\mathrm{eff}}}{\varepsilon_{\mathrm{r}}}=q_{i} \frac{\exp _{3}(\kappa a)}{1+\kappa a},
$$

where we have inserted $\mathcal{E}_{\mathrm{r}}^{\text {eff }} / \varepsilon_{\mathrm{r}}$ from eqn (36) to get the last equality. Using eqn (19), we obtain

$$
\left[\frac{\kappa}{\kappa_{\mathrm{D}}}\right]^{2}=\frac{\exp _{3}(\kappa a)}{1+a \kappa} \quad(\mathrm{SCSL}) .
$$

This equation for $\kappa$ is the Self-Consistent Screening Length (SCSL) approximation by Attard ${ }^{84}$ mentioned earlier, which was set up as an approximation that satisfies both the local electroneutrality and the second moment conditions. In order to investigate $\kappa$ obtained in this approximation, let us this time define $f(x)=\left[x^{2}(1+x) / \exp _{3}(x)\right]^{1 / 2}$ whereby eqn (38) corresponds to $f(\kappa a)=\kappa_{\mathrm{D}} a$. In Fig. 1 the plot of this $f$ as function of $\kappa a$ is shown as the dashed curve and we see that there is only one intersection with the horizontal line $f(\kappa a)=\kappa_{\mathrm{D}} a$. Therefore we 

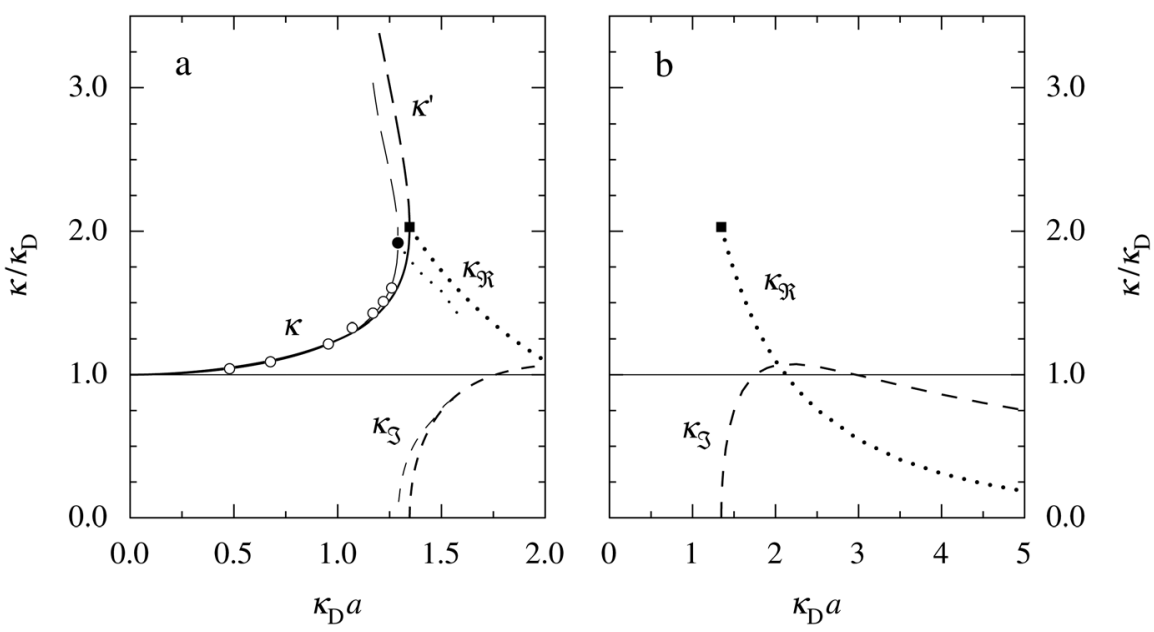

Fig. 2 (a) Decay parameters divided by the Debye parameter $\kappa_{\mathrm{D}}$ for a $1: 1$ aqueous electrolyte solution at room temperature as functions of $\kappa_{\mathrm{D}}$, which is proportional to the square root of the ion density. The same results apply to a classical 1: 1 plasma in vacuum at $T=23400 \mathrm{~K}$. Thick curves: obtained from the solutions of eqn (33); thin curves: HNC results and open symbols: MC simulation results. The Kirkwood crossover point is shown as a filled symbol for the HNC results and those from eqn (33). The MC data are taken from ref. 18 and the HNC data from ref. 21 and 22. (b) The real and imaginary parts of $\kappa$ from eqn (33) divided by $\kappa_{\mathrm{D}}$.

see that this approximation does not predict a Kirkwood crossover point. Instead, the single real solution $\kappa$ goes to infinity when $\kappa_{\mathrm{D}} a \rightarrow 6$, which follows from the fact that $f(x) \rightarrow 6$ when $x \rightarrow \infty$. For small $\kappa_{\mathrm{D}} a$, however, the results of this approximation are accurate since the full and the dashed curves virtually coincide there. When $\kappa_{\mathrm{D}} a>6$, eqn (38) has only complex-valued solutions.

This is a clear example of the fact that one may not achieve an improvement of an approximate theory by enforcing a necessary condition. The problem is that it is too restrictive to assume that there only exist one decay mode $\mathrm{e}^{-\kappa r} / r$ for the charge density, so the enforcement of the Stillinger-Lovett condition leads to a theory that gives qualitatively incorrect results for high ion densities. In order to obtain a Kirkwood crossover point we need two decay modes $\mathrm{e}^{-\kappa r} / r$ and $\mathrm{e}^{-\kappa^{\prime} r} / r$. Thereby the oscillatory decay for high ion densities can be obtained in a correct manner. The MDH and SCSL approximations with a single decay mode can be used only for low to moderate ion densities. We will return to the SCSL approximation in Section 5; here we will continue with $\mathrm{MDH}$ approximation that is more accurate.

The MDH Ansatz (30) corresponds, as we will verify shortly, to the approximations $w_{i j}(r)=q_{j}^{\text {eff }} \psi_{i}(r)$ and $g_{i j}(r)=\mathrm{e}^{-\beta w_{i j}(r)} \approx$ $1-\beta w_{i j}(r)$, so by inserting $\psi_{i}$ from eqn (32) we obtain

$g_{i j}(r)=1-\beta q_{j}^{\text {eff }} \psi_{i}(r)=1-\frac{\beta q_{j}^{\text {eff }} q_{i}^{\text {eff }}}{4 \pi \varepsilon_{r} \varepsilon_{0}} \cdot \frac{\mathrm{e}^{-\kappa r}}{r}$ when $r \geq a$

$(\mathrm{MDH})$

In the DH approximation, where $\psi_{i}$ is given by eqn (13), we have instead

$$
g_{i j}(r)=1-\beta q_{j} \psi_{i}(r)=1-\frac{\beta q_{j} q_{i}^{\text {eff }}}{4 \pi \varepsilon_{\mathrm{r}} \varepsilon_{0}} \cdot \frac{\mathrm{e}^{-\kappa_{\mathrm{D}} r}}{r} \text { when } r \geq a
$$

Note that $h_{i j}(r)=g_{i j}(r)-1$ is proportional to $q_{i} q_{j}$ in both cases since the effective charge is proportional to the actual (bare) change in these approximations.

The $g_{i j}(r)$ in eqn (39) gives the charge density in eqn (30), as can be easily verified as follows. We have

$$
\begin{aligned}
\rho_{i}(r) & =\sum_{j} q_{j} n_{j} g_{i j}(r)=-\sum_{j} \frac{\beta q_{j} n_{j} q_{j}^{\text {eff }}}{\varepsilon_{\mathrm{r}} \varepsilon_{0}} \cdot \frac{q_{i}^{\text {eff }}}{4 \pi} \cdot \frac{\mathrm{e}^{-\kappa r}}{r} \\
& =-\frac{\kappa^{2} q_{i}^{\text {eff }}}{4 \pi} \cdot \frac{\mathrm{e}^{-\kappa r}}{r} \quad \text { when } r \geq a \quad(\mathrm{MDH}),
\end{aligned}
$$

where we have used the exact eqn (19) for $\kappa$ and the fact that $\sum_{j} n_{j} q_{j}=0$. This is the same as the second line in eqn (30). In the DH approximation we instead obtain the charge density given by eqn (14) for $r \geq a$

$$
\rho_{i}(r)=-\sum_{j} \frac{\beta n_{j} q_{j} q_{j}}{\varepsilon_{\mathrm{r}} \varepsilon_{0}} \cdot \frac{q_{i}^{\text {eff }}}{4 \pi} \cdot \frac{\mathrm{e}^{-\kappa_{\mathrm{D}} r}}{r}=-\frac{\kappa_{\mathrm{D}}^{2} q_{i}^{\text {eff }}}{4 \pi} \cdot \frac{\mathrm{e}^{-\kappa_{\mathrm{D}} r}}{r}(\mathrm{DH}),
$$

where we have used the definition of $\kappa_{\mathrm{D}}$ in eqn (1). We can see that the appearance of a decay parameter $\kappa \neq \kappa_{\mathrm{D}}$ in the MDH approximation is intimately linked to the treatment of all ions on the same basis, so all ions of species $j$ have an effective charge $q_{j}^{\text {eff }} \neq q_{j}$. As pointed our earlier, in the nonlinear Poisson-Boltzmann and the DH approximations, the central ion with effective charge $q_{i}^{\text {eff }} \neq q_{i}$ is treated differently from the ions in its surroundings. The latter are treated as point ions that do not correlate with each other, so for them we can say that $q_{j}^{\text {eff }}=q_{j}$ and by inserting this into eqn (19) we obtain the DH expression (1) for $\kappa_{\mathrm{D}}$.

The total ion density around an $i$-ion is equal to $\sum_{j} n_{j} g_{i j}(r)$. In the $\mathrm{DH}$ and $\mathrm{MDH}$ approximations, this density is equal to the bulk value $n_{\text {tot }}$ for $r \geq a$ because it follows from eqn (39) and (40) that $\sum_{j} n_{j} g_{i j}(r)=n_{\text {tot }}$. 


\section{Debye-Hückel extensions with multiple decay lengths}

\subsection{Decay parameters and effective permittivities}

To resolve the inconsistencies of the single decay mode approaches, we use eqn (26) as basis for a better approximation. We take the first two terms as an Ansatz that we assume holds for all $r \geq a$

$$
\rho_{i}^{\text {tot }}(r)= \begin{cases}q_{i} \delta^{(3)}(r), & r<a \\ -\frac{\varepsilon_{\mathrm{r}}}{4 \pi}\left[\frac{\kappa^{2} q_{i}^{\text {eff }}}{\mathcal{E}_{\mathrm{r}}^{\text {eff }}} \cdot \frac{\mathrm{e}^{-\kappa r}}{r}+\frac{\kappa^{\prime 2} q_{i}^{\text {eff }}}{\mathcal{E}_{r}^{\prime \text { eff }}} \cdot \frac{\mathrm{e}^{-\kappa^{\prime} r}}{r}\right], & r \geq a .\end{cases}
$$

where the second line is equal to $\rho_{i}(r)$ for $r \geq a$. We will, however, keep eqn (31) and hence eqn (33) as they are because the latter gives good results for $\kappa$ and $\kappa^{\prime}$, including the Kirkwood crossover, for monovalent ions in water at room temperature and for the classical plasma at high temperatures. These equations are also fulfilled by the primed quantities and the decay parameters used are those plotted in Fig. 2. Recall that the behavior of the decay modes as seen in this figure was obtained from an effect of excluded volume in the radial ion distribution, as expressed in terms of an effective charge in eqn (31) for each mode.

The Ansatz in eqn (43) constitutes an important ingredient of the simplest version of the MDE-DH approximation, which we will denote as the Simple $M D E-D H$ approximation. We will later formulate the Complete $M D E-D H$ approximation [see eqn (54), (55) and (97)], but eqn (43) is sufficient in many cases and works very well provided that the ion size is not too large and core-core correlations are not too prominent.

By applying the local electroneutrality $\int \mathrm{d} \mathbf{r} \rho_{i}^{\text {tot }}(r)=0$ to eqn (43) and using eqn (31) and its analogue for the primed quantities, we can readily deduce that

$$
\frac{\varepsilon_{\mathrm{r}}}{\mathcal{E}_{\mathrm{r}}^{\mathrm{eff}}}+\frac{\varepsilon_{\mathrm{r}}}{\mathcal{E}_{\mathrm{r}}^{\text {eff }}}=1
$$

To determine $\mathcal{E}_{\mathrm{r}}^{\text {eff }}$ and $\mathcal{E}_{\mathrm{r}}^{\text {eff }}$ we need one more equation, which we can obtain by applying the Stillinger-Lovett condition to the ansatz in eqn (43) and use the exact eqn (19) and (22). This yields

$$
\frac{\varepsilon_{\mathrm{r}}}{\mathcal{E}_{\mathrm{r}}^{\text {eff }}} \mathrm{e}^{-\kappa a} \exp _{3}(\kappa a)+\frac{\varepsilon_{\mathrm{r}}}{\mathcal{E}_{\mathrm{r}}^{\text {eff }}} \mathrm{e}^{-\kappa^{\prime} a} \exp _{3}\left(\kappa^{\prime} a\right)=1 .
$$

Together, these two equations for $\mathcal{E}_{\mathrm{r}}^{\text {eff }}$ and $\mathcal{E}_{\mathrm{r}}^{\text {eff }}$ imply that

$$
\begin{aligned}
\frac{\mathcal{E}_{\mathrm{r}}^{\mathrm{eff}}}{\varepsilon_{\mathrm{r}}} & =\frac{\mathrm{e}^{-\kappa a} \exp _{3}(\kappa a)-\mathrm{e}^{-\kappa^{\prime} a} \exp _{3}\left(\kappa^{\prime} a\right)}{1-\mathrm{e}^{-\kappa^{\prime} a} \exp _{3}\left(\kappa^{\prime} a\right)} \\
\frac{\mathcal{E}_{\mathrm{r}}^{\text {eff }}}{\varepsilon_{\mathrm{r}}} & =-\frac{\mathrm{e}^{-\kappa a} \exp _{3}(\kappa a)-\mathrm{e}^{-\kappa^{\prime} a} \exp _{3}\left(\kappa^{\prime} a\right)}{1-\mathrm{e}^{-\kappa a} \exp _{3}(\kappa a)} .
\end{aligned}
$$

One can show that these relationships imply that $\varepsilon_{\mathrm{r}} \geq \mathcal{E}_{\mathrm{r}}^{\text {eff }} \geq 0$ and $-\infty \leq \mathcal{E}_{r}^{\prime \text { eff }} \leq 0$, so $\mathcal{E}_{\mathrm{r}}^{\prime \text { eff }}$ is negative in agreement with the general results mentioned in Section 2 . The value zero occurs at the Kirkwood point, that is,

$$
\kappa=\kappa^{\prime} \quad \Rightarrow \quad \mathcal{E}_{\mathrm{r}}^{\mathrm{eff}}=0, \quad \mathcal{E}_{\mathrm{r}}^{\text {eff }}=0
$$
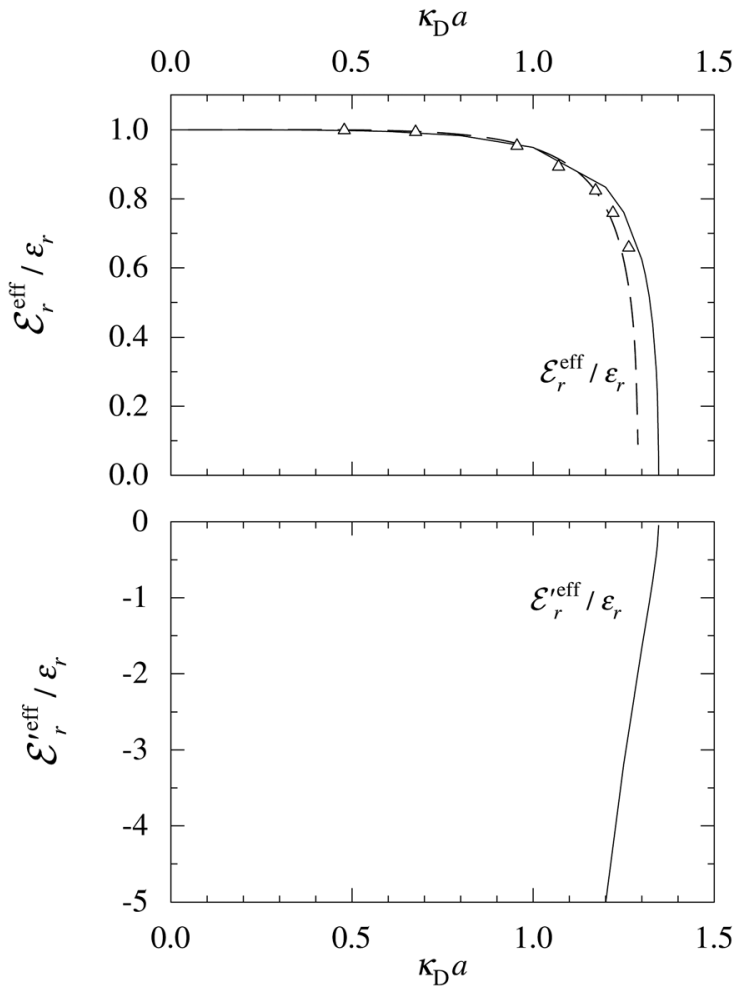

Fig. 3 The ratios $\mathcal{E}_{\mathrm{r}}^{\text {eff }} / \varepsilon_{\mathrm{r}}$ (top frame) and $\mathcal{E}_{\mathrm{r}}^{\prime}$ eff $/ \varepsilon_{\mathrm{r}}$ (bottom frame) as functions of $\kappa_{D} a$. The full curves show the result from eqn (46), the dashed curve show HNC results and the symbols show MC simulation results. The $\mathrm{HNC}$ and MC data are taken from ref. 18 for the same system as in Fig. 2 (see ref. 21 and 22 for further HNC data). Note the difference in ordinate scales of the two frames.

as follows from eqn (46) and (47). This is also in agreement with the general exact results mentioned in Section 2. In the limit of infinite dilution where $\mathcal{E}_{\mathrm{r}}^{\text {eff }} \rightarrow \varepsilon_{\mathrm{r}}$ and $\mathcal{E}_{r}^{\prime \text { eff }} \rightarrow-\infty$, the second term in eqn (43) vanishes and eqn (30) becomes valid, but close to the Kirkwood cross-over point this second term is about equally important as the first term.

In the top frame of Fig. 3 the values of $\mathcal{E}_{\mathrm{r}}^{\mathrm{eff}} / \varepsilon_{\mathrm{r}}$ from eqn (46) are compared to results from MC simulations and the HNC approximation for the same system as in Fig. 2. The agreement is good; the curve from the present theory is somewhat shifted horizontally relative to that from HNC due the the slight difference in Kirkwood point as seen in Fig. 2. The prediction for $\mathcal{E}_{\mathrm{r}}^{\prime}$ eff $/ \varepsilon_{\mathrm{r}}$ is plotted in the bottom frame of Fig. 3 .

For the case where $\kappa$ is complex-valued, $\kappa=\kappa_{\Re}+\mathrm{i} \kappa_{\mathfrak{I}}$, we write $\mathcal{E}_{\mathrm{r}}^{\text {eff }}=\left|\mathcal{E}_{\mathrm{r}}^{\text {eff }}\right| \mathrm{e}^{-\mathrm{i} \vartheta}$ and $\mathcal{E}_{r}^{\prime \text { eff }}=\underline{\mathcal{E}_{\mathrm{r}}^{\text {eff }}}=\left|\mathcal{E}_{\mathrm{r}}^{\text {eff }}\right| \mathrm{e}^{\mathrm{i} \vartheta}$. One then obtains from eqn (44) that

$$
\frac{\left|\mathcal{E}_{\mathrm{r}}^{\mathrm{eff}}\right|}{\varepsilon_{\mathrm{r}}}=2 \cos \vartheta
$$

and one can derive from eqn (46) that

$$
\tan \vartheta=\frac{\mathrm{e}^{\kappa_{\Re} a}-A \cos \left(\kappa_{\Im} a\right)-B \sin \left(\kappa_{\Im} a\right)}{A \sin \left(\kappa_{\Im} a\right)-B \cos \left(\kappa_{\Im} a\right)},
$$




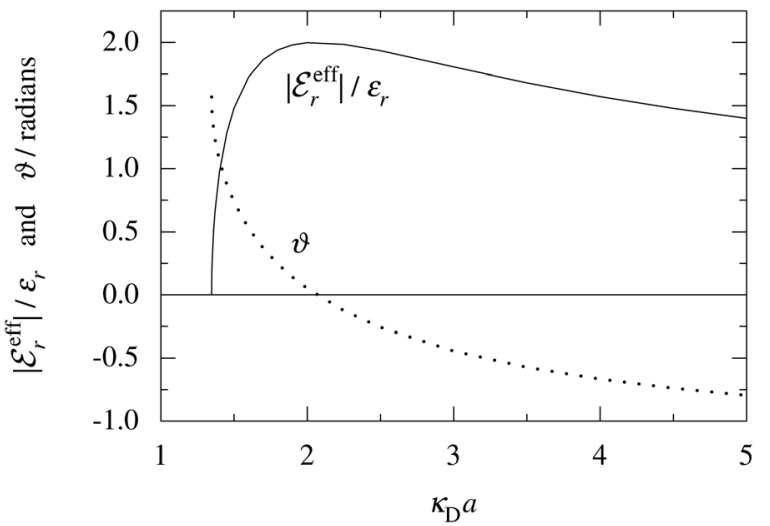

Fig. 4 The ratio $\left|\mathcal{E}_{\mathrm{r}}^{\text {eff }}\right| / \varepsilon_{\mathrm{r}}$ and the phase angle $\vartheta$ of $\mathcal{E}_{\mathrm{r}}^{\text {eff }}=\left|\mathcal{E}_{\mathrm{r}}^{\text {eff }}\right| \mathrm{e}^{-\mathrm{i} \vartheta}$ from eqn (48) and (49) as functions of $\kappa_{\mathrm{D}} a$ beyond the Kirkwood crossover point where $\kappa$ is complex-valued.

where

$$
\begin{gathered}
A=A\left(\kappa_{\Re}, \kappa_{\Im}\right)=\exp _{3}\left(\kappa_{\Re} a\right)-\left(\kappa_{\Im} a\right)^{2} \frac{1+\kappa_{\Re} a}{2} \\
B=B\left(\kappa_{\Re}, \kappa_{\Im}\right)=\kappa_{\Im} a\left[\exp _{2}\left(\kappa_{\Re} a\right)-\frac{\left(\kappa_{\Im} a\right)^{2}}{6}\right] .
\end{gathered}
$$

In Fig. 4 we have plotted $\left|\mathcal{E}_{\mathrm{r}}^{\mathrm{eff}}\right| / \varepsilon_{\mathrm{r}}$ and $\vartheta$ as functions of $\kappa_{\mathrm{D}} a$. At the Kirkwood crossover point, to the left in the figure, we have $\left|\mathcal{E}_{\mathrm{r}}^{\mathrm{eff}}\right|=0$ and $\vartheta=\pi / 2$.

For complex-valued $\kappa$ and $\kappa^{\prime}$ the radial charge distribution is oscillatory and the second line of eqn (43) can be written as (cf. eqn (27))

$$
\rho_{i}(r)=\frac{|\kappa|^{2}\left|q_{i}^{\text {eff }}\right| \varepsilon_{\mathrm{r}}}{2 \pi\left|\mathcal{E}_{\mathrm{r}}^{\text {eff }}\right|} \cdot \frac{\mathrm{e}^{-\kappa_{\Re} r}}{r} \cos \left(\kappa_{\Im} r+\alpha_{i}\right) \quad \text { when } r \geq a,
$$

where $\alpha_{i}=\eta_{i}+2 \theta-\vartheta$ with phases defined from $\mathcal{E}_{\mathrm{r}}^{\text {eff }}=\left|\mathcal{E}_{\mathrm{r}}^{\text {eff }}\right| \mathrm{e}^{-\mathrm{i} \vartheta}$, $q_{i}^{\text {eff }}=\left|q_{i}^{\text {eff }}\right| \mathrm{e}^{-i \eta_{i}}$ and $\kappa=|\kappa| \mathrm{e}^{-i \theta}$ as before. Note that $\theta=$ $-\arctan \left(\kappa_{\mathfrak{s}} / \kappa_{\Re}\right)$.

\subsection{Radial distribution functions}

For binary symmetric electrolytes, the number densities of cations and anions are equal, $n_{+}=n_{-} \equiv n$, the ionic charges are $q_{+}=-q_{-} \equiv q$, the absolute valency $z=q / q_{\mathrm{e}}$ and $n_{\mathrm{tot}}=2 n$. For ions of equal diameter (the restricted primitive model, RPM) we also have $\rho_{+}(r)=-\rho_{-}(r) \equiv \rho(r)$ and $q_{+}^{\text {eff }}=-q_{-}^{\text {eff }} \equiv q^{\text {eff }}$. From eqn (1) and (19) follow

$$
\frac{q^{\mathrm{eff}}}{q}=\left[\frac{\kappa}{\kappa_{\mathrm{D}}}\right]^{2}
$$

which is exact for the RPM.

In the RPM, the electrolyte bulk phase is completely specified by the two dimensionless parameters $\beta_{R} \equiv \beta q^{2} /\left(4 \pi \varepsilon_{\mathrm{r}} \varepsilon_{0} a\right)=$ $z^{2} \ell_{\mathrm{B}} / a$ and $\tau=\kappa_{\mathrm{D}} a$; the latter is the abscissa of Fig. 2-4. Alternatively, any two independent combination of these two parameters can be used to specify an RPM system, like reduced total ionic density $n_{\mathrm{tot} R} \equiv n_{\mathrm{tot}} a^{3}=\tau^{2} /\left(4 \pi \beta_{R}\right)$ and the product $\beta_{R} \tau$. The inverse of the latter is the so-called plasma parameter $4 \pi n_{\mathrm{tot}} / \kappa_{\mathrm{D}}{ }^{3}$.

We will test the predictions of the Simple MDE-DH Ansatz in eqn (43) by comparing with results from MC simulations and HNC calculations for an electrolyte with $\beta_{R}=1.55$, which can be, for example, a 1:1 aqueous electrolyte solution at room temperature with ions of diameter $a=4.6 \AA$ or a classical plasma of such ions in vacuum at $T=23400 \mathrm{~K}$. Depending on the ion density (concentration), the parameter $\tau$ has different values. The radial charge distribution $\rho(r)$ is calculated using $\kappa$ and $\kappa^{\prime}$ obtained from the solutions of eqn (33), $\mathcal{E}_{\mathrm{r}}^{\text {eff }}$ obtained from eqn (46) and $\mathcal{E}_{\mathrm{r}}^{\text {eff }}$ from eqn (47). In Fig. 5 the function
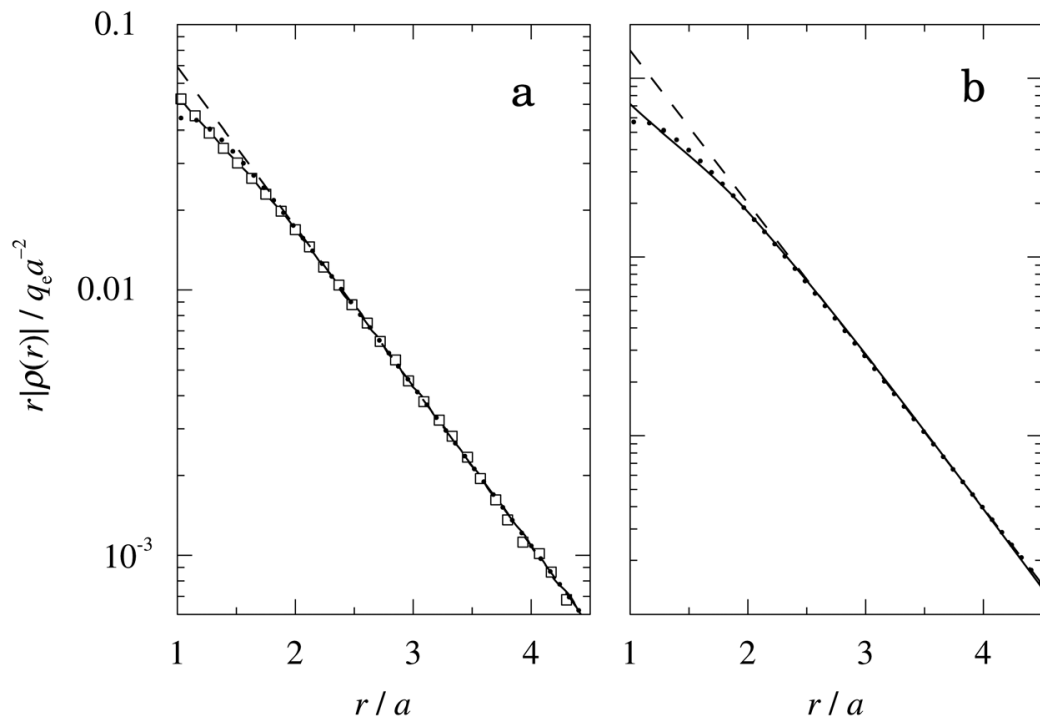

0.1

0.01

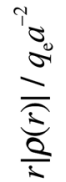

$10^{-3}$

$10^{-4}$

Fig. 5 The radial charge distribution $\rho(r)$ for a 1:1 electrolyte plotted as $r \rho(r)$ as a function of $r$ on a semilogarithmic scale. The ion diameter is $a=4.6 \AA$ and the concentration is $0.5 \mathrm{M}$ in frame (a) and $0.7 \mathrm{M}$ in frame (b) $\left[\tau=\kappa_{\mathrm{D}} a=1.07\right.$ and 1.27 , respectively, and $\left.\beta_{R}=1.55\right]$. The open squares in frame (a) show results from $M C$ simulations ${ }^{18}$ and in both frames the full curves show HNC results, ${ }^{18,21,22}$ the dotted curves show results from the Simple $M D E-D H$ approximation, eqn (43), and the dashed curves show results from solely the first (leading) term in this equation. The unit for $r \rho(r)$ is $q_{\mathrm{e}} a^{-2}$. 
$r|\rho(r)|$ as function of $r$ for the cases of 0.5 and $0.7 \mathrm{M}$ electrolytes is plotted on a semilogarithmic scale, whereby a Yukawa function decay becomes a straight line.

In Fig. 5a we see that the $\mathrm{MC}$ and $\mathrm{HNC}$ results virtually coincide with each other, so the HNC approximation is very accurate for the monovalent electrolyte. The prediction from eqn (43) (dotted curve) agrees very well with the MC and HNC results for all $r$ except in a very short interval just outside the core region, $1 \leq r \lesssim 1.3 a$ and the curves virtually coincide for $r \gtrsim 2 a$. In Fig. $5 \mathrm{~b}$ the agreement is equally good. The dashed curves are calculated from the first term in eqn (43), which is leading for large $r$. In Fig. 5b this curve coincides with the accurate results $r \gtrsim 2.5 a$, but it deviates substantially from the dotted curve for smaller $r$ values. This implies that the contribution from the second Yukawa term with decay parameter $\kappa^{\prime}$ is important for the $0.7 \mathrm{M}$ case. For the $0.5 \mathrm{M}$ it is less important, but it still gives a noticeable contribution that makes the dotted curve to deviate from the dashed one. The difference in importance of the second term for the 0.7 and $0.5 \mathrm{M}$ cases is due to the fact that the magnitude of $\mathcal{E}_{\mathrm{r}}^{\prime}$ eff in the denominator of this term increases rapidly when the concentration is decreased, see Fig. 3. We can conclude that the Ansatz (43) gives very good results for the 1:1 electrolyte systems; the deviation for small $r$ has little consequence in most cases and is caused by terms in $\rho(r)$ with shorter decay lengths that are not included in the Ansatz.

This conclusion is supported by Fig. 6, which shows results for $0.1,0.7$ and $1.0 \mathrm{M}$ electrolytes (the data for $0.7 \mathrm{M}$ are the same as in Fig. 5b). Here, $\rho(r)$ is plotted on a linear scale as $4 \pi r^{2} \rho(r)$, which is proportional to the amount of charge at distance $r$. The curves for $0.1 \mathrm{M}$ show that the first term in eqn (43) dominates for nearly all distances; the second term is very small because $\mathcal{E}_{\mathrm{r}}^{\text {eff }}$ has very large magnitude when the concentration is low. The deviation from the HNC curve occurs, like for the other cases, mainly very close to the core region and has little consequence.

For the $0.7 \mathrm{M}$ case in Fig. 6a, we can very clearly see the importance of the second Yukawa term, since without it, the deviation from the accurate HNC curve is large for $r<2 a$ as apparent from the dashed black curve. Thus it is very important to have the two decay modes present in the theory in order to describe the electrolyte in an appropriate manner. This conclusion is, of course, further emphasized when $\kappa$ and $\kappa^{\prime}$ and become complex-valued at the Kirkwood cross-over point and give rise to oscillations at higher concentrations. The conditions for the $1.0 \mathrm{M}$ case in Fig. 6 are beyond this point, so $\rho(r)$ has an exponentially damped oscillatory decay. We then have complex $\kappa=\underline{\kappa^{\prime}}$ and the two terms in eqn (43) yield the expression for $\rho$ in eqn (50). It is seen from the results from this equation, shown as the red dotted curve in the figure, that the agreement with the accurate HNC curve is about equally good as the results for the other concentrations. Note that the two red curves cross the abscissa axis at virtually the same point in Fig. 6b, which means that the phase $\alpha$ obtained from the Ansatz is very good.

Let us now turn to the pair distribution function $g_{i j}(r)=$ $\mathrm{e}^{-\beta w_{i j}(r)}$. The Ansatz (43) can be obtained from eqn (28) for the

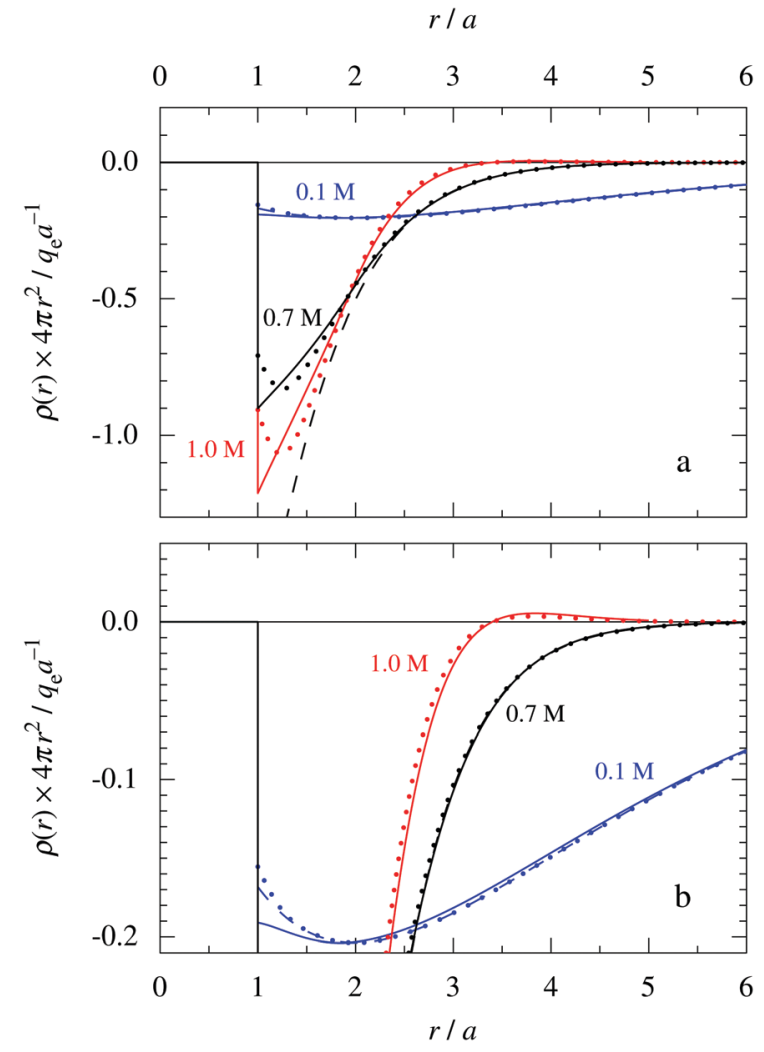

Fig. 6 The radial charge density $\rho(r)$ for a $1: 1$ electrolyte plotted as $4 \pi r^{2} \rho(r)$ as function of $r$. The ion diameter is $a=4.6 \AA$ and the concentration is $0.1 \mathrm{M}$ (red curves), $0.7 \mathrm{M}$ (black curves) and $1.0 \mathrm{M}$ (blue curves) $\left[\tau=\kappa_{\mathrm{D}} \mathrm{a}=0.48,1.27\right.$ and 1.51, respectively, and $\left.\beta_{R}=1.55\right]$. For the cases $0.7 \mathrm{M}$ (same as in Fig. $5 \mathrm{~b}$ ) and $0.1 \mathrm{M}$, the full curves show HNC results, the dotted curves show results from the Simple MDE-DH approximation, eqn (43), and the dashed curves show results from the first term in this equation. In the case of $1.0 \mathrm{M}$, the full curve shows the HNC data and the dotted curve show the results from eqn (50). The bottom frame shows a magnified view of the top one. The HNC data are from ref. 21 and 22 .

electrostatic part, $w_{i j}^{\mathrm{el}}$, of the potential of mean force. When $\beta w_{i j}(r)$ is sufficiently small we can linearize the pair distribution function $g_{i j}(r) \approx 1-\beta w_{i j}(r)$ and when the electrostatic contributions to $w_{i j}$ dominate we can set $w_{i j}(r) \approx w_{i j}^{\text {el }}(r)$, so we have $\rho_{i}(r)=\sum_{j} q_{j} n_{j} g_{i j}(r) \approx \sum_{j} q_{j} n_{j} w_{i j}^{\mathrm{el}}(r)$. By inserting eqn (28) and using eqn (19), we obtain eqn (43) [compare with the derivation of eqn (41)]. As we will see shortly, in the RPM the Ansatz (43) is valid also for somewhat more general conditions.

When the ion density is high, the approximation $w_{i j}(r) \approx$ $w_{i j}^{\text {el }}(r)$ is insufficient because there are important steric contributions missing due to core-core correlations. The latter are in general coupled to the electrostatic correlations, so for simplicity, we will restrict ourselves to symmetric electrolytes in the RPM, where the density-charge correlation function is identically equal to zero and the coupling between electrostatic and core correlations is weak. We make the following Ansatz

$$
g_{i j}(r)=\mathrm{e}^{-\beta\left[w_{i j}^{\mathrm{el}}(r)+w_{i j}^{\mathrm{core}}(r)\right]}
$$


and we approximate $w_{i j}^{\text {core }}(r)$ with the potential of mean force obtained from a hard sphere fluid with pair distribution function $g^{\text {hs }}(r)=\mathrm{e}^{-\beta w \mathrm{hs}(r)}$. The density of the hard sphere fluid is set equal to the total ionic density $n_{\text {tot }}$, that is, the hard sphere fluid has a reduced density $n_{\text {tot }} a^{3}$. A suitable choice of $g^{\text {hs }}(r)$ in numerical calculations is to use the very accurate Verlet-Weis' semi-empirical expression ${ }^{109}$ for $g^{\text {hs }}(r)$. A Fortran code for this expression is publicly available. ${ }^{110}$ The $g^{\text {hs }}(r)$ thus obtained gives contact values $g^{\text {hs }}(a)$ in agreement with the very accurate Carnahan-Starling (CS) equation of state. ${ }^{97}$ The contribution $w_{i j}^{\text {core }}(r)$ expresses a further decay mode for the interactions.

Eqn (52) together with the two-mode Ansatz for $w_{i j}^{\mathrm{el}}(r)$ in eqn (28) constitute a starting point for the formal development of the approximations used in the current work. The details are given in Appendix A. Here we will only give the main points.

In the RPM, the Ansatz (52) implies that

$$
g_{+ \pm}(r)=\mathrm{e}^{-\beta\left[ \pm w^{\mathrm{el}}(r)+w^{\text {core }}(r)\right]}=\mathrm{e}^{\mp \beta w^{\mathrm{el}}(r)} g^{\text {core }}(r)
$$

with $g^{\text {core }}(r)=g^{\text {hs }}(r)$ and we have for $r \geq a$

$$
\begin{aligned}
w^{\mathrm{el}}(r) & =\frac{\left(q^{\mathrm{eff}}\right)^{2}}{4 \pi \mathcal{E}_{\mathrm{r}}^{\mathrm{eff}} \varepsilon_{0}} \cdot \frac{\mathrm{e}^{-\kappa r}}{r}+\frac{\left(q^{\prime} \mathrm{eff}\right)^{2}}{4 \pi \mathcal{E}_{\mathrm{r}}^{\text {eff }} \varepsilon_{0}} \cdot \frac{\mathrm{e}^{-\kappa^{\prime} r}}{r} \\
& =\frac{q^{2}}{4 \pi \varepsilon_{0} \kappa_{\mathrm{D}}{ }^{4}}\left[\frac{\kappa^{4}}{\mathcal{E}_{\mathrm{r}}^{\mathrm{eff}}} \cdot \frac{\mathrm{e}^{-\kappa r}}{r}+\frac{\kappa^{\prime 4}}{\mathcal{E}_{\mathrm{r}}^{\prime} \text { eff }} \cdot \frac{\mathrm{e}^{-\kappa^{\prime} r}}{r}\right],
\end{aligned}
$$

where we have used eqn (51) to obtain the second line.

In the MDE-DH approximations we assume that $\beta w^{\mathrm{el}}(r)$ is sufficiently small so that we can approximate $\mathrm{e}^{\mp \beta w \mathrm{el}(r)} \approx$ $1 \mp \beta w^{\mathrm{el}}(r)+\left[\beta w^{\mathrm{el}}(r)\right]^{2} / 2$. We obtain

$$
g_{+ \pm}(r)=\left[1 \mp \beta w^{\mathrm{el}}(r)+\frac{\left[\beta w^{\mathrm{el}}(r)\right]^{2}}{2}\right]\left[1+h^{\mathrm{core}}(r)\right],
$$

which can be written as

$$
\begin{aligned}
g_{+ \pm}(r)=1 \mp \beta w^{\mathrm{el}}(r)+h^{\mathrm{core}}(r)+\frac{1}{2}\left[\beta w^{\mathrm{el}}(r)\right]^{2} \\
+h^{\mathrm{core}}(r)\left[\frac{1}{2}\left[\beta w^{\mathrm{el}}(r)\right]^{2} \mp \beta w^{\mathrm{el}}(r)\right],
\end{aligned}
$$

where $h^{\text {core }}(r)=g^{\text {core }}(r)-1$. The inclusion of the square term $\left[\beta w^{\mathrm{el}}(r)\right]^{2} / 2$ makes the pair distribution to fulfill the necessary condition $g_{+ \pm}(r) \geq 0$, which can be violated in linear theories like MSA and the DH approximation. Eqn (54) and (55) constitute the Complete MDE-DH approximation for the RPM together with the eqn (31) for $\kappa$, which can be written

$$
\frac{q^{\mathrm{eff}}}{q}=\left[\frac{\kappa}{\kappa_{\mathrm{D}}}\right]^{2}=\frac{\mathrm{e}^{\kappa a}}{1+a \kappa},
$$

and the corresponding relationship for the primed quantities.

The inclusion of a square term $\left[\beta w_{i j}^{\mathrm{el}}(r)\right]^{2} / 2$ from electrostatics has been done for a long time in some kinds of theories, for example as an improvement of the $\mathrm{DH}$ approximation with $w_{i j}^{\text {el }}(r)$ given by eqn (29). ${ }^{10}$ In the present case, the use of the square term of $w_{i j}^{\text {el }}(r)$ with two decay modes and with coefficients that makes $g_{i j}(r)$ satisfy the required electroneutrality and second moment conditions gives, as we will see in the next section, thermodynamic consistency to a considerable degree. In, for example, the GMSA approximation ${ }^{24,47}$ one instead includes an empirical contribution with parameters that are selected to give thermodynamic consistency. The present approach does not contain any such parameters.

In systems where the core-core correlations are not very strong, it is a good approximation to neglect the cross-terms between $h^{\text {core }}$ and $w^{\text {el }}$ in eqn (56) since they are of higher order and are smaller than the other terms for most separations $r$ in such cases. We then obtain for $r \geq a$

$$
g_{+ \pm}(r)=1 \mp \beta w^{\mathrm{el}}(r)+h^{\mathrm{core}}(r)+\frac{1}{2}\left[\beta w^{\mathrm{el}}(r)\right]^{2} .
$$

Together with eqn (54) and (57), this equation constitutes the Simple MDE-DH approximation for the RPM. As shown in Appendix A, this is the approximation behind the Ansatz (43) in the RPM because eqn (58) yields $\rho(r)$ used in the Ansatz (note that $\rho(r)=q n\left[g_{++}(r)-g_{+-}(r)\right]$ in the RPM). The square term and $h^{\text {core }}$ do not contribute to $\rho$ and therefore not to $\psi$. In this approximation all results in Section 4.1 are valid, in particular the expressions for $\mathcal{E}_{\mathrm{r}}^{\text {eff }}$ and $\mathcal{E}_{\mathrm{r}}^{\text {'eff }}$ in eqn (44)-(49). The corresponding expressions in the Complete MDE-DH approximation are given in Appendices A and B.

Since we have retained solely the linear and quadratic terms in $\beta w^{\text {el }}$ in both MDE-DH approximations, their range of validity is clearly limited to systems at sufficiently high temperatures and/or high $\varepsilon_{\mathrm{r}}$ so that $\beta_{R}$ is sufficiently low. For monovalent ions of diameter $4.6 \AA$ in aqueous solution at room temperature, which constitute the system for the $\mathrm{MC}$ simulations that we successfully have compared with in the previous examples, we have $\beta_{R}=1.55$. The appropriate $\beta_{R}$ values for the MDE-DH approximations apparently are of this order of magnitude or less.

As a test of the Complete MDE-DH approximation, the radial distribution function $g_{i j}(r)$ has been calculated for a system with rather large ions, $a=6.6 \AA$, and high electrolyte concentration, where the core-core correlations are important and the charge density distribution $\rho(r)$ is oscillatory. For this system $\beta_{R}=1.10$. The results for $g_{i j}$ and $\Delta g=g_{+-}-g_{--}$are presented in Fig. 7, where they are compared to MC simulations results of Zwanikken and coworkers. ${ }^{61}$ As seen in the figure, the MDE$\mathrm{DH}$ approximation gives excellent results that nearly coincide with the MC ones. Note that the resolution of the ordinate scale in frame (d) of the figure is an order of magnitude larger than in frame (a). It is noteworthy that quite intricate details of the pair distribution functions are obtained in agreement with simulations. Our results are, in fact, slightly better than those of both the HNC approximation and Zwanikken's DHEMSA approximation, ${ }^{61}$ which is quite remarkable considering the simplicity of the present Ansatz.

In order to investigate the effects of core-core correlations in this system, the pair distribution $g^{\text {hs }}(r)$ of the hard sphere fluid is plotted in Fig. 7a and it is seen that the main oscillation of $g_{i j}(r)$ with a wave length $\approx a$ is caused by these correlations. It is also seen in frames (a) and (b) of the figure that there is another 

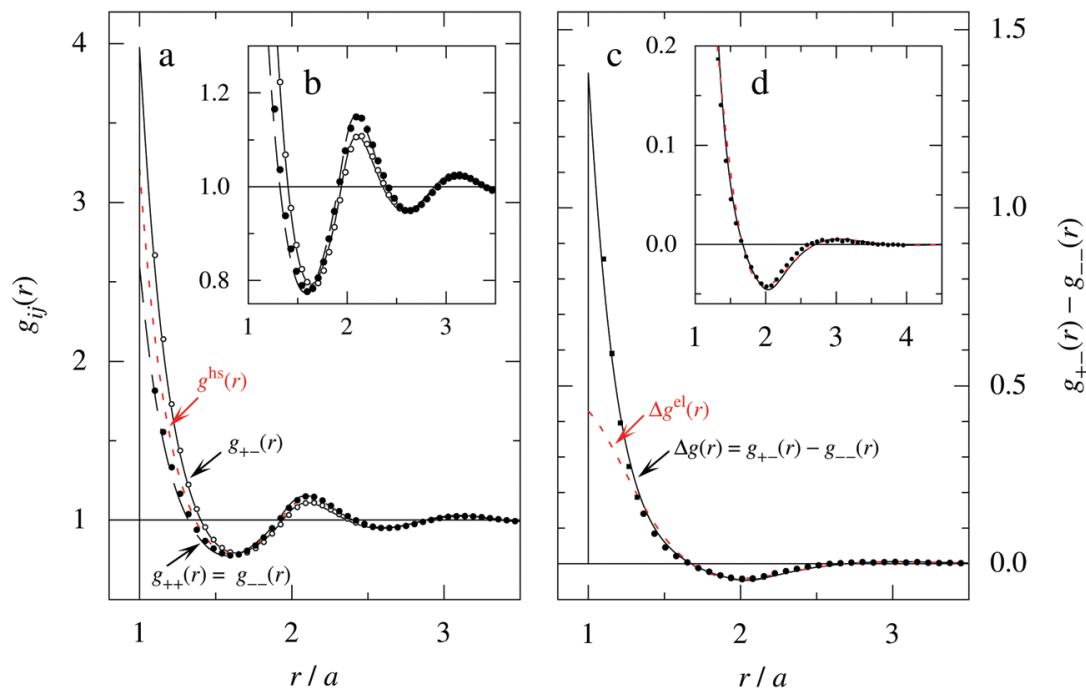

Fig. 7 (a) The radial distribution function $g_{i j}(r)$ for a $2 \mathrm{M} 1: 1$ electrolyte with ions of diameter $a=6.6 \AA$ ( $\tau=\kappa_{\mathrm{D}} a=3.11$ and $\beta_{R}=1.10$ ) calculated in the Complete MDE-DH approximation (black curves) compared with data from MC simulations (symbols) taken from ref. 61 . The red curve (short dashes) shows the pair distribution $g^{\text {hs }}(r)$ for a hard sphere fluid of the same density and sphere diameter (packing fraction $\eta=0.37$ ). The insert (b) shows a magnified view of the $g_{i j}(r)$ curves in frame (a). (c) The difference $\Delta g(r)=g_{+-}(r)-_{--}(r)$, which is proportional to the radial charge-density distribution $\rho_{-}(r)$ around a negative ion. Full curve is the MDE-DH and symbols are the MC results [the full circles are from $\Delta g(r)$ data of ref. 61 and the full squares are calculated from $g_{i j}(r)$ in frame (a) taken from the same reference]. The red curve (short dashes) shows $\Delta g^{\text {el }}(r)=2 \beta w^{\text {el }}(r)$, which is the electrostatic part of $\Delta g$ in the present approximation. The insert (d) shows a magnified view of the curves in frame (c). Note the large differences in ordinate scales of the various frames.

oscillatory component with longer wave length superimposed on the main oscillation. This component is equal to the term $\mp \beta w^{\mathrm{el}}(r)$ in eqn (56), which is oscillatory here.*** It makes the $g_{+-}$and $g_{--}$curves to repeatedly intersect with one another and has a wave length $2 \pi / \kappa_{\mathfrak{I}}=2.06 a$. In frames (c) and (d) this component gives the contribution $\Delta g^{\mathrm{el}}(r)=$ $2 \beta w^{\mathrm{el}}(r)$, which is the electrostatic part of $\Delta g$ in the current approximation. For $r \gtrsim 1.3 a$ it is seen that $\Delta g^{\mathrm{el}}(r)$ gives nearly the entire $\Delta g(r)$.

As seen in eqn (56), $g_{i j}(r)$ has also contributions from $\left[\beta w^{\mathrm{el}}(r)\right]^{2} / 2$ and from the two cross-terms involving $h^{\text {core }}$ and $w^{\mathrm{el}}$. All these contributions have shorter decay lengths than the other ones, so they mainly contribute for small $r$.

For the present system we have $\left|\mathcal{E}_{\mathrm{r}}^{\text {eff }}\right| / \varepsilon_{\mathrm{r}}=2.77$ and $\vartheta=$ -0.828 radians as obtained from eqn (109) and (110) in Appendix B. This can be compared with the values obtained from the Simple MDE-DH approximation, eqn (48) and (49), which are 1.77 and -0.477 , respectively. Eqn (58) of the latter approximation gives $g_{i j}$ functions for this system that do not agree well with the MC data in Fig. 7. This shows the importance of using the Complete MDE-DH approximation here. All other numerical results obtained so far and those in Section 4.3 below are obtained for systems with smaller ions where it is sufficient to use the simple MDE-DH approximation based on the Ansatz (43) and eqn (54), (57) and (58).

*** In the present case $\beta w^{\mathrm{el}}(r)$ is equal to the square bracket in eqn (107) in Appendix A.

\subsection{Thermodynamic quantities}

Let us now turn to the thermodynamic properties and we start with the chemical potential $\mu_{i}$ of an ion of species $i$

$$
\mu_{i}=\mu_{i}^{\text {ideal }}+\mu_{i}^{\text {ex }}=k_{\mathrm{B}} T \ln \left(\lambda_{i}{ }^{3} n_{i}\right)+\mu_{i}^{\text {ex }},
$$

where $\lambda_{i}$ is the thermal de Broglie wave length for species $i$ and $\mu_{i}^{\text {ex }}$ is the excess chemical potential. The latter has two parts $\mu_{i}^{\text {ex }}=\mu_{i}^{\text {ex,core }}+\mu_{i}^{\text {ex,el }}$, where $\mu_{i}^{\text {ex,core }}$ is the contribution from the formation of a hole in the electrolyte that is large enough to fit an ion and $\mu_{i}^{\text {ex,el }}$ is the contribution from the electrostatic interactions. We define $\mu_{i}^{\text {ex,core }}$ as the change in free energy when an uncharged sphere of diameter $a$ is inserted into the electrolyte and $\mu_{i}^{\text {ex,el }}$ as the free energy change when this sphere is charged from 0 to $q_{i}$. The average excess chemical potential $\mu_{ \pm}^{\mathrm{ex}}$ is given by $\mu_{ \pm}^{\mathrm{ex}}=\sum_{i} x_{i} \mu_{i}^{\mathrm{ex}}$ where $x_{i}=n_{i} / n_{\mathrm{tot}}$. The mean activity coefficient $\gamma_{ \pm}$is defined from $\ln \gamma_{ \pm} \equiv \mu_{ \pm}^{\mathrm{ex}} / k_{\mathrm{B}} T$ and we likewise define $\ln \gamma_{ \pm}^{\text {el }} \equiv \mu_{ \pm}^{\text {ex,el }} / k_{\mathrm{B}} T$ and $\ln \gamma_{ \pm}^{\text {core }} \equiv \mu_{ \pm}^{\text {ex,core }} / k_{\mathrm{B}} T$. The latter is equal to $\mu_{i}^{\text {ex,core }} / k_{\mathrm{B}} T$ for any $i$ because all ions have the same size. Obviously, $\ln \gamma_{ \pm}=\ln \gamma_{ \pm}^{\text {el }}+\ln \gamma_{ \pm}^{\text {core }}$.

Let us start with the core term and consider the excluded volume hole in the electrolyte where an uncharged sphere of diameter $a$ can fit. This volume, where the centers of the surrounding ions cannot enter, is equal to $4 \pi a^{3} / 3$. In the DH and MDH approximations

$$
\ln \gamma_{ \pm}^{\mathrm{core}}=\frac{4 \pi a^{3} n_{\mathrm{tot}}}{3} \quad(\mathrm{DH}, \mathrm{MDH})
$$

which can be understood from the fact that $k_{\mathrm{B}} T \ln \gamma_{ \pm}^{\text {core }}=$ $k_{\mathrm{B}} T n_{\text {tot }} \times 4 \pi a^{3} / 3$ is the reversible pressure-volume work done 
when a hole of radius $a$ and volume $4 \pi a^{3} / 3$ is formed in a lowdensity fluid and the density around the hole is equal to $n_{\text {tot }}$ throughout. As we have seen, in these approximations the total ion density around an ion is equal to the bulk value $n_{\text {tot }}$ for all distances from the ion and the same applies to an uncharged sphere or a hole.

For high ion densities it is, of course, not reasonable to evaluate $\ln \gamma_{ \pm}^{\text {core }}$ as if the total density around an uncharged sphere is unaffected by the interactions with the latter. In the MDE-DH theories for the RPM, an uncharged sphere does not interact electrostatically with the ions because $w^{\text {el }}$ is proportional to the charge of the central particle, but hard core interactions are included because of the presence of the $g^{\text {core }}$ in the pair distribution function in eqn (53). Therefore, $\ln \gamma_{ \pm}^{\text {core }}$ is equal to the excess chemical potential for the creation of a hole in a dense fluid of hard spheres of number density $n^{\text {hs }}$, which is set equal to the total ion density $n_{\text {tot }}$. Since we use the Verlet-Weis' semi-empirical expression for $g^{\text {hs }}$ and set $g^{\text {core }}=g^{\text {hs }}$ we have

$$
\ln \gamma_{ \pm}^{\text {core }}=\left.\frac{\mu_{\mathrm{CS}}^{\mathrm{ex}}}{k_{\mathrm{B}} T}\right|_{n^{\mathrm{hs}}=n_{\mathrm{tot}}}
$$

where $\mu_{\mathrm{CS}}^{\mathrm{ex}}$ is given by the very accurate Carnahan-Starling expression for $\mu^{\text {ex,core }}$ obtained from the CS equation of state ${ }^{97}$

$$
\frac{\mu_{\mathrm{CS}}^{\mathrm{ex}}}{k_{\mathrm{B}} T}=\frac{8 \eta-9 \eta^{2}+3 \eta^{3}}{(1-\eta)^{3}} \quad \text { (hard sphere fluid), }
$$

where $\eta$ is the packing fraction $\eta=\pi n^{\text {hs }} a^{3} / 6$. The value of $\ln \gamma_{ \pm}^{\text {core }}$ from eqn (60) approaches that of eqn (59) when the concentration is decreased to zero.

The electrostatic parts of the chemical potential and the activity coefficient can be calculated from the radial charge distribution functions of the electrolyte. In the DH approximation we have

$$
\ln \gamma_{ \pm}^{\mathrm{el}}=-\frac{\kappa_{\mathrm{D}}^{2}}{8 \pi n_{\mathrm{tot}}} \cdot \frac{\kappa_{\mathrm{D}}}{1+\kappa_{\mathrm{D}} a} \quad(\mathrm{DH})
$$

and in the MDH approximation ${ }^{32}$

$$
\ln \gamma_{ \pm}^{\mathrm{el}}=-\frac{\kappa_{\mathrm{D}}^{2}}{8 \pi n_{\mathrm{tot}}} \cdot \frac{\kappa}{1+\kappa a} \quad(\mathrm{MDH})
$$

with $\kappa$ from eqn (33). For a binary electrolyte where the ionic charges are $q_{+}$and $q_{-}$, the prefactor of these expressions can alternatively be written as

$$
\frac{\kappa_{\mathrm{D}}^{2}}{8 \pi n_{\mathrm{tot}}}=\frac{\beta q_{+}\left|q_{-}\right|}{8 \pi \varepsilon_{\mathrm{r}} \varepsilon_{0}}
$$

where it is clearly seen that the prefactor is a constant that is independent of the ion density.

In the Simple MDE-DH approximation based on the Ansatz (43) and eqn (58), it is shown in Appendix C that

$$
\ln \gamma_{ \pm}^{\mathrm{el}}=-\frac{\kappa_{\mathrm{D}}^{2}}{8 \pi n_{\mathrm{tot}}}\left[\frac{\varepsilon_{\mathrm{r}} \kappa}{\mathcal{E}_{\mathrm{r}}^{\mathrm{eff}}(1+\kappa a)}+\frac{\varepsilon_{\mathrm{r}} \kappa^{\prime}}{\mathcal{E}_{\mathrm{r}}^{\text {eff }}\left(1+\kappa^{\prime} a\right)}\right]
$$

and we see that there is one contribution from each of the two decay modes with decay parameters $\kappa$ and $\kappa^{\prime}$. This expression is valid also for complex-valued $\kappa$ and $\kappa^{\prime}$, but for that case one can write it in a more convenient form given by eqn (114) in Appendix C.

In any approximate theory where the charge density $\rho_{i}$ is proportional to $q_{i}$, like the $\mathrm{DH}, \mathrm{MDH}$ and MDE-DH approximations, $\mu_{ \pm}^{\text {ex,el }}$ is equal to the excess (interactional) average energy per ion, $U^{\text {ex }} / N_{\text {tot }}$ (see eqn (115) ff. in Appendix C), where $N_{\text {tot }}=\sum_{i} N_{i}=V n_{\text {tot }}$ is the total number of ions and $V$ is the volume of the system. We therefore have $\ln \gamma_{ \pm}^{\mathrm{el}}=\beta U^{\mathrm{ex}} / N_{\text {tot }}$ in these approximations, so in the the Simple MDE-DH approximation we have

$$
\frac{\beta U^{\mathrm{ex}}}{N_{\mathrm{tot}}}=-\frac{\kappa_{\mathrm{D}}^{2}}{8 \pi n_{\mathrm{tot}}}\left[\frac{\varepsilon_{\mathrm{r}} \kappa}{\mathcal{E}_{\mathrm{r}}^{\mathrm{eff}}(1+\kappa a)}+\frac{\varepsilon_{\mathrm{r}} \kappa^{\prime}}{\mathcal{E}_{\mathrm{r}}^{\prime \text { eff }}\left(1+\kappa^{\prime} a\right)}\right] .
$$

The equality between $\mu_{ \pm}^{\text {ex,el }}$ and $U^{\text {ex }} / N_{\text {tot }}$ is not true in general, but for conditions where linear electrostatic response is a good approximation we have $\ln \gamma_{ \pm}^{\mathrm{el}} \approx \beta U^{\mathrm{ex}} / N_{\text {tot }}$.

In Fig. 8a, $\ln \gamma_{ \pm}$obtained in the Simple MDE-DH approximation is compared with results from MC simulations and we see that the agreement is very good for all concentrations; there are slight differences between the results for high concentrations that are hard to see in the plot. The red portions of the curves indicate the region where the radial charge distribution is oscillatory and the Kirkwood cross-over point is shown by red arrows. The $\mathrm{DH}$ prediction is also plotted and serves as a reference in the figure. The electrostatic contribution $\ln \gamma_{ \pm}^{\text {el }}$ from these approximations is shown and these curves also give the predicted average energy plotted as $\beta U^{\mathrm{ex}} / N_{\text {tot }}$. The latter quantity as obtained from MC simulations is plotted as blue triangles in the figure and the Simple MDE-DH approximation results are very good agreement with the MC data; the slight differences seen for high concentrations are actually nearly the same as for the $\ln \gamma_{ \pm}$results in the figure.

Fig. 9a shows the low concentration region in more detail, where the results from the $\mathrm{MDH}$ approximation are also included. The latter cannot give any prediction at high ion densities (beyond the Kirkwood cross-over point) because there is only one term for the Ansatz in eqn (30) and, as seen in the figure, the $\mathrm{MDH}$ approximation works well only for concentrations up to about $0.25 \mathrm{M}\left(c_{\text {salt }}^{1 / 2} \approx 0.5 \mathrm{M}^{1 / 2}\right)$. Again we see that it is very important to have the two decay modes present in the theory in order to describe the electrolyte in an appropriate manner. The dashed-dotted curve is calculated by using $\ln \gamma_{ \pm}^{\text {core }}$ from eqn (59) rather than from eqn (60). By comparing the full and the dashed-dotted curves we can see that for concentrations below about $0.25 \mathrm{M}\left(c_{\text {salt }}^{1 / 2} \approx 0.5 \mathrm{M}^{1 / 2}\right)$, it it does not matter which expression one uses, but for higher concentrations one must use the CS expression in order to obtain the correct values for $\ln \gamma_{ \pm}$.

The osmotic coefficient $\phi$ is defined from $\phi=P / P^{\text {ideal }}$ where $P$ is the (osmotic) pressure of the fluid and $P^{\text {ideal }}=k_{\mathrm{B}} T n_{\text {tot }}$ is the ideal pressure. The excess pressure is $P^{\mathrm{ex}}=P-P^{\text {ideal }}$ and the excess osmotic coefficient is given by $\phi^{\text {ex }}=P^{\text {ex }} / P^{\text {ideal }}=\phi-1$ (see eqn (116) ff. in Appendix C). In the $\mathrm{DH}$ approximation 

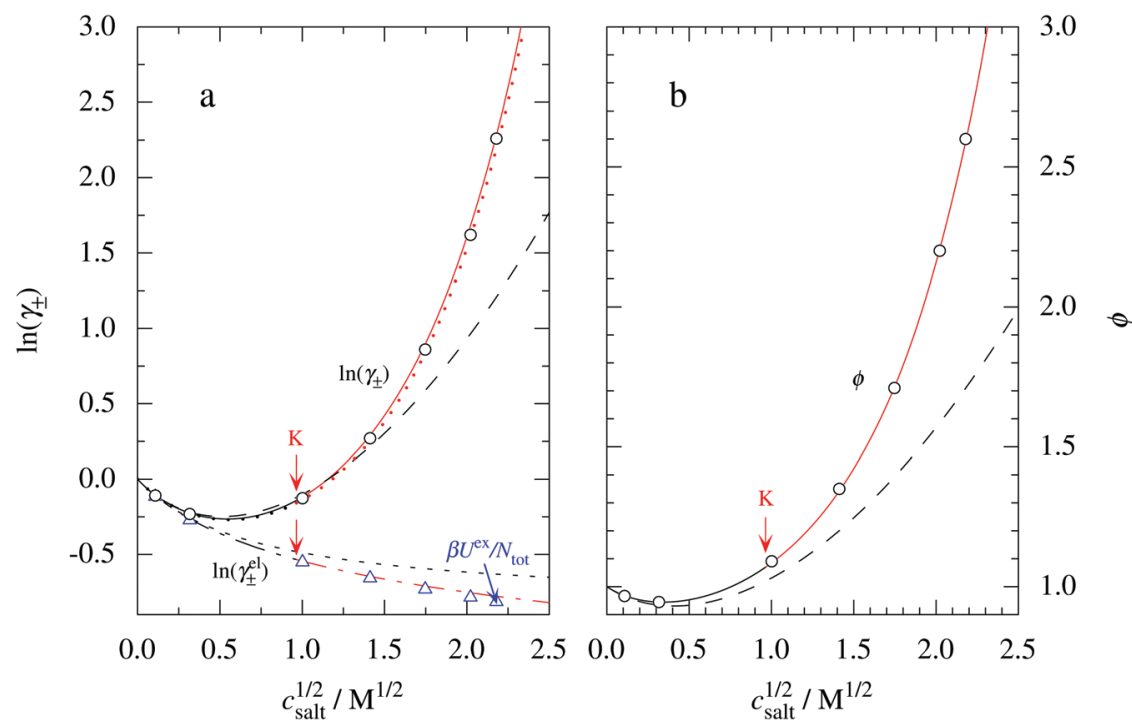

Fig. 8 (a) The logarithm of the mean activity coefficient $\gamma_{ \pm}$and its electrostatic part $\gamma_{ \pm}^{\text {el }}$ plotted as functions of the square root of the salt concentration $c_{\text {salt }}$ for $1: 1$ aqueous electrolyte solutions $\left(a=4.25 \AA, T=298 \mathrm{~K}, \beta_{R}=1.68\right)$. The full and dotted curves show $\ln \gamma_{ \pm}$from the Simple MDE-DH approximation; the full curve is obtained directly and the dotted one is obtained via an integration of the osmotic coefficient. The symbols show the results from MC simulations taken from ref. 77. The alternatingly dashed-minidashed curve shows $\ln \gamma_{ \pm}^{\mathrm{el}}=\beta U^{\mathrm{ex}} / N_{\text {tot }}$ from the Simple $M D E-D H$ approximation (in this approximation $\mu_{ \pm}^{\text {ex,el }}=k_{\mathrm{B}} T \ln \gamma_{ \pm}^{\text {el }}$ is equal to $U^{\text {ex }} / N_{\text {tot }}$ ). The blue triangles show $\beta U^{\text {ex }} / N_{\text {tot }}$ from the same MC simulations (In $\gamma_{ \pm}^{\text {el }}$ is in general not equal to $\beta U^{\mathrm{ex}} / N_{\text {tot }}$ in simulations). The Kirkwood cross-over point (K) between monotonic and oscillatory decay is indicated by red arrows; for the red portions of the curves the radial charge distribution is oscillatory. For reference, we also show the Debye-Hückel (DH) predictions for $\ln \gamma_{ \pm}$and $\ln \gamma_{ \pm}^{\text {el }}=\beta U^{\mathrm{ex}} / N_{\text {tot }}$ as dashed curves. (b) The osmotic coefficient $\phi$ plotted in the same manner. The full curve shows Simple MDE-DH, symbols MC and dashes $\mathrm{DH}$ results.

we have

$$
\phi^{\mathrm{ex}}=\phi^{\mathrm{cont}}+\phi^{\mathrm{el}}=\frac{2 \pi a^{3} n_{\mathrm{tot}}}{3}-\frac{\kappa_{\mathrm{D}}^{2}}{24 \pi n_{\mathrm{tot}}} \cdot \frac{\kappa_{\mathrm{D}}}{1+\kappa_{\mathrm{D}} a}
$$

and in the MDH approximation ${ }^{32}$

$$
\phi^{\mathrm{ex}}=\phi^{\mathrm{cont}}+\phi^{\mathrm{el}}=\frac{2 \pi a^{3} n_{\mathrm{tot}}}{3}-\frac{\kappa_{\mathrm{D}}^{2}}{24 \pi n_{\mathrm{tot}}} \cdot \frac{\kappa}{1+\kappa a}
$$

$(\mathrm{MDH})$,

where the first term in each rhs is the contribution $\phi^{\text {cont }}=P^{\text {cont }} /$ $P^{\text {ideal }}$ from the contact pressure $P^{\text {cont }}$ at the hard core surfaces of the ions, the second term is $\phi^{\mathrm{el}}=P^{\mathrm{el}} / P^{\mathrm{ideal}}$ and $P^{\mathrm{el}}$ is the pressure from the Coulombic interactions in the electrolyte.

As shown in Appendix C, in the Simple MDE-DH approximation we have

$$
\phi^{\mathrm{cont}}=\phi^{\mathrm{core}}+\frac{\pi a^{3} n_{\mathrm{tot}}}{3}\left[\beta w^{\mathrm{el}}(a)\right]^{2}
$$

where $\phi^{\text {core }}=2 \pi a^{3} n_{\text {totg }} g^{\text {core }}(a) / 3$, and

$$
\phi^{\mathrm{el}}=-\frac{\kappa_{\mathrm{D}}^{2}}{24 \pi n_{\mathrm{tot}}}\left[\frac{\varepsilon_{\mathrm{r}} \kappa}{\mathcal{E}_{\mathrm{r}}^{\mathrm{eff}}(1+\kappa a)}+\frac{\varepsilon_{\mathrm{r}} \kappa^{\prime}}{\mathcal{E}_{\mathrm{r}}^{\prime \text { eff }}\left(1+\kappa^{\prime} a\right)}\right] .
$$

Since $g^{\text {core }}(r)=g^{\text {hs }}(r)$ we have $\phi^{\text {core }}=\phi^{\text {ex,hs }}=\phi^{\text {hs }}-1$, that is, the excess osmotic coefficient for a pure hard sphere fluid of density $n^{\text {hs }}=n_{\text {tot }}$. The use of the Verlet-Weis' expression for $g^{\text {hs }}$ implies that this part of the osmotic coefficient is given by the Carnahan-Starling equation of state, which says that

$$
\phi_{\mathrm{CS}}^{\mathrm{hs}}=\frac{1+\eta+\eta^{2}-\eta^{3}}{(1-\eta)^{3}} \quad \text { (hard sphere fluid), }
$$

so we have

$$
\phi^{\text {core }}=\left.\phi_{\mathrm{CS}}^{\mathrm{hs}}\right|_{n^{\mathrm{hs}=n \text { tot }}}-1
$$

in eqn (68). When the ion density goes to zero, this $\phi^{\text {core }}$ goes to $\phi^{\text {cont }}$ of the DH and MDH approximations in eqn (66) and (67). The latter only include the core contribution to $\phi^{\text {cont }}$.

The prediction for $\phi$ obtained in the simple MDE-DH approximation is shown as the full curve in Fig. $8 \mathrm{~b}$ and the low concentration region is shown in Fig. 9b. It is seen that this prediction is in very good agreement with the MC data for all concentrations. Again, the curve nearly coincide with the MC data.

The activity coefficient can alternatively be obtained from the osmotic coefficient via the relationship

$$
\ln \gamma_{ \pm} \equiv \frac{\mu_{ \pm}^{\mathrm{ex}}}{k_{\mathrm{B}} T}=\phi^{\mathrm{ex}}+2 \int_{0}^{\sqrt{n_{\mathrm{tot}}}} d\left(\sqrt{n_{\mathrm{tot}}{ }^{\prime}}\right) \frac{\phi^{\mathrm{ex}}\left(n_{\mathrm{tot}}{ }^{\prime}\right)}{\sqrt{n_{\mathrm{tot}}{ }^{\prime}}}
$$

which can be derived from the Gibbs-Duhem equation (the selected integration variable $\sqrt{n_{\text {tot }}{ }^{\prime}}$ is suitable since $\phi^{\text {ex }}\left(n_{\text {tot }}{ }^{\prime}\right)$ is proportional to $\sqrt{n_{\mathrm{tot}}{ }^{\prime}}$ for small densities). In the integrand, $\phi^{\mathrm{ex}}\left(n_{\text {tot }}{ }^{\prime}\right)$ is evaluated for an electrolyte with total density $n_{\text {tot }}{ }^{\prime}$ that varies from 0 to the final value while keeping the mole fraction $x_{i}$ constant, whereby one sets the ion density equal to $n_{i}^{\prime}=x_{i} n_{\text {tot }}{ }^{\prime}$ when calculating $\phi^{\text {ex }}$. 

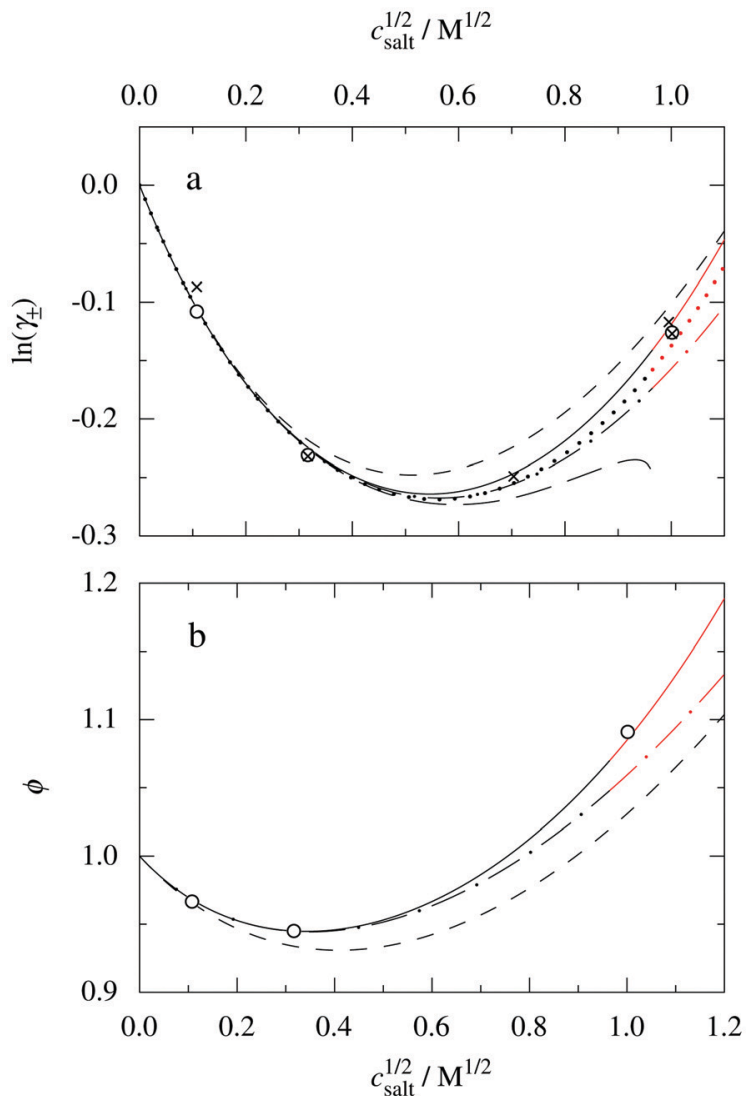

Fig. 9 The quantities (a) $\ln \gamma_{ \pm}$and (b) $\phi$ for the same system as in Fig. 8 , but displayed in an expanded view for low concentrations. The full, dotted and short-dashed curves and the open circles are the same as in Fig. 8. In frame (a), the crosses show MC simulation data from ref. 72 and the longdashed curve shows the result of the MDH approximation. The dasheddotted curves in both frames are based on the Ansatz (43), like the full and dotted curves, but they are calculated with the same hard core contribution for $\ln \gamma_{ \pm}$and $\phi$ as the $\mathrm{DH}$ and $\mathrm{MDH}$ approximations. The red portions of the curves indicate the region where the radial charge distribution is oscillatory

In approximate theories, there is no guarantee that $\ln \gamma_{ \pm}$ calculated from $\phi^{\text {ex }}$ via eqn (71) is the same as $\ln \gamma_{ \pm}$obtained earlier. In order to have consistency, one would need to obtain the same value of each quantity irrespectively of how it is calculated, but this is guaranteed only in exact theory. A well-known fact is that the $\mathrm{DH}$ approximation shows very poor consistency for $\ln \gamma_{ \pm}$and the same is true for the $\mathrm{MDH}$ approximation. $^{32}$ The use of eqn (71) is discussed in Appendix $\mathrm{C}$, where we see that it is a common feature of all linear approximations (where $\rho_{i}$ is proportional $q_{i}$ ) that the contribution $\phi^{\mathrm{el}}$ in $\phi^{\mathrm{ex}}$ integrated according to eqn (71) does not give ln $\gamma_{ \pm}^{\mathrm{el}}$. In the $\mathrm{DH}$ and $\mathrm{MDH}$ approximations, this is the cause of the inconsistency for $\ln \gamma_{ \pm}$.

In the Simple MDE-DH approximation, the contribution $\phi^{\mathrm{el}}$ does not give $\ln \gamma_{ \pm}^{\text {el }}$ via this integration for the same reason. However, there is also an electrostatic contribution from the square term in $\phi^{\text {cont }}$ given by eqn (68) that originates from the square term in eqn (58). This contribution makes the situation different. In Fig. 8a the dotted curve shows $\ln \gamma_{ \pm}$obtained via the integration (71) from the osmotic coefficient in Fig. 8b. In Fig. 9a the low concentration region is shown in more detail. The dotted curve agrees well with the full curve, so we see that this approximation shows a good thermodynamic consistency in this respect. Considering that the theory is based on the very simple Ansatz (43) and eqn (58), this is an accomplishment. In fact, it is not uncommon for more advanced theories to lack such a consistency.

For the system in Fig. 7 with large ions, the Complete MDE-DH approximation given by eqn (54) and (55) is, as we have seen, needed for the distribution functions because the cross-terms in eqn (56) give non-negligible contributions. However, when the thermodynamic quantities of this system are calculated in this approximation, $\ln \gamma_{ \pm}$deviates only by $0.4 \%$ and $\phi$ by $0.9 \%$ from the predictions by the Simple MDEDH approximation, so the latter can still be used as a good approximation for these quantities. This is a further example of the fact that thermodynamical quantities sometimes are not overly sensitive to the details of the structural entities like the distribution functions. The corresponding deviations for the contributions $\ln \gamma_{ \pm}^{\mathrm{el}}$ and $\phi^{\mathrm{el}}$ are $5.5 \%$ for both. $\dagger \dagger \dagger$ If the latter quantities are needed with a higher accuracy than this, one has, of course, to use the Complete MDE-DH approximation. The formulas for such calculations are presented in Appendix C, eqn (125)-(127).

\section{Influence of terms with higher powers of $w_{i j}$}

As we have seen, the approximations in this paper are based on the basic Ansatz (52)

$$
g_{i j}(r)=\mathrm{e}^{-\beta w_{i j}(r)}=\mathrm{e}^{-\beta\left[w_{i j}^{\mathrm{el}}(r)+w_{i j}^{\text {core }}(r)\right]}=\mathrm{e}^{-\beta w_{i j}^{\mathrm{el}}(r)} g_{i j}^{\text {core }}(r),
$$

where $g_{i j}^{\text {core }}=\mathrm{e}^{-\beta w_{i j}^{\text {core }}}$ is set equal to $g^{\mathrm{hs}}$ and $w_{i j}^{\mathrm{el}}$ is given by the Ansatz (28) with two Yukawa function terms. The electrostatic part of $g_{i j}$ can be expressed as a Taylor expansion

$$
\mathrm{e}^{-\beta w_{i j}^{\mathrm{el}}}=1-\beta w_{i j}^{\mathrm{el}}+\frac{\left(\beta w_{i j}^{\mathrm{el}}\right)^{2}}{2 !}-\frac{\left(\beta w_{i j}^{\mathrm{el}}\right)^{3}}{3 !}+\ldots
$$

For the RPM, we have $w_{+ \pm}^{\mathrm{el}}(r)= \pm w^{\mathrm{el}}(r)$ with $w^{\mathrm{el}}$ given by eqn (54). In the MDE-DH approximations we only keep terms up to and including the quadratic one in the expansion (72). This leads to eqn (55), which contains a square term that does not contribute to $\rho(r)$ so the latter is linear in $w_{i j}^{\text {el }}$. In fact, for all numerical results presented so far for the monovalent ions, the inclusion of all nonlinear terms changes the results only marginally, so the MDE-DH approximation used is fully adequate.

There remains, however, one issue to be considered. For dilute electrolytes where the screening is low, $w_{i j}^{\mathrm{el}}(r)$ has a long range and the terms with higher powers than two have, in fact, a qualitative effect on the screening behavior. In the limit of infinite dilution we have the exact limiting law in eqn (5) for the

$\dagger \dagger \dagger$ In absolute values, $\ln \gamma_{ \pm}^{\text {el }}$ give $8 \%$ of $\ln \gamma_{ \pm}$and $\phi^{\mathrm{el}} 3 \%$ of $\phi$ in this system. 
screening parameter $\kappa$, which takes the following form for symmetric binary electrolytes $\$+\ddagger$

$$
\frac{\kappa^{2}}{\kappa_{\mathrm{D}}^{2}} \sim 1+\frac{z^{4} \Lambda^{2} \ln (\Lambda)}{6} \text { when } \Lambda \rightarrow 0,
$$

where $z=q / q_{\mathrm{e}}$. This asymptotic result originates, in fact, from the term in eqn (72) with $w_{i j}^{\text {el }}$ to the third power. $\S \S$ Later we will derive an approximate extension, eqn (81), of this limiting law.

As we have seen, since the logarithm in eqn (73) is negative for small $\Lambda$, the second term in the rhs is negative there, so $\kappa<\kappa_{\mathrm{D}}$ for dilute symmetric electrolytes and there is accordingly under-screening. However, the approximate formula (33) for $\kappa$ in the MDE-DH approximation implies over-screening, $\kappa>\kappa_{\mathrm{D}}$, and when $\kappa_{\mathrm{D}} \rightarrow 0$ it gives $\left(\kappa / \kappa_{\mathrm{D}}\right)^{2} \sim 1+(\kappa a)^{2} / 2 \sim 1+$ $\left(\kappa_{\mathrm{D}} a\right)^{2} / 2$. Therefore, this $\kappa$ is qualitatively incorrect for very dilute solutions in this approximation. Other linear approximations for the electrostatic response in electrolytes, like MSA, LMPB and GDH, share the same features; they all predict that $\kappa \geq \kappa_{\mathrm{D}}$ and a limiting form proportional to $\left(\kappa_{\mathrm{D}} a\right)^{2}$.

As mentioned in the Introduction, it has been experimentally observed that $\kappa<\kappa_{\mathrm{D}}$ in dilute solutions of symmetric electrolytes at high electrostatic coupling, so this phenomenon is relevant to investigate. Since eqn (73) has general validity as an exact limiting law, it is applicable also for low electrostatic coupling so it is theoretically interesting to start with this latter case.

In order to analyze these matters, let us investigate the predictions of the full nonlinear expression for $\rho(r)=q n\left[g_{++}(r)\right.$ $\left.-g_{+-}(r)\right]$ with $g_{i j}(r)$ given by eqn (53) and (54), that is,

$$
\rho(r)=-q n_{\mathrm{tot}} \sinh \left[\frac{\beta\left(q^{\mathrm{eff}}\right)^{2}}{4 \pi \mathcal{E}_{\mathrm{r}}^{\mathrm{eff}} \varepsilon_{0}} \cdot \frac{\mathrm{e}^{-\kappa r}}{r}+\frac{\beta\left(q^{\prime}{ }^{\mathrm{eff}}\right)^{2}}{4 \pi \mathcal{E}_{\mathrm{r}}^{\prime} \text { eff } \varepsilon_{0}} \cdot \frac{\mathrm{e}^{-\kappa^{\prime} r}}{r}\right] g_{i j}^{\text {core }}(r)
$$

for $r \geq a$ and zero otherwise. At high dilution, the contribution from the second Yukawa term with decay parameter $\kappa^{\prime}$ can be neglected because $\mathcal{E}_{\mathrm{r}}^{\prime \text { eff }}$ is very large there and $g_{i j}^{\text {core }}(r) \approx 1$, so

\#+ Note that for any constant $C>0$ we have $\Lambda^{2} \ln (C \Lambda)=\Lambda^{2} \ln (\Lambda)+\Lambda^{2} \ln (C) \sim$ $\Lambda^{2} \ln (\Lambda)$ when $\Lambda \rightarrow 0$. This means that any factor $C$ can be included in the logarithm and the limiting law is still valid. For eqn (73) to be useful apart from at high dilution, one must therefore at least add the next order asymptotic term, which proportional to $\Lambda^{2}$, that is, proportional to the ion concentration. That term, which depends on the ionic characteristics like the ionic size, specifies an appropriate $C$. No exact simple expression for that term is, however, presently known, but we will later derive an approximate expression for it, eqn (81). We may note that $z^{4} \Lambda^{2} \ln (\Lambda)=\left(\beta_{R} \tau\right)^{2} \ln \left(\beta_{R} \tau / z^{2}\right)$, which means that for two systems with different ionic valencies but with the same value of $\beta_{R}$, eqn (73) does not give the same asymptote as function of $\tau$ except in the limit of infinite dilution. This peculiarity occurs despite that the statistical mechanics in the RPM is determined solely by $\beta_{R}$ and $\tau$ and it can easily be eliminated by selecting $C=z^{2}$. Eqn (73) is, however, derived for use in the infinite dilution limit only, where the value of $C$ does not matter. A better selection of $C$ is done in eqn (81), which is valid for a larger range of concentrations; see also footnote $\mathbf{9} \mathbf{9 \top}$.

$\S \S \S$ The term $-\left(\beta w_{\mathrm{ij}}^{\text {el }}\right)^{3} / 3$ ! corresponds to the low concentration limit of the term $\left(h_{i j}^{\#}\right)^{3} / 3$ ! in eqn (103) of ref. 101 in the derivation of the exact limiting law for $\kappa^{2} / \kappa_{\mathrm{D}}^{2}$. $\rho(r)$ is given to a very good approximation by

$$
\rho(r)=-q n_{\mathrm{tot}} \sinh \left[\frac{\beta\left(q^{\mathrm{eff}}\right)^{2}}{4 \pi \mathcal{E}_{\mathrm{r}}^{\mathrm{eff}} \varepsilon_{0}} \cdot \frac{\mathrm{e}^{-\kappa r}}{r}\right] \quad \text { for } r \geq a .
$$

Approximations that are similar to eqn (75) have been investigated earlier by Attard $^{84}$ and Kjellander. ${ }^{32}$ Here the task is to use this equation to evaluate the behavior of $\kappa$ and $\mathcal{E}_{\mathrm{r}}^{\text {eff }}$ in eqn (74) for low electrolyte concentrations.

Using the exact eqn (51), we can write the coefficient in the prefactor of the argument of sinh in eqn (75) as

$$
\frac{\beta\left(q^{\mathrm{eff}}\right)^{2}}{4 \pi \mathcal{E}_{\mathrm{r}}^{\text {eff }} \varepsilon_{0}}=\frac{\beta q^{2}}{4 \pi \varepsilon_{0} \varepsilon_{\mathrm{r}}}\left[\frac{\kappa^{2}}{\kappa_{\mathrm{D}}^{2}}\right]^{2} \frac{\varepsilon_{\mathrm{r}}}{\mathcal{E}_{\mathrm{r}}^{\mathrm{eff}}}=\beta_{R} a\left[\frac{\kappa}{\kappa_{\mathrm{D}}}\right]^{4} \frac{\varepsilon_{\mathrm{r}}}{\mathcal{E}_{\mathrm{r}}^{\mathrm{eff}}} .
$$

The local electroneutrality condition and the Stillinger-Lovett second moment condition can be expressed in the RPM as

$$
\int d \mathbf{r} \rho(r)=-q
$$

and

$$
\beta q n_{\mathrm{tot}} \int d \mathbf{r} r^{2} \rho(r)=-6 \varepsilon_{\mathrm{r}} \varepsilon_{0}
$$

respectively. By inserting eqn (75) into these equations and using $n_{\text {tot }} a^{3}=\tau^{2} /\left(4 \pi \beta_{R}\right)$, we obtain the following set of equations for the two unknowns $\kappa / \kappa_{\mathrm{D}}$ and $\mathcal{E}_{\mathrm{r}}^{\mathrm{eff}} / \varepsilon_{\mathrm{r}}$ for given dimensionless system parameters $\tau$ and $\beta_{R}$

$$
\frac{\tau^{2}}{\beta_{R}} \int_{1}^{\infty} \mathrm{d} R R^{2} \sinh \left(\beta_{R}\left[\frac{\kappa}{\kappa_{\mathrm{D}}}\right]^{4} \frac{\varepsilon_{r}}{\mathcal{E}_{\mathrm{r}}^{\mathrm{eff}}} \cdot \frac{\mathrm{e}^{-\left[\frac{\kappa}{\kappa_{\mathrm{D}}}\right] \tau R}}{R}\right)=1
$$

and

$$
\frac{\tau^{4}}{6 \beta_{R}} \int_{1}^{\infty} \mathrm{d} R R^{4} \sinh \left(\beta_{R}\left[\frac{\kappa}{\kappa_{\mathrm{D}}}\right]^{4} \frac{\varepsilon_{\mathrm{r}}}{\mathcal{E}_{\mathrm{r}}^{\mathrm{eff}}} \cdot \frac{\mathrm{e}^{-\left[\frac{\kappa}{\kappa_{\mathrm{D}}}\right] \tau R}}{R}\right)=1,
$$

where we have made the variable substitution $R=r / a$. This set of equations can be solved numerically for $\kappa / \kappa_{\mathrm{D}}$ and $\mathcal{E}_{\mathrm{r}}^{\text {eff }} / \varepsilon_{\mathrm{r}}$. It constitutes an approximate nonlinear theory that is valid for dilute electrolytes. We will denote this approximation as the Extended DHX (EDHX) approximation. It is the limiting form for infinite dilution of the nonlinear (DHX) version of the MDE$\mathrm{DH}$ approximation, where $\rho(r)$ is given by eqn (74) and $g_{+ \pm}(r)=$ $\mathrm{e}^{\mp \beta w \mathrm{el}(r)} g^{\text {core }}(r)$ with $w^{\text {el }}$ from eqn (54) [cf. the discussion in Section 2 in connection to eqn (28) and (29)].

Before proceeding we note that the linearized version of this approximation is equal to the self-consistent screening length, SCSL, approximation by Attard, which was discussed in Section 3. This follows because linearization of eqn (75) gives [cf. eqn (35)]

$$
\rho(r)=-q n_{\mathrm{tot}} \frac{\beta\left(q^{\mathrm{eff}}\right)^{2}}{4 \pi \mathcal{E}_{\mathrm{r}}^{\mathrm{eff}} \varepsilon_{0}} \cdot \frac{\mathrm{e}^{-\kappa r}}{r}=-\frac{\kappa^{2} q^{\mathrm{eff}} \varepsilon_{\mathrm{r}}}{4 \pi \mathcal{E}_{\mathrm{r}}^{\mathrm{eff}}} \cdot \frac{\mathrm{e}^{-\kappa r}}{r} \quad \text { for } r \geq a
$$


and the application of the local electroneutrality and StillingerLovett conditions yields $\left(\kappa / \kappa_{\mathrm{D}}\right)^{2}$ given by eqn (38), which is the SCSL approximation. Furthermore, this linearized expression for $\rho(r)$ yields $\mathcal{E}_{\mathrm{r}}^{\text {eff }} / \varepsilon_{\mathrm{r}}$ given by eqn (36). Since the EDHX and SCSL approximations contain only one decay mode, they can be useful only for dilute electrolytes.

The numerical solution of eqn (76) and (77) of the EDHX approximation gives the following results. Fig. 10 shows the ratios $\kappa^{2} / \kappa_{\mathrm{D}}{ }^{2}$ and $\mathcal{E}_{\mathrm{r}}^{\mathrm{eff}} / \varepsilon_{\mathrm{r}}$ as functions of $\tau=\kappa_{\mathrm{D}} a$ for a monovalent electrolyte with $a=4.6 \AA$ in aqueous solution at room temperature, which corresponds to $\beta_{R}=1.55$ (recall that $\kappa_{\mathrm{D}} a$ is proportional to the square root of concentration). The predictions of the EDHX approximation are compared in the figure with results from MC simulations, the HNC, MDE-DH and SCSL approximations. In the insert to Fig. 10a, which shows a magnified view of the dilute region, we see that the EDHX approximation indeed gives $\kappa<\kappa_{\mathrm{D}}$ for the dilute electrolyte in agreement with the exact asymptotic formula and the HNC approximation. However, the slight dip of $\kappa^{2} / \kappa_{\mathrm{D}}{ }^{2}$ below 1 occurs for small ion densities where the deviation of $\kappa$ from $\kappa_{\mathrm{D}}$ is negligible in practice. Despite that the curves for MDE-DH and SCSL approximations in the insert show the qualitatively incorrect behavior with $\kappa>\kappa_{\mathrm{D}}$, it only on this greatly magnified scale that this can be seen. Likewise, in the insert to Fig. $10 \mathrm{~b}$ we can see that $\mathcal{E}_{\mathrm{r}}^{\text {eff }}>\varepsilon_{\mathrm{r}}$ for dilute solutions as shown by the HNC and EDHX approximations, while the MDE-DH and SCSL approximations give $\mathcal{E}_{\mathrm{r}}^{\text {eff }}<\varepsilon_{\mathrm{r}}$ there, which is incorrect. Also in this case, the incorrect behavior occurs for low densities where the deviation of $\mathcal{E}_{\mathrm{r}}^{\text {eff }}$ from $\varepsilon_{\mathrm{r}}$ is negligible for most practical purposes.

In the main frames of Fig. 10 we see, as before, that the MDE-DH approximation gives a very good over-all account of the properties of the monovalent electrolyte. Furthermore, we can see that the EDHX and SCSL approximations do not work for higher concentrations, because, as pointed out earlier, they only contain one decay mode. In the figure one can clearly see that it is very important to include two decay modes with different decay parameters $\kappa$ and $\kappa^{\prime}$, as in the MDE-DH approximation, in order to get agreement with the accurate calculations outside the dilute region.

The fact that $\kappa<\kappa_{\mathrm{D}}$ for low concentrations, as shown by the asymptotic formula eqn (73), is a general feature for symmetric electrolytes. Due to the factor $z^{4}$ in this equation, the underscreening will become much more pronounced when the valency of the ions is increased. This has been verified for divalent $(2: 2)$ electrolytes in ref. 18 and 22 by HNC and MC calculations; these HNC and MC results are shown in Fig. 11 together with the corresponding result from the EDHX approximation. As seen in Fig. 11a, we have $\kappa<\kappa_{\mathrm{D}}$ for the divalent case in a wide concentration interval and the negative deviation of $\kappa^{2} / \kappa_{\mathrm{D}}^{2}$ from 1 reaches about 30\% (shown by the MC data) before it rises again. This highly significant difference from the monovalent case is solely due to the difference in ionic charge by a factor of two; everything else is the same. In this case the HNC approximation (dashed curve) does not provide accurate values of $\kappa$, except for low concentrations. As pointed out earlier, the EDHX approximation is applicable only for sufficiently dilute electrolytes, but for higher concentrations it nevertheless has the correct qualitative behavior in a part of the concentration interval shown.

The results for the effective dielectric permittivity $\mathcal{E}_{\mathrm{r}}^{\text {eff }}$ for the divalent electrolytes is shown in Fig. 11b. Again, the HNC approximation provides accurate results only in the dilute region. In contrast to the monovalent case, we see that $\mathcal{E}_{\mathrm{r}}^{\text {eff }}>\varepsilon_{\mathrm{r}}$ in the
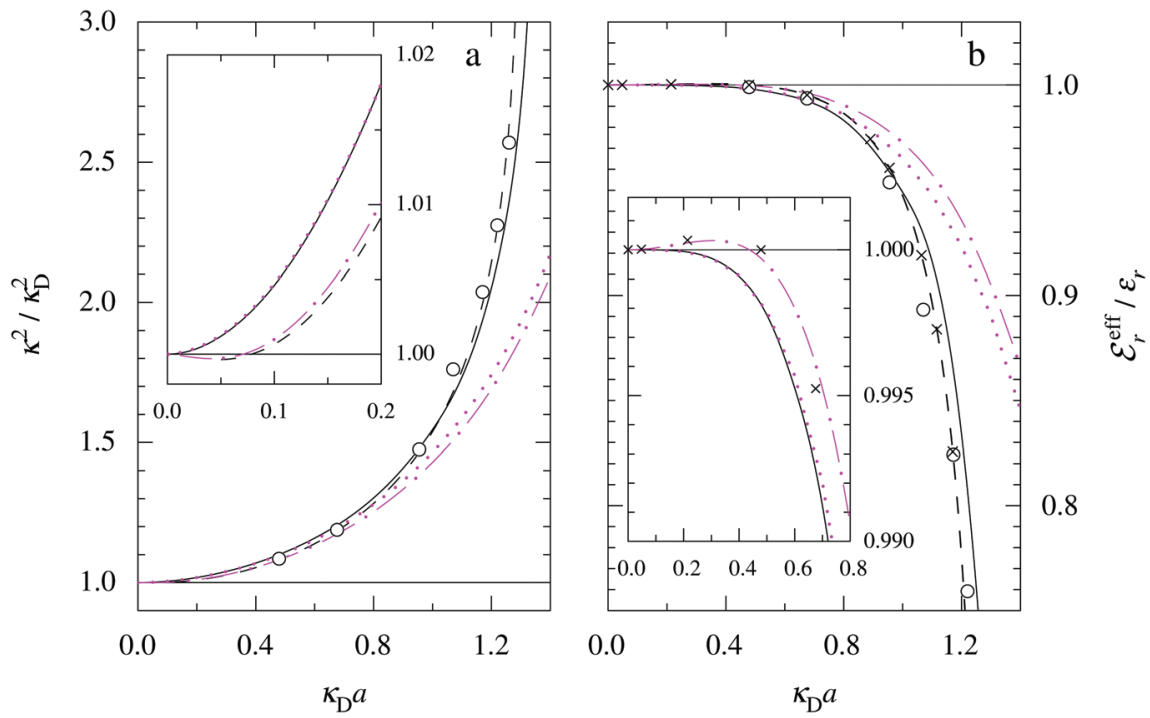

Fig. 10 The ratios (a) $\kappa^{2} / \kappa_{\mathrm{D}}^{2}$ and (b) $\mathcal{E}_{\mathrm{r}}^{\text {eff }} / \varepsilon_{\mathrm{r}}$ plotted as functions of $\kappa_{\mathrm{D}}$ a for monovalent electrolytes in aqueous solution at room temperature $\left(\beta_{R}=1.55\right)$. The respective insert shows the same plot in an expanded view for low concentrations (low $\kappa_{\mathrm{D}} a$ ). The dot-dashed curves are the result from the EDHX approximation, eqn (76) and (77), and the dotted curves are from the SCSL approximation; both are drawn in magenta color. The full curves are the Simple MDE-DH results, the dashed curves and crosses show the results from HNC calculations (the crosses occur only in b), and open circles are from MC simulations. The HNC and MC data are taken from ref. 18 and 22. 

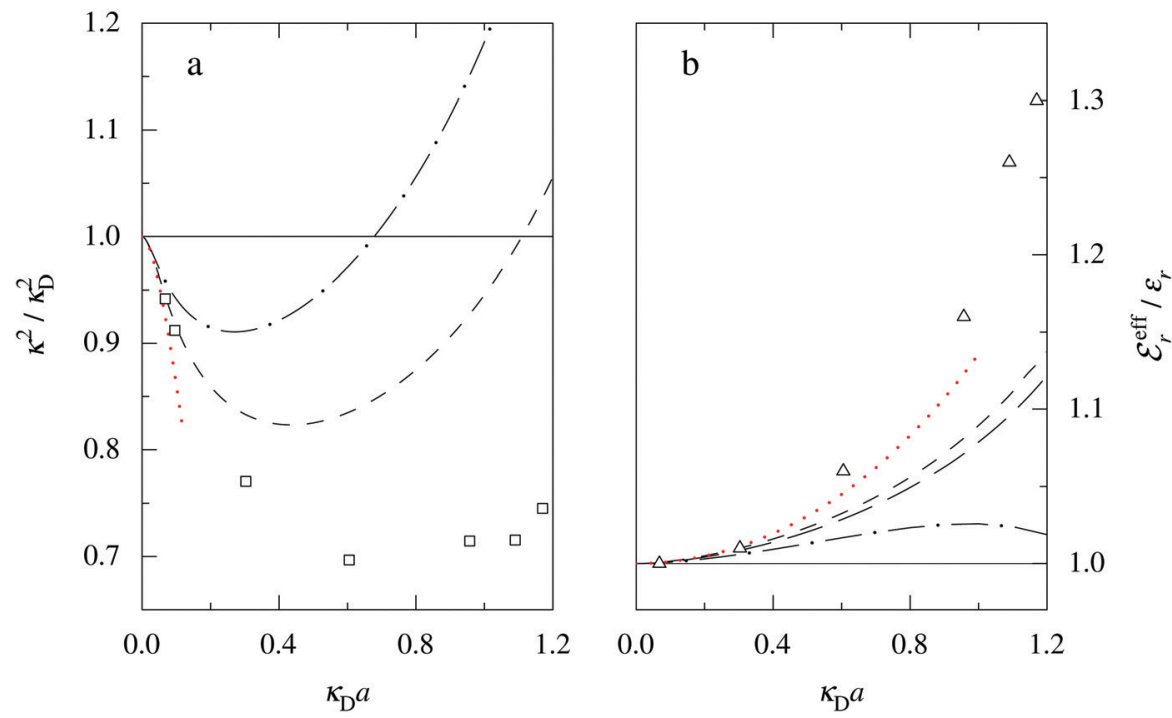

Fig. 11 Frames (a) and (b) show the same kind of plot as Fig. 10a and b, but for divalent ( $2: 2$ ) electrolytes in aqueous solution at room temperature. The symbols show the results from MC simulations, the dashed curves from HNC calculations, the dot-dashed curves from the EDHX approximation that is valid only for low concentrations, and the red dotted curves show the asymptotes given by eqn (81) and (82), respectively. The ions have diameter $a=4.6 \AA$ and $\beta_{R}=6.22$ except for the HNC results in frame (b), where $a=4.2 \AA$ (short dashes) and $5.0 \AA$ (long dashes); the corresponding HNC curve of $a=4.6 \AA$ would lie between these curves. The HNC and MC data are taken from ref. 18 and 22.

entire interval shown. The under-screening is accordingly accompanied by an augmentation of the effective dielectric permittivity of the electrolyte caused by the strongly nonlinear ion-ion correlations. For $1: 1$ electrolytes we have $\mathcal{E}_{\mathrm{r}}^{\text {eff }}>\varepsilon_{\mathrm{r}}$ only in a small part of the very dilute region in the insert to Fig. 10b. Furthermore, we saw in Fig. 3 for the latter case, that $\mathcal{E}_{\mathrm{r}}^{\text {eff }} / \varepsilon_{\mathrm{r}}$ quickly approaches $\varepsilon_{\mathrm{r}}$ from below when $\kappa_{\mathrm{D}} a$ is decreased from the Kirkwood cross-over point and then stays nearly constant $\mathcal{E}_{\mathrm{r}}^{\text {eff }} \approx \varepsilon_{\mathrm{r}}$ when $\kappa_{\mathrm{D}} a \rightarrow 0$. In contrast, for the $2: 2$ case in Fig. 11, $\mathcal{E}_{\mathrm{r}}^{\text {eff }}$ approaches $\varepsilon_{\mathrm{r}}$ from above in a slow manner when $\kappa_{\mathrm{D}} a \rightarrow 0$. Incidentally, we may note that for high values of $\kappa_{\mathrm{D}} a$ (outside the figure) there is a Kirkwood cross-over point at $\kappa_{\mathrm{D}} a \approx 1.7$ for the $2: 2$ case with $a=4.6 \AA$ and $\mathcal{E}_{\mathrm{r}}^{\text {eff }}$ decreases quickly to zero when that point is approached, see ref. 18 and 22 .

The nonlinear electrostatic effects in ion-ion correlations are important for any system where the electrostatic coupling is appreciably stronger than the monovalent case we have investigated, that is, for lower temperatures, lower $\varepsilon_{\mathrm{r}}$ or higher valencies. This means that terms in higher powers of $w_{i j}^{\mathrm{el}}$ than the square one in eqn (72) cannot be neglected and the MDE-DH approximation is inadequate in these cases.

From the results from the EDHX approximation we see that apart from dilute systems it is clearly insufficient to have only one decay mode. We need to include at least two decay modes with different decay parameters $\kappa$ and $\kappa^{\prime}$ as shown in eqn (74) and the corresponding expression for $g_{+ \pm}(r)$ mentioned earlier. Then we have at least four parameters to determine $\kappa, \kappa^{\prime}, \mathcal{E}_{\mathrm{r}}^{\text {eff }}$ and $\mathcal{E}_{\mathrm{r}}^{\text {eff }}$. For the monovalent case we have the fortunate circumstance that $\kappa$ and $\kappa^{\prime}$ can be quite accurately be determined by solving eqn (33) and that $\mathcal{E}_{\mathrm{r}}^{\text {eff }}$ and $\mathcal{E}_{\mathrm{r}}^{\text {eff }}$ then can be determined from the application of the local electroneutrality condition and the Stillinger-Lovett second moment condition. This is not the case in general so one will need to apply further conditions that allow four parameters or more to be determined. This will not be pursued in the current paper.

\section{The various types of deviations of $\boldsymbol{\kappa}$ from $\boldsymbol{\kappa}_{\mathrm{D}}$ for symmetric electrolytes}

The deviations of $\kappa$ from $\kappa_{\mathrm{D}}$ can be analyzed in terms of the effective charges $q^{\text {eff }}$ since we have the exact relationship eqn (19), $\kappa^{2}=\beta \sum_{i} n_{i} q_{i} q_{i}^{\text {eff }} /\left(\varepsilon_{\mathrm{r}} \varepsilon_{0}\right)$, which can be written as [eqn (51)]

$$
\left[\frac{\kappa}{\kappa_{\mathrm{D}}}\right]^{2}=\frac{q^{\mathrm{eff}}}{q}
$$

for the RPM. For the monovalent electrolytes in aqueous solution we have seen that $\kappa>\kappa_{\mathrm{D}}$ for most concentrations, so the decay length $1 / \kappa$ is shorter than the Debye length $1 / \kappa_{\mathrm{D}}$ and we have over-screening. The relationship to the effective charge given in eqn (78) implies that $q^{\text {eff }}$ is larger than the actual (bare) ionic charge $q$. This is also apparent from our approximate formula (31) for $q^{\text {eff }}$.

The fact that $q^{\text {eff }}>q$ for most concentrations in the monovalent case is simply a geometrical effect of the finite ion size. When the ion diameter $a$ is increased from some small value, the surrounding ion cloud is pushed further out, as apparent already in the DH approximation, where eqn (11) for $r \geq a$ can be written as

$$
\rho_{i}(r)=-\frac{\kappa_{\mathrm{D}}^{2} q_{i}}{4 \pi\left(1+\kappa_{\mathrm{D}} a\right)} \cdot \frac{\mathrm{e}^{-\kappa_{\mathrm{D}}(r-a)}}{r}
$$


with the factor $(r-a)$ in the exponent. The range of the cloud is extended due to this factor when $a$ is increased. This makes the electrostatic potential larger far from the ion, as apparent in eqn (10) for the $\mathrm{DH}$ case, so the ion appears to have a larger charge, that is, a larger $q^{\text {eff }}$. In the $\mathrm{DH}$ approximation this applies only for the central ion, but in the general case this applies to all ions because all of them are treated in the same manner, so if $q^{\text {eff }}>q$ we have $\kappa>\kappa_{\mathrm{D}}$.

For cases like divalent $(2: 2)$ electrolytes where $\kappa<\kappa_{\mathrm{D}}$, there is, instead under-screening, so the decay length $1 / \kappa$ is longer than the Debye length and $q^{\text {eff }}<q$. This lowering of $q^{\text {eff }}$ is caused by strong anion-cation correlations; each ion strongly attracts ions of opposite charge causing large values of $g_{+-}(r)$ near contact, $r=a$. The large amount of opposite charge near the ion leads to a reduction of the potential for large $r$. The ion thus appear to have a smaller charge, that is, a smaller $q^{\text {eff }}$. The strong (nonlinear) anion-cation correlational attractions hence gives $q^{\text {eff }}<q$ and $\kappa<\kappa_{\mathrm{D}}$. Nothing more than strong anion-cation correlations are needed to give rise to the negative deviations of $\kappa$ from $\kappa_{\mathrm{D}}$ in dilute solutions. This is apparent from the EDHX approximation, which shows this behavior, and when the electrostatic coupling is large, this gives, as we have seen, a large negative deviation.

Decay lengths $1 / \kappa$ that are much longer than the the Debye length $1 / \kappa_{\mathrm{D}}$ have been experimentally obtained in surface force measurements of divalent $(2: 2)$ and trivalent $(3: 3)$ electrolytes in dilute aqueous solutions ${ }^{104}$ and monovalent electrolytes in dilute isopropanol solutions ${ }^{105}$ by Trefalt and coworkers. Isopropanol has dielectric constant $\varepsilon_{\mathrm{r}}=17.9$, so the electrostatic coupling for monovalent ions in isopropanol corresponds approximately to divalent ions in water. Their experimental results are shown in Fig. 12 together with theoretical data that will be discussed later.

They analyzed their results in terms of ion pairing, where anion-cation pairs are in equilibrium with dissociated ions, as described by a simple mass action law with equilibrium constant $K$, where the ionic activity coefficients are set to one. By only including the dissociated (free) ions with density $n_{i}^{\text {free }}$ in the $\mathrm{DH}$ expression (1) for the decay parameter, they obtained an effective $\kappa$, defined from $\left(\kappa_{\mathrm{D}}^{\mathrm{eff}}\right)^{2}=\beta \sum_{i} n_{i}^{\text {free }} q_{i}^{2} /\left(\varepsilon_{\mathrm{r}} \varepsilon_{0}\right)$, which can be written

$$
\left[\frac{\kappa_{\mathrm{D}}^{\mathrm{eff}}}{\kappa_{\mathrm{D}}}\right]^{2}=\frac{n^{\text {free }}}{n}
$$

for symmetric electrolytes. The paired ions thereby do not contribute since the pair has zero net charge. This expression was successfully fitted by Trefalt and coworkers to the measured values of $\kappa$ by selecting reasonable values for $K$. This approach leads to the asymptotic formula ${ }^{104}$

$$
\left[\frac{\kappa_{\mathrm{D}}^{\mathrm{eff}}}{\kappa_{\mathrm{D}}}\right]^{2} \sim 1+a_{1} \Lambda^{2}=1+a_{2}\left(\kappa_{\mathrm{D}} a\right)^{2},
$$

when $\Lambda$ and $\kappa_{\mathrm{D}} a \rightarrow 0$,

where $a_{1}$ is a negative constant that is proportional to $K$ and $a_{2}=a_{1} \beta_{R}^{2} / z^{4}$. This does not agree with the exact formula (73),
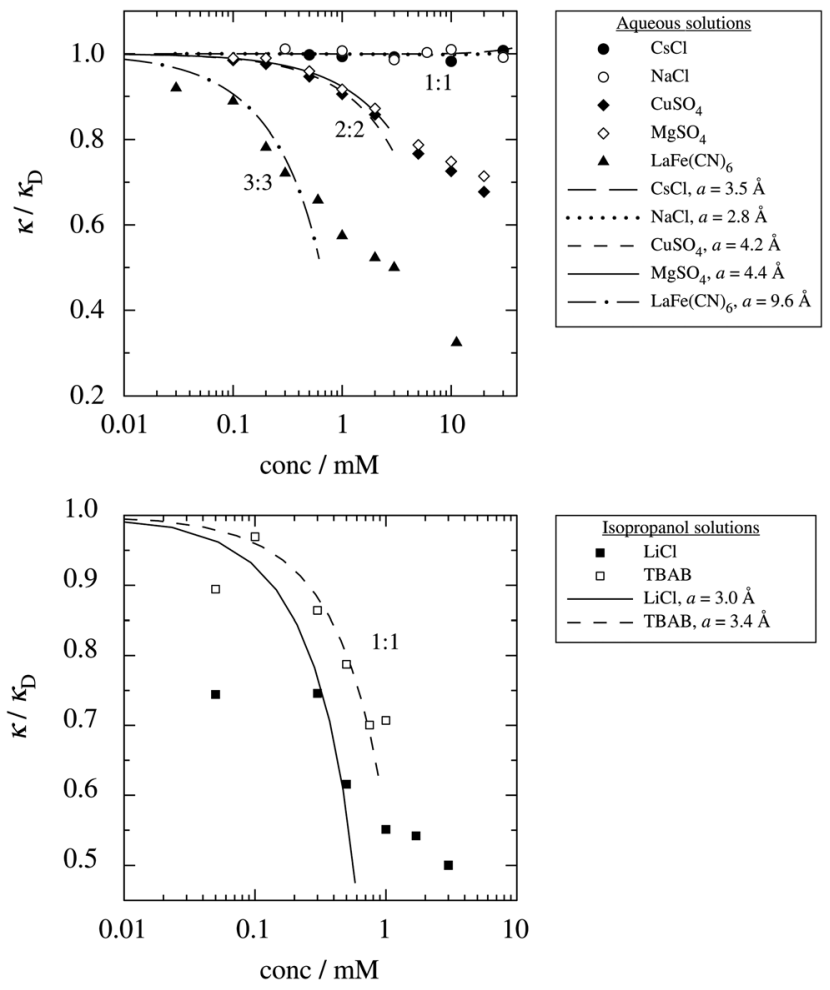

Fig. 12 The ratio $\kappa / \kappa_{\mathrm{D}}$ as function of concentration on a logarithmic scale for monovalent $(1: 1)$, divalent $(2: 2)$ and trivalent $(3: 3)$ electrolytes in dilute aqueous solution and monovalent $(1: 1)$ electrolytes in dilute isopropanol solution. Experimental data from ref. 104 and 105 are shown as symbols and predictions of the asymptotic formula (81) for low concentrations are shown as curves. The values of the ionic diameter $a$, which is the single fitting parameter for the asymptote, are shown in the rectangles to the right in the plot, where also the ionic species are displayed.

but can nevertheless be a fair approximation within a finite concentration interval. Eqn (80) says that the deviation $\left(\kappa_{\mathrm{D}}^{\text {eff }} / \kappa_{\mathrm{D}}\right)^{2}$ from 1 is proportional to the ion concentration in the limit of infinite dilution. As we have seen, this is typical for approximations where nonlinear ion-ion correlations are not correctly included.

Ion pairing in simple electrolytes is an example of strong anion-cation correlations, where the contact value of $g_{+-}(r)$ is very large. The pairing is a transient state for the ions involved in the pairing. Fundamentally, all ions should be treated on the same basis irrespectively if they are paired or not, but as an approximation one can model the system such that paired ions are treated as a separate species as done in the pairing theories mentioned in the Introduction. The effective charge $q^{\text {eff }}$ above for symmetric electrolytes, thereby correspond to an average over free ions and ions that are members of some pair,

$$
q^{\mathrm{eff}}=\frac{n^{\mathrm{free}} q_{\mathrm{free}}^{\mathrm{eff}}+\left(n-n^{\text {free }}\right) q_{\text {paired }}^{\text {eff }}}{n},
$$

where $q_{\text {paired }}^{\text {eff }}=0$ in the RPM. Since the paired species does not contribute to $\kappa^{2}$ given by eqn (19), we obtain

$$
\left[\frac{\kappa}{\kappa_{\mathrm{D}}}\right]^{2}=\frac{n^{\text {free }}}{n} \cdot \frac{q_{\mathrm{free}}^{\mathrm{eff}}}{q} .
$$


In the approach by Trefalt and coworkers, they have accordingly tacitly assumed that the effective charge of the free ions is equal to the bare ionic charge, which gives eqn (79) without the factor $q_{\text {free }}^{\text {eff }} q$. This can be a reasonable approximation for a system with high electrostatic coupling provided $n^{\text {free }} \ll n$. However, this assumption, together with their additional assumption that the ionic activity coefficients are equal to one, set a limit on the applicability of their approach. The equilibrium condition used for paired and free ions thereby constitutes a simple model for the effects of ion-ion correlations. The reason why the asymptotic expression (80) does not agree with the exact formula (73) is the approximation $q_{\text {free }}^{\text {eff }}=q$.

Even if an ion pair is a transient state caused by strong anion-cation correlations rather than a kind of separate species, the two ions will act like a dipole in the interactions with the other ions including other transient pairs. There may also be other transient aggregates with more than two ions. Pairs and other transient aggregetes and their effects on the decay lengths and the effective dielectric permittivities of the electrolyte can be handled by the exact DIT formalism. ${ }^{94,95}$ It is, for example, found that the strong anion-cation attractive correlations at short separations (transient pairs corresponding to high values of $g_{+-}(r)$ near contact) have qualitatively the same effect on the dielectric properties of the electrolyte as an actual pair. As we saw in Fig. 11, we have $\mathcal{E}_{\mathrm{r}}^{\mathrm{eff}}>\varepsilon_{\mathrm{r}}$ for $2: 2$ electrolytes of low to moderate concentrations, so there is an augmented dielectric permittivity of the electrolyte as expected from such a mechanism.

In order to investigate the dilute region further, let us extract the leading asymptotic terms for $\kappa^{2} / \kappa_{\mathrm{D}}^{2}$ and $\mathcal{E}_{\mathrm{r}}^{\text {eff }} / \varepsilon_{\mathrm{r}}$ in the limit $\tau=\kappa_{\mathrm{D}} a \rightarrow 0$ from eqn (76) and (77) of the EDHX approximation. We will thereby include terms up to order $\tau^{2}$, that is, up to terms that are proportional to concentration. Since this approximation is designed to give accurate results for dilute electrolytes, the derived asymptotic formulas should be correct in the limit of zero concentration. As shown in Appendix D we obtain

$$
\begin{aligned}
{\left[\frac{\kappa}{\kappa_{\mathrm{D}}}\right]^{2} \sim } & 1+\frac{\beta_{R}^{2}}{6} \tau^{2} \ln \tau \\
& +\left[\frac{1}{2}+\frac{\beta_{R}^{2} \mathcal{D}}{6}-\beta_{R}^{2} s\left(\beta_{R}^{2}\right)\right] \tau^{2} \quad \text { when } \tau=\kappa_{\mathrm{D}} a \rightarrow 0
\end{aligned}
$$

and

$$
\left[\frac{\mathcal{E}_{\mathrm{r}}^{\mathrm{eff}}}{\varepsilon_{\mathrm{r}}}\right]^{-1} \sim 1-\frac{\beta_{R}^{2}}{324} \tau^{2} \quad \text { when } \tau=\kappa_{\mathrm{D}} a \rightarrow 0
$$

where the constant $\mathcal{D}=\mathcal{C}_{\mathrm{Eu}}+\ln 3+\frac{1}{54} \approx 1.6944, \mathcal{C}_{\mathrm{Eu}} \approx 0.5772$ is Euler's constant and $s(x)=x /(5 ! \cdot 2)+x^{2} /(7 ! \cdot 4)+\ldots$ is defined in eqn (136) of Appendix D.

In the rhs of eqn (81), the second term, which is negative for small $\kappa_{\mathrm{D}} a$, dominates when $\kappa_{\mathrm{D}} a \rightarrow 0$ due to the logarithm and decays to zero in this limit. Eqn (81) agrees with the exact limiting law (73) since $\beta_{R} \tau=z^{2} \Lambda$ and $\ln \tau=\ln (\Lambda)+\ln \left(z^{2} / \beta_{R}\right) \sim$ $\ln (\Lambda)$ when the concentration and hence $\Lambda$ and $\tau$ go to zero.

For divalent electrolytes in aqueous solution, the predictions of the asymptotic formulas (81) and (82) are plotted as red dotted curves in Fig. 11a and b, respectively. Despite that these formulas were derived from the EDHX approximation in the limit zero concentrations, each asymptote continues not far from the simulated results in a quite wide concentration interval and give therefore surprisingly reasonable estimates of the latter. This means that the asymptotes can be useful also outside the region of very high dilution, at least for the purpose of approximate assessments of ion-ion correlation effects in $\kappa^{2} / \kappa_{\mathrm{D}}{ }^{2}$ and $\mathcal{E}_{\mathrm{r}}^{\text {eff }} / \varepsilon_{\mathrm{r}}$ at high electrostatic coupling where the MDE$\mathrm{DH}$ approximation is inadequate.

As a test of such an assessment, the asymptote from formula (81) has been compared with the experimental data by Trefalt an coworkers ${ }^{104,105}$ mentioned earlier. || || || Data are available for $\mathrm{CsCl}, \mathrm{NaCl}, \mathrm{CuSO}_{4}, \mathrm{MgSO}_{4}$ and $\mathrm{LaFe}(\mathrm{CN})_{6}$ solutions in water and tetrabutylammonium bromide (TBAB) and $\mathrm{LiCl}$ solutions in isopropanol. Note that for a given $T, \varepsilon_{\mathrm{r}}$ and valency $z$ of the symmetric electrolyte, the predicted asymptote as function of concentration contains only one parameter, namely the ionic diameter $a$. In the asymptotic formula, the diameter will correspond to an average value since the anions and cations in the experiments have somewhat different sizes.

The results of a comparison with the experimental data with $a$ as the single fitting parameter is presented in Fig. 12, whereby all cases apart from monovalent ions in water ( $\mathrm{CsCl}$ and $\mathrm{NaCl}$ ) were fitted; the experimental uncertainties for the latter were too large to make a fit meaningful. For the other cases, the fits to the low-concentration part of the experimental data are quite good overall considering the experimental uncertainties. The values of the ionic diameters obtained are reasonable, but as we will see below, they are probably somewhat too large. Note that ionic sizes used in primitive model calculations fitted to experimental data are usually different from bare diameters and often include effects of hydration, in particular for multivalent ions. As regards the $\mathrm{LiCl}$ solutions in isopropanol there are to few experimental data points available in order to say something definite about the fit in this case. The first data point to the left in the plot for this system and for TBAB in isopropanol are outliers, which demonstrate the experimental difficulties in this kind of measurements.

We can conclude that strong anion-cation correlations constitute the explanation for the experimentally observed over-screening in dilute solutions at room temperature when $z$ is high or $\varepsilon_{\mathrm{r}}$ is low, whereby the decay lengths are considerably

Фबा Note that $1+\beta_{R}{ }^{2} \tau^{2} \ln (\tau) / 6=1+z^{4} \Lambda^{2} \ln \left(\kappa_{\mathrm{D}} a\right) / 6$ contains the hard core diameter $a$ and is not a universal limiting law for $\kappa^{2} / \kappa_{\mathrm{D}}^{2}$ valid for all symmetric electrolytes because it presumes that the ions are hard spheres. The universal law (73) instead contains the factor $\ln \left(\kappa_{\mathrm{D}} \ell_{\mathrm{B}}\right)$.

|| || A favorable comparison between the experimental data and the exact asymptotic expression (73) was done in ref. 104 for the aqueous solutions. Such a comparison could not be done for the isopropanol solutions for reasons outlined in footnote $+\uparrow+$, but eqn (81) can be used for both kinds of solutions since it is expressed solely in terms of $\tau$ and $\beta_{R}$. 

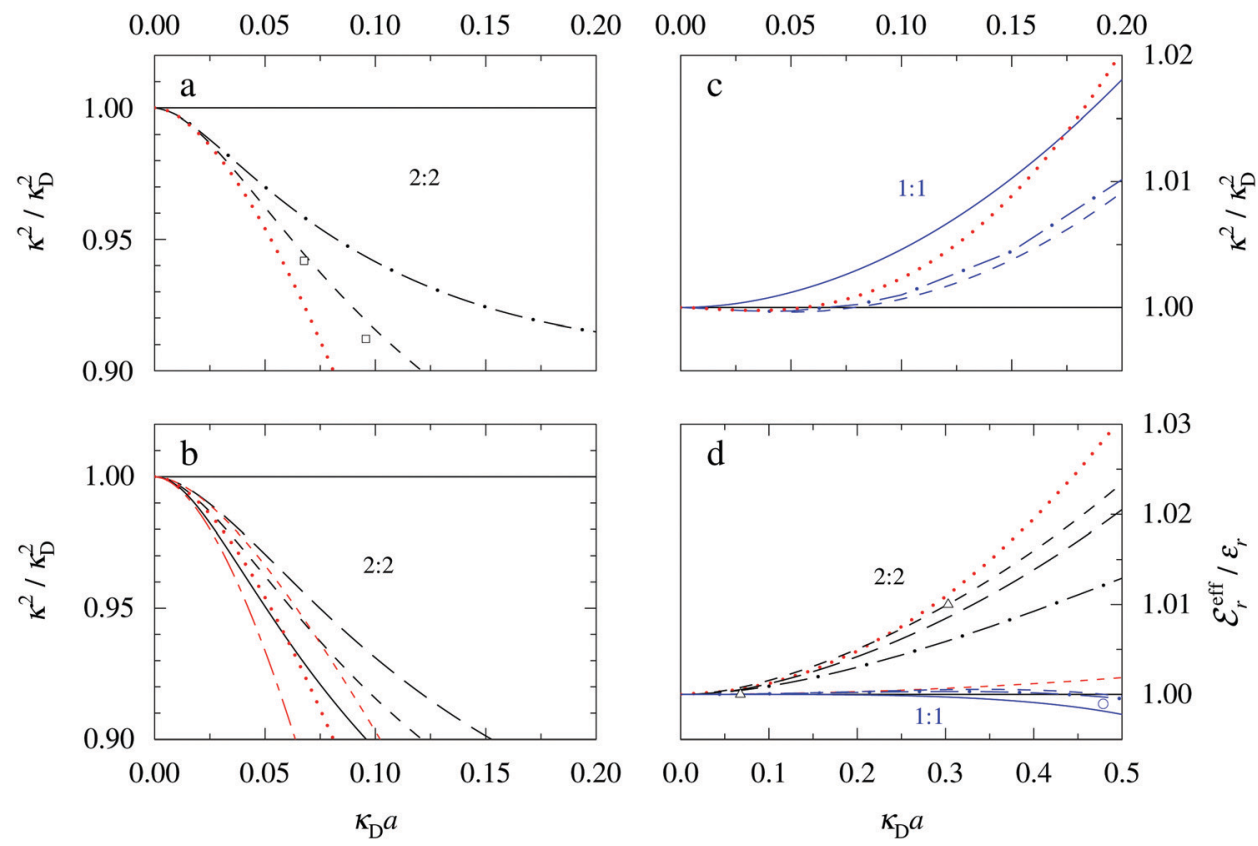

Fig. 13 The ratios $\kappa^{2} / \kappa_{\mathrm{D}}^{2}$ and $\mathcal{E}_{\mathrm{r}}^{\text {eff }} / \varepsilon_{\mathrm{r}}$ as functions of $\kappa_{\mathrm{D}}$ a for divalent $(2: 2)$ and monovalent $(1: 1)$ electrolytes in dilute aqueous solution compared with the asymptotes (red curves). (a) The same curves as in Fig. 11a for the divalent case shown the dilute region at large magnification. The red dotted curve is the asymptote for $\kappa^{2} / \kappa_{\mathrm{D}}^{2}$ from eqn (81). The ion diameter is $a=4.6 \AA$ and $\beta_{R}=6.22$. (b) An illustration of the ion size dependence of $\kappa^{2} / \kappa_{\mathrm{D}}^{2}$ for divalent electrolytes; from bottom to top $a=4.2,4.6$ and 5.0 $\AA$. Black curves are from HNC calculations and red curves from the asymptotic formula (81). (c) The red dotted curve shows the asymptote for $\kappa^{2} / \kappa_{\mathrm{D}}^{2}$ from eqn (81) for monovalent electrolytes with $\beta_{R}=1.55$. The full, dashed-dotted and dashed curves (MDE-DH, EDHX and HNC results respectively) are the same as the corresponding curves in Fig. 10a. (d) $\mathcal{E}_{\mathrm{r}}^{\text {eff }} / \varepsilon_{\mathrm{r}}$ for divalent electrolytes (black curves and red dots, $\beta_{R}=6.22$ ) and monovalent electrolytes (blue curves and red mini-dashes, $\beta_{R}=1.55$ ) in aqueous solution. The red curves show $\mathcal{E}_{\mathrm{r}}^{\text {eff }} / \varepsilon_{\mathrm{r}}$ from the asymptotic expression (82). The curves for the divalent case are the same as in Fig. 11b shown the dilute region at large magnification and the blue curves are the same as the corresponding ones in Fig. 10b. The HNC and MC data are taken from ref. 18 and 22.

longer than the Debye length. The same is true for thin ionic plasmas at moderately high temperatures. There is no need to assume actual ion pairing, although such pairing can be used as a simple way to model such correlations as mentioned in the Introduction and discussed above. The asymptotic formula (81) can accordingly be used as a simple means to approximately assess nonlinear ion-ion correlations effects in dilute solutions. The fact that the asymptote nearly quantitatively describes the variation of the decay length with electrostatic coupling strengths (varying $z, \varepsilon_{\mathrm{r}}$ or $T$ ) is thereby a great advantage.

Finally, it is of interest to investigate the asymptotes from eqn (81) and (82) in more detail in the dilute region. The ratios $\kappa^{2} / \kappa_{\mathrm{D}}^{2}$ and $\mathcal{E}_{\mathrm{r}}^{\text {eff }} / \varepsilon_{\mathrm{r}}$ for divalent and monovalent symmetric electrolytes in water are plotted in Fig. 13 as functions of $\kappa_{\mathrm{D}} a$ for low values of the latter. Fig. 13a shows a magnified view of Fig. 11a in the dilute region of the $2: 2$ case and we see that the asymptote joins the other curves when $\kappa_{\mathrm{D}} a \rightarrow 0$ as it should. It thereby joins the HNC curve somewhat faster than the curve from the EDHX approximation in this limit.

Fig. 13b shows how the ion diameter affects both the HNC curves and the asymptotes when $a$ varies between 4.2 to $5.0 \AA$ for $2: 2$ electrolytes. The middle case, $a=4.6 \AA$, is the same as in frame (a) of the figure. We can see that the asymptote for a certain $a$ value, say $a=5.0 \AA$, lies close to the HNC curve for a slightly smaller $a$ value, say $4.6 \AA$, in a whole $\kappa_{\mathrm{D}} a$ interval.
This implies that the fits made in Fig. 12 for the asymptotes most likely give somewhat too large values of $a$.

In Fig. 13c it is seen that the asymptote for the monovalent case accurately describes the slight dip of $\kappa^{2} / \kappa_{\mathrm{D}}^{2}$ below 1 for small $\kappa_{\mathrm{D}} a$. When $\kappa_{\mathrm{D}} a$ is increased, the asymptote approaches and then crosses the full curve, that is, the decay parameter from the MDE-DH approximation, eqn (33), which goes like like $\kappa^{2} / \kappa_{\mathrm{D}}^{2} \sim 1+\tau^{2} / 2$ for small $\tau$. We may note that eqn (81) contains a $\tau^{2} / 2$ contribution, namely from the term $1 / 2$ in the coefficient of the $\tau^{2}$ term.

The ratio $\mathcal{E}_{\mathrm{r}}^{\text {eff }} / \varepsilon_{\mathrm{r}}$ for both divalent and monovalent electrolytes is plotted in Fig. 13d. For the 2:2 case the asymptote lies quite closely to the the accurate results in a wide interval, as we saw previously in Fig. 11. The asymptote in the $1: 1$ case joins the accurate curves from above, $\mathcal{E}_{\mathrm{r}}^{\mathrm{eff}} / \varepsilon_{\mathrm{r}}>1$, in the dilute region, where these latter curves lie slightly above 1 , as seen more clearly in the inset to Fig. 10b.

\section{Summary and concluding remarks}

This work is based on the following observations. In electrolytes there are several decay modes for the electrostatic interactions, each having a decay length and a magnitude. In the DH and PB approximations there is, however, only one mode that gives a screened Coulomb potential equal to $\phi_{\text {Coul }^{*}}(r)=\mathrm{e}^{-\kappa_{\mathrm{D}} r} /\left(4 \pi \varepsilon_{0} \varepsilon_{\mathrm{r}} r\right)$ 
with a decay length equal to the Debye length $1 / \kappa_{\mathrm{D}}$ and magnitude proportional to $1 / \varepsilon_{\mathrm{r}}$. The mean electrostatic potential $\psi_{i}(r)$ due to an ion of species $i$ is in the DH approxi-

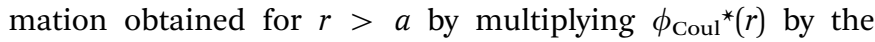
effective charge $q_{i}^{\text {eff }}$ given by eqn (12), so we have $\psi_{i}(r)=$ $q_{i}^{\text {eff }} \mathrm{e}^{-\kappa_{\mathrm{D}} r} /\left(4 \pi \varepsilon_{0} \varepsilon_{\mathrm{r}} r\right)$. In the PB approximation $\psi_{i}(r)$ decays for large $r$ as the rhs of this expression.

A correct treatment of electrolytes provides a screened Coulomb potential $\phi_{\text {Coul }}{ }^{*}(r)$ with multiple decay modes, where the modes give contributions like ( $c f$. eqn (23))

$$
\text { contributions in } 4 \pi \varepsilon_{0} \phi_{\text {Coul }}{ }^{*}(r): \frac{\mathrm{e}^{-\kappa r}}{\mathcal{E}_{\mathrm{r}}^{\text {eff }} r} \text { and } \frac{\mathrm{e}^{-\kappa^{\prime} r}}{\mathcal{E}_{\mathrm{r}}^{\prime \text { eff }} r} \text {. }
$$

with magnitudes inversely proportional to $\mathcal{E}_{\mathrm{r}}^{\text {eff }}$ and $\mathcal{E}_{\mathrm{r}}^{\text {eff }}$, respectively, which are effective dielectric permittivities of the electrolyte for the respective mode. They depend on the dielectric response of the electrolyte. We have here only included the two main modes with the largest decay lengths. The mean electrostatic potential $\psi_{i}(r)$ has the following principal contributions from these modes ( $c f$. eqn (25))

$$
\text { contributions in } 4 \pi \varepsilon_{0} \psi_{i}(r): \frac{q_{i}^{\text {eff }} \mathrm{e}^{-\kappa r}}{\mathcal{E}_{\mathrm{r}}^{\mathrm{eff}} r} \text { and } \frac{q_{i}^{\prime \text { eff }} \mathrm{e}^{-\kappa^{\prime} r}}{\mathcal{E}_{\mathrm{r}}^{\prime} \text { eff } r} \text {. }
$$

where $q_{i}^{\text {eff }}$ and $q_{i}^{\text {eff }}$ are the effective charges of the $i$-ion, whereby each mode has its own value of this charge. The decay parameter $\kappa$ can be expressed in terms of $q_{j}^{\text {eff }}$ as $\kappa^{2}=$ $\beta \sum_{j} n_{j} q_{j} q_{j}^{\text {eff }} /\left(\varepsilon_{\mathrm{r}} \varepsilon_{0}\right)$ [eqn (4) and (24)] and $\kappa^{\prime}$ is given by the analogous expression with $q_{i}^{\text {eff }}$. The electrostatic part of the potential of mean force $w_{i j}(r)$ for species $i$ and $j$ has contributions with yet another factor of effective charge, namely (cf. eqn (21))

$$
\text { contributions in } 4 \pi \varepsilon_{0} w_{i j}^{\mathrm{el}}(r): \frac{q_{i}^{\mathrm{eff}} q_{j}^{\mathrm{eff}} \mathrm{e}^{-\kappa r}}{\mathcal{E}_{\mathrm{r}}^{\mathrm{eff}} r} \text { and } \frac{q_{i}^{\prime \text { eff }} q_{j}^{\prime \text { eff }} \mathrm{e}^{-\kappa^{\prime} r}}{\mathcal{E}_{\mathrm{r}}^{\prime \mathrm{eff}} r} \text {. }
$$

For elevated ionic densities these functions turn into exponentially damped oscillatory ones, whereby $\kappa$ and $\kappa^{\prime}$ are complexvalued, $\kappa=\kappa_{\Re}+\mathrm{i} \kappa_{\mathfrak{I}}$ and $\kappa^{\prime}=\kappa_{\Re}-\mathrm{i} \kappa_{\mathfrak{I}}$. The contribution in eqn (85) from the second mode then is the complex conjugate of that from the first. The sum of the two modes then decays like $\mathrm{e}^{-\kappa_{\Re} r} \cos \left(\kappa_{\Im} r+\alpha\right)$, where $\alpha$ is a phase.

In the Multiple-Decay Extended Debye-Hückel, MDE-DH, approximation developed in the current work, one assumes as an approximation that solely the first two decay modes contribute to the electrostatics in electrolytes, so $w_{i j}^{\text {el }}$ is given by the sum of the contributions in eqn (85). Furthermore one takes $w_{i j}(r)=w_{i j}^{\text {el }}(r)+w_{i j}^{\text {core }}(r)$, where $w_{i j}^{\text {core }}$ is the contribution from the ionic core-core correlations that is approximated by $w^{\text {hs }}$ from a hard sphere fluid. The term $w_{i j}^{\text {core }}$ expresses a further decay mode for the interactions. We have

$$
g_{i j}(r)=\mathrm{e}^{-\beta\left[w_{i j}^{\mathrm{el}}(r)+w_{i j}^{\mathrm{core}}(r)\right]}=\left[1-\beta w_{i j}^{\mathrm{el}}(r)+\left[\beta w_{i j}^{\mathrm{el}}(r)\right]^{2} / 2+\ldots\right] \mathrm{e}^{-\beta w_{i j}^{\mathrm{core}}(r)}
$$

and only the first few terms of the expansion in $\beta w_{i j}^{\text {el }}$ are used as a further approximation, so the MDE-DH approximation is limited to sufficiently weak electrostatic coupling where $\beta w_{i j}^{\mathrm{el}}$ is small. The decay parameters $\kappa$ and $\kappa^{\prime}$ used are solutions to the equation $\kappa^{2} / \kappa_{\mathrm{D}}^{2}=\mathrm{e}^{\kappa a} /(1+a \kappa)$ [see eqn (33)] originally derived in the LT and $\mathrm{MDH}$ approximations. ${ }^{32,33}$ These solutions are presented in Fig. 2. The effective charges are obtained by inserting the respective $\kappa$ value into $q_{i}^{\text {eff }}=q_{i} \mathrm{e}^{\kappa a} /(1+\kappa a)$ [eqn (31)]. By applying the necessary local electroneutrality and the Stillinger-Lovett second moment conditions, explicit expressions for $\mathcal{E}_{\mathrm{r}}^{\text {eff }}$ and $\mathcal{E}_{\mathrm{r}}^{\text {eff }}$ are derived and the resulting theory does not contain any fitting parameters.

For symmetric electrolytes with ions of diameter $a$, which is the main case treated in the current work, $\psi_{i}(r)$ is proportional to the ionic charge $q_{i}$, so the MDE-DH theory is a linearized approximation for the electrostatic response like, for example, the MSA, GMSA, LMPB, MDH and GDH theories. In contrast to these latter approximations, the MDE-DH theory contains the square term $\left[\beta w_{i j}^{\mathrm{el}}(r)\right]^{2} / 2$, which contributes to the pair distributions but not to $\psi_{i}$ (see Section 4.2). This term guarantees that $g_{i j}(r) \geq 0$ for all $r$ and leads to a good thermodynamic consistency.

The approximation comes in two versions, the Simple and the Complete MDE-DH approximations (the differences between the two are explained in Section 4.2, eqn (55) ff.). The simple version is applicable when $w_{i j}^{\text {core }}$ is not too large, for example systems with small ions. Its results are in very good agreement with simulations and Hypernetted Chain calculations for all concentrations investigated, including high ones, provided the electrostatic coupling is not too high for instance monovalent electrolytes in water at room temperature. All numerical results in Section 4 apart from Fig. 7, where the ions are large, are calculated in this approximation. Furthermore, in the Simple MDE-DH approximation, there are simple analytical expressions for $\mathcal{E}_{\mathrm{r}}^{\text {eff }}$ and $\mathcal{E}_{\mathrm{r}}^{\text {eff }}$ [eqn (46) and (47)] and for the thermodynamic properties: the activity coefficient $\gamma_{ \pm}$is given by $\ln \gamma_{ \pm}=\ln \gamma_{ \pm}^{\text {el }}+\ln \gamma_{ \pm}^{\text {core }}$ with $\ln \gamma_{ \pm}^{\text {el }}$ from eqn (64) and $\ln \gamma_{ \pm}^{\text {core }}$ from eqn (60) and the osmotic coefficient $\phi=1+\phi^{\text {el }}+\phi^{\text {cont }}$ with $\phi^{\text {el }}$ from eqn (69) and $\phi^{\text {cont }}$ from eqn (68) [see also eqn (124)]. These expressions are valid also for complex-valued $\kappa$ and $\kappa^{\prime}$, but they are reformulated in Appendix $\mathrm{C}$ into equivalent formulas that are more easy to use for that case.

The Complete MDE-DH approximation is used to obtain the results in Fig. 7, which shows the pair distribution functions for a dense system with large monovalent ions. These results are in excellent agreement with simulations, including quite intricate details. It is, however, sufficient to use the formulas of Simple MDE-DH approximation to calculate the thermodynamic quantities for this case; the difference between the two versions of the approximation for these quantities is less than $1 \%$. Thus, in practical applications, the simple version of the approximation is in many cases sufficient unless structural entities are required. This is agreement with the observation discussed in the Introduction that it is simpler to calculate thermodynamical entities than structural ones. 
In the MDE-DH approximations and the other approximations with linearized electrostatic response mentioned above, we have $\kappa \geq \kappa_{\mathrm{D}}$ for all concentration where $\kappa$ is real, while the exact limiting law (5) says that there is under-screening, $\kappa<\kappa_{\mathrm{D}}$, for sufficiently dilute solutions of symmetric electrolytes. This under-screening is caused by nonlinear electrostatic response of the electrolyte that is not included in the MDE-DH approximations. In order to investigate this matter, the nonlinear approximation given by the first equality in eqn (86) is used in the low density limit, where contributions from $w_{i j}^{\text {core }}$ and the second decay mode in $w_{i j}^{\text {el }}$ go to zero and therefore can be neglected. The approximation where these contributions to $w_{i j}$ are not included is called the Extended DHX, EDHX, approximation since it is an extension of the exponential Debye-Hückel, DHX, approximation ( $c f$. Section 2).

The EDHX approximation is used to calculate $\kappa$ and $\mathcal{E}_{\mathrm{r}}^{\text {eff }}$ for symmetric electrolytes and it is found that $\kappa<\kappa_{\mathrm{D}}$ for sufficiently dilute solutions in agreement with the exact limiting law. Furthermore, it is found that $\mathcal{E}_{\mathrm{r}}^{\text {eff }}>\varepsilon_{\mathrm{r}}$ in the dilute region, while the linear approximations predict that $\mathcal{E}_{\mathrm{r}}^{\mathrm{eff}}<\varepsilon_{\mathrm{r}}$. The EDHX results are thereby in agreement with the HNC approximation, which is considerably more complex. In the case of monovalent electrolytes in water at room temperature, it is found that this qualitatively incorrect behavior of the linear approximations has negligible consequences in practice because they occur for so low concentrations that the deviations of $\kappa / \kappa_{\mathrm{D}}$ and $\mathcal{E}_{\mathrm{r}}^{\mathrm{eff}} / \varepsilon_{\mathrm{r}}$ from 1 are miniscule.

For multivalent ions in water and monovalent ions in solvents with considerable lower $\varepsilon_{\mathrm{r}}$, the situation is completely different and under-screening is very substantial for dilute solutions of symmetric electrolytes, as found by MC simulations, HNC calculations and experiments. Likewise, $\mathcal{E}_{\mathrm{r}}^{\text {eff }}$ is considerably larger than $\varepsilon_{\mathrm{r}}$ for the dilute solutions due to the nonlinear ionion correlation effects. An approximate limiting law (81) for symmetric electrolytes that extends the exact law by including higher order terms is derived in the EDHX approximation and a corresponding law for $\mathcal{E}_{\mathrm{r}}^{\text {eff }} / \varepsilon_{\mathrm{r}}$, eqn (82), is also derived. These approximate laws contain the ion size dependence of $\kappa$ and $\mathcal{E}_{\mathrm{r}}^{\text {eff }}$ for dilute solutions. Despite that they are strictly valid only for very low concentrations, they can be used as a reasonable approximation in a wider concentration interval to describe the effect of nonlinear ion-ion correlation effects in dilute electrolytes. The approximate limiting law for $\kappa / \kappa_{\mathrm{D}}$ is used to analyze the experimental results by Trefalt and coworkers ${ }^{104,105}$ for this ratio in aqueous multivalent electrolyte solutions and monovalent electrolytes dissolved in isopropanol. The law is successfully fitted to the experimental results for low concentrations whereby the ion diameter is the sole fitting parameter. This gives important insights into the reasons behind the deviations of $\kappa$ from $\kappa_{\mathrm{D}}$ and mechanisms of the latter is discussed in some detail Section 6.

In order to give a further perspective on the decay modes and the associated entities in electrolytes, it is worthwhile to mention the following facts regarding more sophisticated models for electrolytes than the primitive model. Recall that for a classical plasma of spherical ions, the decay parameters $\kappa$, $\kappa^{\prime}$ etc. satisfy the exact eqn (24) with $\varepsilon_{\mathrm{r}}=1$. For an electrolyte solution with molecular solvent, that is, in presence discrete polar molecules, we still have $\varepsilon_{\mathrm{r}}=1$ because the particles are in vacuum, but eqn (24) is not valid as it stands because it is, in fact, restricted to systems where all particles are spherically symmetric. The solvent molecules and any ion species that is not spherical give rise to additional terms in this equation that depend on the orientational degrees of freedom of these particles. The equation thereby is replaced by the following exact equation $^{98}$

$$
\begin{aligned}
\kappa^{2}= & \frac{\beta}{\varepsilon_{0}} \sum_{j \in \text { sphercial }} n_{j} q_{j} q_{j}^{\text {eff }}(\kappa) \\
& +\frac{\beta}{\varepsilon_{0}} \sum_{j \in \text { non spherical }} n_{j}\left\langle Q_{j}(\kappa) Q_{j}^{\text {eff }}(\kappa)\right\rangle_{\text {orientations }},
\end{aligned}
$$

where the first sum is over all spherical species and the second sum over the nonspherical ones. Here, $Q_{j}$ is a particleorientation dependent entity that originates from the internal charge distribution of a $j$-particle and $Q_{j}^{\text {eff }}$ is also orientation dependent but originates from both the particle and its surrounding distribution of charge. The entity $Q_{j}^{\text {eff }}$ plays a similar role as a prefactor for $\psi_{i}$ for a nonspherical particle as $q_{j}^{\text {eff }}$ does for a spherical one (for definitions and further details see ref. 98). The average $\langle\cdot\rangle$ in eqn (87) is taken over all orientations of the $j$-particle. The last term contains, for example, effects of solvent-solvent correlations (both orientational and spatial ones). The solvent-ion correlations also contribute to this term and such correlations also influence the values of $q_{j}^{\text {eff }}$ in the presence of solvent because oriented solvent molecules around an ion affects the charge distribution there.

Eqn (87) has a rather complicated form. It is, however, mathematically equivalent to a much simpler, exact equation ${ }^{95,98}$

$$
\kappa^{2}=\frac{\beta}{\mathcal{E}_{\mathrm{r}}{ }^{*}(\kappa) \varepsilon_{0}} \sum_{j} n_{j} q_{j} q_{j}^{*},
$$

where $q_{j}^{*}$ is a renormalized ionic charge ${ }^{* * * *}$ that is different from $q_{j}^{\text {eff }} . \mathcal{E}_{\mathrm{r}}^{*}(\kappa)$, called the dielectric factor, is a kind of renormalized dielectric permittivity, which is not the same as $\mathcal{E}_{\mathrm{r}}^{\mathrm{eff}}(\kappa)$. Both $\mathcal{E}_{\mathrm{r}}{ }^{*}$ and $\mathcal{E}_{\mathrm{r}}^{\text {eff }}$ can be defined in terms of static dielectric response function of the electrolyte. ${ }^{95,98}$ Note that $\mathcal{E}_{\mathrm{r}}{ }^{*}(\kappa)$ is a function of $\kappa$, while $q_{j}^{*}$ is independent of $\kappa$ and has the same value for all decay modes. The decay parameters $\kappa, \kappa^{\prime}$ etc. are solutions to this equation and, of course, also solutions to the equivalent eqn (87). $+1 \dagger \dagger \mathcal{E}_{\mathrm{r}}^{*}(\kappa)$ contains the major effects of solventsolvent, solvent-ion and ion-ion correlations that are important for the decay mode with decay parameter $\kappa$. The value of $q_{j}^{*}$ is, of course, also affected by these correlations, but since it does not depend on $\kappa$, it is not specific for any mode. The general eqn (88)

\footnotetext{
**** The notation used here differs from that in ref. 22, 23, 100 and 101, where $q_{j}^{*}$ denotes the effective charge $q_{j}^{\text {eff }}$ of the present work.

$+\dagger+\dagger$ Both eqn (87) and (88) are mathematically equivalent to the equation $\tilde{\varepsilon}(\mathrm{i} \kappa)=0, c f$. footnote $\neq$ in Section 2 .
} 
has a form that is very similar to the definition (1) of the Debye parameter.

Thus there are two different entities $\mathcal{E}_{\mathrm{r}}{ }^{*}(\kappa)$ and $\mathcal{E}_{\mathrm{r}}^{\mathrm{eff}}(\kappa)$ that take the role that $\varepsilon_{\mathrm{r}}$ has in the $\mathrm{PB}$ and $\mathrm{DH}$ approximations. $\mathcal{E}_{\mathrm{r}}{ }^{*}$ appears in the eqn (88) for $\kappa$ and $\mathcal{E}_{\mathrm{r}}^{\text {eff }}$ appears, as we have seen, in the magnitude of, for example, the screened Coulomb potential $\phi_{\text {Coul }^{*}}$, the mean electrostatic potential $\psi_{j}$ and the charge density $\rho_{j}$. Each mode has its own values for these entities.

In some $\mathrm{PB}, \mathrm{DH}$ and other primitive model approaches one replaces $\varepsilon_{\mathrm{r}}$ by an ion-concentration dependent entity $\varepsilon_{\mathrm{r}}^{\text {electrolyte }}$, which is obtained from macroscopic measurements of the dynamic dielectric response of bulk electrolytes at finite but small frequency, whereby a zero frequency macroscopic response is extracted. This entity contains contributions from the orientational and translational degrees of freedom of the solvent molecules and from ion-solvent correlations that are not included in the primitive model. It is, however, not the same as $\mathcal{E}_{\mathrm{r}}^{*}(\kappa)$ or $\mathcal{E}_{\mathrm{r}}^{\text {eff }}(\kappa)$. When some mode has a small $\kappa$ it may, however, be a reasonable approximation to set $\mathcal{E}_{\mathrm{r}}^{*}(\kappa)$ and $\mathcal{E}_{\mathrm{r}}^{\mathrm{eff}}(\kappa)$ equal to $\varepsilon_{\mathrm{r}}^{\text {electrolyte }}$ for that particular mode, but not for the other modes. When the ion concentration goes to zero, $\mathcal{E}_{\mathrm{r}}^{*} \rightarrow \varepsilon_{\mathrm{r}}$ and $\mathcal{E}_{\mathrm{r}}^{\mathrm{eff}} \rightarrow \varepsilon_{\mathrm{r}}$ for the mode with longest decay length, which goes to the Debye length in this limit.

For a system with spherical particles, eqn (88) is mathematically equivalent to eqn (24). In the primitive model both $\mathcal{E}_{\mathrm{r}}{ }^{*}(\kappa)$ and $\mathcal{E}_{\mathrm{r}}^{\mathrm{eff}}(\kappa)$ solely contains contributions from the ionic degrees of freedom.

\section{Conflicts of interest}

There are no conflicts to declare.

\section{Appendices}

\section{A Pair distribution functions for RPM, theoretical considerations}

In this Appendix we will fill in the details regarding the pair distribution functions for RPM electrolytes in the MDE-DH approximation. As customary, we define the functions

$$
\begin{gathered}
g_{\mathrm{S}}(r)=\frac{1}{2}\left[g_{++}(r)+g_{+-}(r)\right]=\frac{1}{2}\left[g_{--}(r)+g_{+-}(r)\right] \\
g_{\mathrm{D}}(r)=\frac{1}{2}\left[g_{++}(r)-g_{+-}(r)\right]=\frac{1}{2}\left[g_{--}(r)-g_{+-}(r)\right],
\end{gathered}
$$

whereby $g_{+ \pm}(r)=g_{\mathrm{S}}(r) \pm g_{\mathrm{D}}(r)$. Eqn (53) implies that $g_{\mathrm{S}}(r)=$ $\cosh \left[\beta w^{\text {el }}(r)\right] g^{\text {core }}(r)$ and $g_{\mathrm{D}}(r)=-\sinh \left[\beta w^{\mathrm{el}}(r)\right] g^{\operatorname{core}}(r)$, so by inserting eqn (54) we have

$$
g_{\mathrm{S}}(r)=\cosh \left[\frac{\beta\left(q^{\mathrm{eff}}\right)^{2}}{4 \pi \mathcal{E}_{\mathrm{r}}^{\mathrm{eff}} \varepsilon_{0}} \cdot \frac{\mathrm{e}^{-\kappa r}}{r}+\frac{\beta\left(q^{\prime \text { eff }}\right)^{2}}{4 \pi \mathcal{E}_{\mathrm{r}}^{\prime}{ }^{\mathrm{eff}} \varepsilon_{0}} \cdot \frac{\mathrm{e}^{-\kappa^{\prime} r}}{r}\right] g^{\text {core }}(r),
$$

and

$$
g_{\mathrm{D}}(r)=-\sinh \left[\frac{\beta\left(q^{\mathrm{eff}}\right)^{2}}{4 \pi \mathcal{E}_{\mathrm{r}}^{\mathrm{eff}} \varepsilon_{0}} \cdot \frac{\mathrm{e}^{-\kappa r}}{r}+\frac{\beta\left(q^{\prime} \mathrm{eff}^{2}\right.}{4 \pi \mathcal{E}_{\mathrm{r}}^{\prime}{ }^{\prime \text { eff }} \varepsilon_{0}} \cdot \frac{\mathrm{e}^{-\kappa^{\prime} r}}{r}\right] g^{\operatorname{core}}(r),
$$

Note that

$$
\rho(r)=q n 2 g_{\mathrm{D}}(r)=q n_{\mathrm{tot}} g_{\mathrm{D}}(r)
$$

and that the density of ions around an ion is given by $n_{\mathrm{tot}} g_{\mathrm{S}}(r)$. The Debye parameter is given by $\kappa_{\mathrm{D}}{ }^{2}=\beta n_{\text {tot }} q^{2} / \varepsilon_{\mathrm{r}} \varepsilon_{0}$ and we have $\kappa^{2}=\beta n_{\text {tot }} q q^{\text {eff }} / \varepsilon_{\mathrm{r}} \varepsilon_{0}$.

When the electrostatic coupling is sufficiently weak, we can use the approximations $\cosh (x) \approx 1+x^{2} / 2$ and $\sinh (x) \approx x$ in these expressions and obtain as a good approximation

$$
\begin{gathered}
g_{\mathrm{S}}(r)=\left\{1+\frac{1}{2}\left[\beta w^{\mathrm{el}}(r)\right]^{2}\right\} g^{\text {core }}(r) \\
g_{\mathrm{D}}(r)=-\beta w^{\mathrm{el}}(r) g^{\text {core }}(r) .
\end{gathered}
$$

Explicitly, this is

$$
g_{\mathrm{S}}(r)=\left\{1+\frac{1}{2}\left[\frac{\beta\left(q^{\mathrm{eff}}\right)^{2}}{4 \pi \mathcal{E}_{\mathrm{r}}^{\mathrm{eff}} \varepsilon_{0}} \cdot \frac{\mathrm{e}^{-\kappa r}}{r}+\frac{\beta\left(q^{\prime \mathrm{eff}}\right)^{2}}{4 \pi \mathcal{E}_{\mathrm{r}}^{\prime}{ }^{\mathrm{eff}} \varepsilon_{0}} \cdot \frac{\mathrm{e}^{-\kappa^{\prime} r}}{r}\right]^{2}\right\} g^{\mathrm{core}}(r)
$$

and

$$
g_{\mathrm{D}}(r)=-\left[\frac{\beta\left(q^{\mathrm{eff}}\right)^{2}}{4 \pi \mathcal{E}_{\mathrm{r}}^{\mathrm{eff}} \varepsilon_{0}} \cdot \frac{\mathrm{e}^{-\kappa r}}{r}+\frac{\beta\left(q^{\prime \mathrm{eff}}\right)^{2}}{4 \pi \mathcal{E}_{\mathrm{r}}^{\prime}{ }^{\mathrm{eff}} \varepsilon_{0}} \cdot \frac{\mathrm{e}^{-\kappa^{\prime} r}}{r}\right] g^{\text {core }}(r)
$$

This is the basis of the Complete MDE-DH approximation for the RPM. The radial charge distribution is

$$
\begin{aligned}
\rho(r) & =-\frac{\varepsilon_{\mathrm{r}}}{4 \pi}\left[\frac{\kappa^{2} q^{\mathrm{eff}}}{\mathcal{E}_{\mathrm{r}}^{\mathrm{eff}}} \cdot \frac{\mathrm{e}^{-\kappa r}}{r}+\frac{\kappa^{\prime 2} q^{\prime \text { eff }}}{\mathcal{E}_{\mathrm{r}}^{\prime} \text { eff }} \cdot \frac{\mathrm{e}^{-\kappa^{\prime} r}}{r}\right] g^{\text {core }}(r) \\
& =-\frac{\varepsilon_{\mathrm{r}} q}{4 \pi \kappa_{\mathrm{D}}^{2}}\left[\frac{\kappa^{4}}{\mathcal{E}_{\mathrm{r}}^{\mathrm{eff}}} \cdot \frac{\mathrm{e}^{-\kappa r}}{r}+\frac{\kappa^{\prime 4}}{\mathcal{E}_{\mathrm{r}}^{\prime} \text { eff }} \cdot \frac{\mathrm{e}^{-\kappa^{\prime} r}}{r}\right] g^{\mathrm{core}}(r)
\end{aligned}
$$

in this approximation.

In the Simple MDE-DH approximation, where the crossterms between $h^{\text {core }}$ and $w^{\text {el }}$ are neglected, we obtain for $r \geq a$

$$
g_{\mathrm{S}}(r)=1+\frac{1}{2}\left[\beta w^{\mathrm{el}}(r)\right]^{2}+h^{\text {core }}(r)
$$

$$
g_{\mathrm{D}}(r)=-\beta w^{\mathrm{el}}(r)
$$

or explicitly

$$
\begin{aligned}
g_{\mathrm{S}}(r)= & 1 \\
& +\frac{1}{2}\left[\frac{\beta\left(q^{\mathrm{eff}}\right)^{2}}{4 \pi \mathcal{E}_{\mathrm{r}}^{\mathrm{eff}} \varepsilon_{0}} \cdot \frac{\mathrm{e}^{-\kappa r}}{r}+\frac{\beta\left(q^{\prime \text { eff }}\right)^{2}}{4 \pi \mathcal{E}_{\mathrm{r}}^{\prime \text { eff }} \varepsilon_{0}} \cdot \frac{\mathrm{e}^{-\kappa^{\prime} r}}{r}\right]^{2}+h^{\mathrm{core}}(r)
\end{aligned}
$$


and

$$
g_{\mathrm{D}}(r)=-\frac{\beta\left(q^{\text {eff }}\right)^{2}}{4 \pi \mathcal{E}_{\mathrm{r}}^{\text {eff }} \varepsilon_{0}} \cdot \frac{\mathrm{e}^{-\kappa r}}{r}-\frac{\beta\left(q^{\prime \text { eff }}\right)^{2}}{4 \pi \mathcal{E}_{\mathrm{r}}^{\text {eff }} \varepsilon_{0}} \cdot \frac{\mathrm{e}^{-\kappa^{\prime} r}}{r}
$$

for $r \geq a$. By inserting this $g_{\mathrm{D}}(r)$ into eqn (92) and using $\kappa^{2}=$ $\beta n_{\text {tot }} q q^{\text {eff }} / \varepsilon_{\mathrm{r}} \varepsilon_{0}$, we obtain the radial charge density $\rho(r)$ in Ansatz (43). The presence of the square term in $g_{\mathrm{s}}(r)$ in this approximation means that there is a contribution with the decay length $1 / 2 \kappa$ in this function, which is in semi-quantitative agreement with the exact asymptotic analysis in ref. $101(c f$. footnote $\dagger \dagger$ in Section 2).

When one uses the Complete MDE-DH approximation, the expressions for $\mathcal{E}_{\mathrm{r}}^{\text {eff }}$ and $\mathcal{E}_{\mathrm{r}}^{\text {eff }}$ are different from eqn (44)-(49). As shown in Appendix B, the local electroneutrality and the Stillinger-Lovett second moment conditions yield

$$
\begin{aligned}
\frac{\mathcal{E}_{\mathrm{r}}^{\text {eff }}}{\varepsilon_{\mathrm{r}}} & =\frac{M_{1} M_{3}^{\prime}-M_{1}^{\prime} M_{3}}{M_{3}^{\prime}-6 M_{1}^{\prime}} \\
\frac{\mathcal{E}_{\mathrm{r}}^{\prime}}{\varepsilon_{\mathrm{r}}} & =-\frac{M_{1} M_{3}^{\prime}-M_{1}^{\prime} M_{3}}{M_{3}-6 M_{1}}
\end{aligned}
$$

where

$$
\begin{aligned}
& M_{1}=\frac{\kappa^{4}}{\kappa_{\mathrm{D}}^{2}} \int_{a}^{\infty} \mathrm{d} r \mathrm{e}^{-\kappa r} g^{\text {core }}(r) \\
& M_{3}=\kappa^{4} \int_{a}^{\infty} \mathrm{d} r r^{3} \mathrm{e}^{-\kappa r} g^{\text {core }}(r)
\end{aligned}
$$

and the corresponding expressions for $M_{1}{ }^{\prime}$ and $M_{3}{ }^{\prime}$ with $\kappa^{\prime}$ inserted. When $g^{\text {core }}(r) \approx 1$ one obtains $M_{1} \approx 1$ and $M_{3} \approx$ $6 \mathrm{e}^{-\kappa a} \exp _{3}(\kappa a)$ and likewise for $M_{1}{ }^{\prime}$ and $M_{3}{ }^{\prime}$, whereby the result in eqn (46) and (47) is obtained as a good approximation.

In the case of complex-valued decay parameters $\kappa$ and $\kappa^{\prime}=\underline{\kappa}$, we have $M_{1}^{\prime}=\underline{M_{1}}$ and $M_{3}^{\prime}=\underline{M_{3}}$, whereby eqn (102) and (eqn 103) yield $\mathcal{E}_{\mathrm{r}}^{\text {eff }}=\left|\mathcal{E}_{\mathrm{r}}^{\text {eff }}\right| \mathrm{e}^{-\mathrm{i} \vartheta}$ and $\mathcal{E}_{r}^{\text {eff }}=\mathcal{E}_{\mathrm{r}}^{\text {eff }}$. Explicit expressions for $\left|\mathcal{E}_{\mathrm{r}}^{\text {eff }}\right| / \varepsilon_{\mathrm{r}}$ and $\vartheta$ are given in Appendix $\mathrm{B}$ [eqn (109) and (110)]. From eqn (95) and (96) it follows that

$$
g_{\mathrm{S}}(r)=\left\{1+\frac{1}{2}\left[\frac{\left.\beta\left|q^{\text {eff }}\right|\right|^{2}}{2 \pi\left|\mathcal{E}_{\mathrm{r}}^{\text {eff }}\right| \varepsilon_{0}} \cdot \frac{\mathrm{e}^{-\kappa_{\Re} r}}{r} \cos \left(\kappa_{\Im} r+\alpha\right)\right]^{2}\right\} g^{\text {core }}(r)
$$

and

$$
g_{\mathrm{D}}(r)=-\left[\frac{\beta\left|q^{\mathrm{eff}}\right|^{2}}{2 \pi\left|\mathcal{E}_{\mathrm{r}}^{\mathrm{eff}}\right| \varepsilon_{0}} \cdot \frac{\mathrm{e}^{-\kappa_{\Re} r}}{r} \cos \left(\kappa_{\Im} r+\alpha\right)\right] g^{\text {core }}(r),
$$

where $\eta$ is defined from $q^{\text {eff }}=\left|q^{\text {eff }}\right| \mathrm{e}^{-\mathrm{i} \eta}$ and the phase is $\alpha=2 \eta-\vartheta=4 \theta-\vartheta$ since $\eta=2 \theta$ in the RPM, which follows from eqn (51).

\section{B Equations for $\mathcal{E}_{\mathrm{r}}^{\text {eff }}$ and $\mathcal{E}_{\mathrm{r}}^{\text {eff }}$}

In this Appendix we will derive eqn (102) and (103) of Appendix A for $\mathcal{E}_{\mathrm{r}}^{\text {eff }}$ and $\mathcal{E}_{\mathrm{r}}^{\text {eff }}$ of RPM electrolytes in the Complete MDE-DH approximation when $\kappa$ and $\kappa^{\prime}$ are real-valued. We will also obtain the corresponding equations for the complexvalued case.

We have from eqn (97)

$$
\rho(r)=-\frac{q}{4 \pi}\left[\frac{\varepsilon_{\mathrm{r}}}{\mathcal{E}_{\mathrm{r}}^{\mathrm{eff}}} \cdot \frac{\kappa^{4}}{\kappa_{\mathrm{D}}^{2}} \cdot \frac{\mathrm{e}^{-\kappa r}}{r}+\frac{\varepsilon_{\mathrm{r}}}{\mathcal{E}_{\mathrm{r}}^{\prime \mathrm{eff}}} \cdot \frac{\kappa^{\prime 4}}{\kappa_{\mathrm{D}}^{2}} \cdot \frac{\mathrm{e}^{-\kappa^{\prime} r}}{r}\right] g^{\text {core }}(r) .
$$

Let us introduce the unknowns

$$
Y=\frac{\varepsilon_{\mathrm{r}}}{\mathcal{E}_{\mathrm{r}}^{\text {eff }}} \quad \text { and } \quad Y^{\prime}=\frac{\varepsilon_{\mathrm{r}}}{\mathcal{E}_{\mathrm{r}}^{\text {eff }}} .
$$

The local electroneutrality condition yields

$$
Y \cdot \frac{\kappa^{4}}{\kappa_{\mathrm{D}}^{2}} \int_{a}^{\infty} \mathrm{d} r r \mathrm{e}^{-\kappa r} g^{\text {core }}(r)+Y^{\prime} \cdot \frac{\kappa^{\prime}}{\kappa_{\mathrm{D}}^{2}} \int_{a}^{\infty} \mathrm{d} r r \mathrm{e}^{-\kappa^{\prime} r} g^{\text {core }}(r)=1
$$

and the Stillinger-Lovett second moment condition (34), which can be written as $\int d \mathbf{r} r^{2} \rho(r)=-6 \varepsilon_{\mathrm{r}} \varepsilon_{0} /\left(\beta q n_{\text {tot }}\right)=-6 q / \kappa_{\mathrm{D}}^{2}$ in the RPM, yields

$$
Y \cdot \frac{\kappa^{4}}{\kappa_{\mathrm{D}}^{2}} \int_{a}^{\infty} \mathrm{d} r r^{3} \mathrm{e}^{-\kappa r} g^{\text {core }}(r)+Y^{\prime} \cdot \frac{\kappa^{\prime 4}}{\kappa_{\mathrm{D}}^{2}} \int_{a}^{\infty} \mathrm{d} r r^{3} \mathrm{e}^{-\kappa^{\prime} r} g^{\text {core }}(r)=\frac{6}{\kappa_{\mathrm{D}}^{2}} .
$$

This set of equations can be expressed as

$$
\left[\begin{array}{ll}
M_{1} & M_{1}^{\prime} \\
M_{3} & M_{3}^{\prime}
\end{array}\right]\left[\begin{array}{c}
Y \\
Y^{\prime}
\end{array}\right]=\left[\begin{array}{l}
1 \\
6
\end{array}\right],
$$

where the matrix elements $M_{1}$ and $M_{3}$ are defined in eqn (104) and (105) in Appendix A and $M_{1}{ }^{\prime}$ and $M_{3}{ }^{\prime}$ are defined analogously with $\kappa^{\prime}$ inserted. The solution to eqn (108) is $Y=\left(M_{3}{ }^{\prime}-6 M_{1}{ }^{\prime}\right) / D$ and $Y^{\prime}=-\left(M_{3}-6 M_{1}\right) / D$, where $D=M_{1} M_{3}{ }^{\prime}$ $-M_{1}{ }^{\prime} M_{3}$ is the determinant. This solution can be written as in eqn (102) and (eqn 103) in Appendix A.

In the case of complex-valued decay parameters $\kappa=\kappa_{\Re}+$ $\mathrm{i} \kappa_{\mathfrak{I}}=|\kappa| \mathrm{e}^{-i \theta}$ and $\kappa^{\prime}=\underline{\kappa}$ we have $Y^{\prime}=\underline{Y}, M_{1}{ }^{\prime}=\underline{M_{1}}$ and $M_{3}{ }^{\prime}=\underline{M_{3}}$. In this case we can write $Y=\left(\underline{M_{3}}-6 \underline{M_{1}}\right) / D$ and $D=M_{1} \underline{M_{3}}-\underline{M_{1}} M_{3}=M_{1} \underline{M_{3}}-\left(M_{1} \underline{M_{3}}\right)=2 \overline{i \Im}\left(M_{1} \underline{M_{3}}\right)$, where $\mathfrak{I}(\cdot)$ means the imaginary part. Furthermore we have

$$
\begin{aligned}
M_{1} & =|\kappa|^{4} \mathrm{e}^{-\mathrm{i} 4 \theta} \int_{a}^{\infty} \mathrm{d} r \mathrm{e}^{-\kappa_{\Re} r}\left[\cos \left(\kappa_{\Im} r\right)-i \sin \left(\kappa_{\Im} r\right)\right] g^{\mathrm{core}}(r) / \kappa_{\mathrm{D}}{ }^{2} \\
& \equiv|\kappa|^{4} \mathrm{e}^{-\mathrm{i} 4 \theta}\left[G_{1}^{\mathrm{cos}}-i G_{1}^{\mathrm{sin}}\right]
\end{aligned}
$$

and

$$
\begin{aligned}
M_{3} & =|\kappa|^{4} \mathrm{e}^{-\mathrm{i} 4 \theta} \int_{a}^{\infty} \mathrm{d} r r^{3} \mathrm{e}^{-\kappa_{\Re} r}\left[\cos \left(\kappa_{\Im} r\right)-i \sin \left(\kappa_{\Im} r\right)\right] g^{\operatorname{core}}(r) \\
& \equiv|\kappa|^{4} \mathrm{e}^{-\mathrm{i} 4 \theta}\left[G_{3}^{\mathrm{cos}}-i G_{3}^{\mathrm{sin}}\right],
\end{aligned}
$$

where $G_{\nu}^{\cos }$ and $-G_{\nu}^{\text {sin }}$ are defined as the real and the complex parts of the integrals, respectively. We hence obtain

$$
\frac{\varepsilon_{\mathrm{r}}}{\left|\mathcal{E}_{\mathrm{r}}^{\mathrm{eff}}\right|} \mathrm{e}^{i \vartheta}=Y=\frac{\mathrm{e}^{i(4 \theta+3 \pi / 2)}|\kappa|^{4}\left[G_{3}^{\mathrm{cos}}-6 G_{1}^{\cos }+i\left(G_{3}^{\sin }-6 G_{1}^{\mathrm{sin}}\right)\right]}{2|\kappa|^{8}\left[G_{1}^{\cos } G_{3}^{\sin }-G_{1}^{\sin } G_{3}^{\cos }\right]},
$$


where we have used $-i=\mathrm{e}^{\mathrm{i} 3 \pi / 2}$. By extracting $|Y|$ and $\arg (Y)$ we obtain

$$
\frac{\left|\mathcal{E}_{\mathrm{r}}^{\mathrm{eff}}\right|}{\varepsilon_{\mathrm{r}}}=\frac{2|\kappa|^{4}\left|G_{1}^{\mathrm{cos}} G_{3}^{\mathrm{sin}}-G_{1}^{\sin } G_{3}^{\cos }\right|}{\left[\left(G_{3}^{\cos }-6 G_{1}^{\cos }\right)^{2}+\left(G_{3}^{\sin }-6 G_{1}^{\sin }\right)^{2}\right]^{1 / 2}}
$$

and

$$
\vartheta=4 \theta+3 \pi / 2+\arctan \left(\frac{G_{3}^{\sin }-6 G_{1}^{\sin }}{G_{3}^{\cos }-6 G_{1}^{\cos }}\right)
$$

when $G_{1}^{\cos } G_{3}^{\text {sin }}-G_{1}^{\text {sin }} G_{3}^{\cos } \geq 0$ (otherwise there is a further term $-\pi$ in $\vartheta$ ).

\section{Theoretical considerations for $U^{\text {ex }}$, $\gamma_{ \pm}$and $\phi$}

Here we will present the theoretical details regarding the thermodynamic entities. The electrostatic contribution $\mu_{i}^{\text {ex,el }}$ to the excess chemical potential is, as we have seen, the free energy change (= the reversible work) when the charge of a sphere inserted in the electrolyte is changed from 0 to $q_{i}$. It can be calculated via a coupling parameter integration

$$
\begin{aligned}
\mu_{i}^{\mathrm{ex}, \mathrm{el}} & =\sum_{j} \int_{0}^{1} \mathrm{~d} \xi \int \mathrm{d} \mathbf{r} \frac{\partial\left[\xi u_{i j}^{\mathrm{el}}(r)\right]}{\partial \xi} n_{j} g_{i j}(r ; \xi) \\
& =\int_{0}^{1} \mathrm{~d} \xi \int_{a}^{\infty} \mathrm{d} r 4 \pi r^{2} \frac{q_{i} \sum_{j} q_{j} n_{j} g_{i j}(r ; \xi)}{4 \pi \varepsilon_{\mathrm{r}} \varepsilon_{0} r}=\int_{0}^{1} \mathrm{~d} \xi \int_{a}^{\infty} \mathrm{d} r r \frac{q_{i} \rho_{i}(r ; \xi)}{\varepsilon_{\mathrm{r}} \varepsilon_{0}},
\end{aligned}
$$

where $u_{i j}^{\mathrm{el}}(r)=q_{i} q_{j} /\left(4 \pi \varepsilon_{\mathrm{r}} \varepsilon_{0} r\right)$ is the electrostatic pair potential, $\xi$ is the coupling parameter $1 \leq \xi \leq 1, g_{i j}(r ; \xi)$ is the pair distribution function around a partially charged ion with charge $\xi q_{i}$ placed at the origin and $\rho_{i}(r ; \xi)$ is the charge density around this ion. All other ions are fully charged throughout. The corresponding contribution to the mean activity coefficient $\gamma_{ \pm}$is

$$
\ln \gamma_{ \pm}^{\mathrm{el}} \equiv \frac{\mu_{ \pm}^{\mathrm{el}, \mathrm{ex}}}{k_{\mathrm{B}} T}=\beta \sum_{i} x_{i} \mu_{i}^{\mathrm{ex}, \mathrm{el}}=\frac{\beta}{n_{\mathrm{tot}}} \int_{0}^{1} \mathrm{~d} \xi \int_{a}^{\infty} \mathrm{d} r r \sum_{i} \frac{n_{i} q_{i} \rho_{i}(r ; \xi)}{\varepsilon_{\mathrm{r}} \varepsilon_{0}}
$$

For approximations where the charge density $\rho_{i}(r)$ is proportional to the ionic charge $q_{i}$, like the $\mathrm{DH}, \mathrm{MDH}$ and $\mathrm{MDE}-\mathrm{DH}$ approximations, we have $\rho_{i}(r ; \xi)=\xi \rho_{i}(r)$. Then, the integration over $\xi$ contributes with a factor $\int_{0}^{1} \mathrm{~d} \xi \xi=1 / 2$ so we have

$$
\ln \gamma_{ \pm}^{\mathrm{el}}=\frac{\beta}{2 n_{\mathrm{tot}}} \int_{a}^{\infty} \mathrm{d} r r \sum_{i} \frac{n_{i} q_{i} \rho_{i}(r)}{\varepsilon_{\mathrm{r}} \varepsilon_{0}}
$$

for such linear approximations. In the $\mathrm{DH}$ and $\mathrm{MDH}$ approximations this gives $\ln \gamma_{ \pm}^{\text {el }}$ in eqn (62) and (63), respectively.

In the Simple MDE-DH approximation based on the Ansatz (43), we take $\rho_{i}(r)$ from the second line in this equation and obtain after simplification

$$
\ln \gamma_{ \pm}^{\mathrm{el}}=-\frac{\varepsilon_{\mathrm{r}}}{8 \pi n_{\mathrm{tot}}}\left[\frac{\kappa^{3}}{\mathcal{E}_{\mathrm{r}}^{\mathrm{eff}}} \mathrm{e}^{-\kappa a}+\frac{\kappa^{\prime 3}}{\mathcal{E}_{\mathrm{r}}^{\prime \mathrm{eff}}} \mathrm{e}^{-\kappa^{\prime} a}\right]
$$

where we have made use of eqn (19) and its primed counterpart eqn (22). Using eqn (33), we can alternatively write this expression as eqn (64). When the charge density is oscillatory and $\kappa$ and $\kappa^{\prime}$ are complex-valued, these expressions for $\ln \gamma_{ \pm}^{\text {el }}$ can be used as they stand, but they can alternatively be written in a more convenient manner as

$$
\ln \gamma_{ \pm}^{\mathrm{el}}=-\frac{\kappa_{\mathrm{D}}^{2}}{8 \pi n_{\mathrm{tot}}} \cdot \frac{|\kappa a|^{2}+\kappa_{\Re} a-\kappa_{\Im} a \tan \vartheta}{a|1+\kappa a|^{2}}
$$

$(\kappa$ complex $)$,

which can be explicitly expressed in terms of $\kappa_{\Re}$ and $\kappa_{\mathfrak{I}}$ as

$$
\ln \gamma_{ \pm}^{\mathrm{el}}=-\frac{\kappa_{\mathrm{D}}^{2}}{8 \pi n_{\mathrm{tot}}} \cdot \frac{\left(\kappa_{\Re} a\right)^{2}+\left(\kappa_{\Im} a\right)^{2}+\kappa_{\Re} a-\kappa_{\Im} a \tan \vartheta}{a\left[\left(1+\kappa_{\Re} a\right)^{2}+\left(\kappa_{\Im} a\right)^{2}\right]}
$$

$(\kappa$ complex $)$.

Note that $\tan \vartheta$ can be obtained in terms of $\kappa_{\Re}$ and $\kappa_{\mathfrak{I}}$ from eqn (49).

The average internal energy $U$ of the system is

$$
U=U^{\mathrm{ideal}}+U^{\mathrm{ex}}=\frac{3 N_{\mathrm{tot}} k_{\mathrm{B}} T}{2}+\frac{1}{2} \sum_{i, j} N_{i} n_{j} \int \mathrm{d} \mathbf{r} u_{i j}^{\mathrm{el}}(r) g_{i j}(r) .
$$

The second term on the rhs can be written as

$$
\frac{U^{\mathrm{ex}}}{N_{\mathrm{tot}}}=\frac{1}{2 n_{\mathrm{tot}}} \int_{a}^{\infty} \mathrm{d} r r \sum_{i} \frac{n_{i} q_{i} \rho_{i}(r)}{\varepsilon_{\mathrm{r}} \varepsilon_{0}},
$$

where we have used $N_{i} / N_{\text {tot }}=n_{i} / n_{\text {tot }}$. By comparing with eqn (112), which is valid for approximations where the charge density $\rho_{i}(r)$ is proportional to the ionic charge $q_{i}$, we see that $\beta U^{\text {ex }} / N_{\text {tot }}=\ln \gamma_{ \pm}^{\text {el }}$ in such cases. Consequently, in the Simple MDE-DH approximation $\beta U^{\mathrm{ex}} / N_{\text {tot }}$ is equal to the rhs of (eqn 113), so (eqn 65) follows when (eqn 33) is used. When $\kappa$ and $\kappa^{\prime}$ are complex, $\beta U^{\mathrm{ex}} / N_{\text {tot }}$ can be calculated from rhs of eqn (114).

Next, we treat the osmotic coefficient. The excess pressure of the electrolyte consists of two parts $P^{\mathrm{ex}}=P^{\text {cont }}+P^{\mathrm{el}}$, where $P^{\text {cont }}$ is the contact pressure evaluated at the hard core surfaces of the ions

$$
P^{\mathrm{cont}}=\frac{2 \pi a^{3} k_{\mathrm{B}} T}{3} \sum_{i, j} n_{i} n_{j} g_{i j}(a)
$$

and

$$
P^{\mathrm{el}}=-\frac{1}{6} \sum_{i, j} n_{i} n_{j} \int \mathrm{d} \mathbf{r} r \frac{\mathrm{d} u_{i j}^{\mathrm{el}}(r)}{\mathrm{d} r} g_{i j}(r)=\frac{1}{6} \int_{a}^{\infty} \mathrm{d} r r \sum_{i} \frac{n_{i} q_{i} \rho_{i}(r)}{\varepsilon_{\mathrm{r}} \varepsilon_{0}} .
$$

The excess osmotic coefficient, $\phi^{\mathrm{ex}}=\phi^{\text {cont }}+\phi^{\mathrm{el}}$ is accordingly given by

$$
\phi^{\mathrm{ex}}=\frac{2 \pi a^{3}}{3 n_{\mathrm{tot}}} \sum_{i, j} n_{i} n_{j} g_{i j}(a)+\frac{\beta}{6 n_{\mathrm{tot}}} \int_{a}^{\infty} \mathrm{d} r r \sum_{i} \frac{n_{i} q_{i} \rho_{i}(r)}{\varepsilon_{\mathrm{r}} \varepsilon_{0}},
$$

where the first term is $\phi^{\text {cont }}=P^{\text {cont }} / P^{\text {ideal }}$ and the second is $\phi^{\mathrm{el}}=P^{\mathrm{el}} / P^{\text {ideal }}$. 
In the Simple MDE-DH approximation based on the Ansatz (43) we obtain

$$
\phi^{\mathrm{el}}=-\frac{\varepsilon_{\mathrm{r}}}{24 \pi n_{\mathrm{tot}}}\left[\frac{\kappa^{3}}{\mathcal{E}_{\mathrm{r}}^{\mathrm{eff}}} \mathrm{e}^{-\kappa a}+\frac{\kappa^{\prime 3}}{\mathcal{E}_{\mathrm{r}}^{\prime}} \mathrm{e}^{-\kappa^{\prime} a}\right],
$$

which can be written as eqn (69). For the case of complexvalued $\kappa$ and $\kappa^{\prime}$, this expression is still valid, but it can be written analogously to eqn (114) [the prefactor for $\phi^{\mathrm{el}}$ has $24 \pi$ instead $8 \pi$ in the denominator].

As mentioned in Section 4.3, the $\mathrm{DH}$ and $\mathrm{MDH}$ approximations show very poor consistency for $\ln \gamma_{ \pm}$obtained from eqn (112) and that obtained from the osmotic coefficient via eqn (71). The reason for this lies in the handling of the electrostatic contributions. Both approximations treat the core contribution consistently, because we have

$$
\phi^{\mathrm{cont}}=\frac{2 \pi a^{3} n_{\mathrm{tot}}}{3} \quad(\mathrm{DH}, \mathrm{MDH}),
$$

which yields the contribution $4 \pi a^{3} n_{\text {tot }} / 3$ when inserted into eqn (71), that is, the same as $\ln \gamma_{ \pm}^{\text {core }}$ given in eqn (59). Eqn (120) follows from the fact that $\sum_{j} n_{j} g_{i j}(r)=n_{\text {tot }}$ for $r \geq a$ in the $\mathrm{DH}$ and $\mathrm{MDH}$ approximations, so we have $\sum_{i, j} n_{i} n_{j} g_{i j}(a)=\left(n_{\mathrm{tot}}\right)^{2}$. The inconsistency originates from the fact that $\phi^{\mathrm{el}}$ inserted into eqn (71) does not give the same electrostatic contribution as the one obtained from eqn (111). The latter is, in fact, a general feature of linear approximations, where the charge density $\rho_{i}(r)$ is proportional to $q_{i}$. This can be realized as follows.

For these kinds of approximations, $\ln \gamma_{ \pm}^{\text {el }}$ is calculated from eqn (112) and a comparison with the second term in eqn (118) shows that the integral is the same in both equations and that the prefactors differ only by a factor of $1 / 3$, which implies that $\ln \gamma_{ \pm}^{\mathrm{el}}=3 \phi^{\mathrm{el}}$. This means that in eqn (71) one obtains one third of $\ln \gamma_{ \pm}^{\text {el }}$ from the first term on the rhs and that the second term must yield two thirds of $\ln \gamma_{ \pm}^{\text {el }}$ in order to have consistency. Thus the integral in this second term must be equal to one third of $\ln \gamma_{ \pm}^{\mathrm{el}}$, that is, equal to $\phi^{\mathrm{el}}$. Consistency is achieved provided the function $\phi^{\mathrm{ex}}\left(n_{\text {tot }}\right)$ satisfies $\int_{0}^{s} \mathrm{~d} t \phi^{\mathrm{ex}}\left(t^{2}\right) / t=\phi^{\mathrm{ex}}\left(s^{2}\right)$, where we have made the substitutions $n_{\text {tot }}{ }^{\prime}=t^{2}$ and $n_{\text {tot }}=s^{2}$. This implies that $\phi^{\mathrm{el}}\left(s^{2}\right)=K s$, that is, $\phi^{\mathrm{el}}\left(n_{\text {tot }}\right)=K \sqrt{n_{\text {tot }}}$, where $K$ is a constant. This is satisfied for the Debye-Hückel limiting law (2) for $\phi^{\mathrm{ex}}=\phi-1$ at infinite dilution, but not otherwise. Since the limiting law is an exact result of statistical mechanics, it must obey the consistency requirement, but no other linear approximation can be consistent in the sense that $\phi^{\mathrm{el}}$ inserted into eqn (71) yields $\ln \gamma_{ \pm}^{\text {el }}$ (except in the limit of infinite dilution).

Since the charge density $\rho_{i}(r)$ is proportional to $q_{i}$ in the MDE-DH approximations, they share the same feature, that is, $\ln \gamma_{ \pm}^{\mathrm{el}}$ is not obtained from $\phi^{\mathrm{el}}$ via the integration in eqn (71). However, $\phi^{\mathrm{el}}$ is not the only contribution to $\phi^{\mathrm{ex}}$ where electrostatics matter in these approximations; there is a further contribution due to the quadratic terms in $w^{\text {el }}$ in eqn (55) and (58) in the complete and simple versions of this approximation, respectively. We will start by considering the Simple MDE-DH approximation and see that there is an electrostatic part in $\phi^{\text {contact }}$ due to the presence of the quadratic term $\left[\beta w^{\mathrm{el}}(r)\right] / 2$ in eqn (58) and hence in eqn (98). This is an important contribution that is necessary for the good agreement between the MDE-DH and MC data for $\phi$ in Fig. 8 and 9 and for the good consistency between the two different manners to calculate $\ln \gamma_{ \pm}$.

From eqn (118) we obtain for the RPM

$$
\begin{gathered}
\phi^{\mathrm{cont}}=\frac{2 \pi a^{3}}{3} n_{\mathrm{tot}} g_{\mathrm{S}}(a) \\
\phi^{\mathrm{el}}=\frac{\kappa_{\mathrm{D}}^{2}}{6} \int_{a}^{\infty} \mathrm{d} r r g_{\mathrm{D}}(r)=\frac{\beta q}{6 \varepsilon_{\mathrm{r}} \varepsilon_{0}} \int_{a}^{\infty} \mathrm{d} r r \rho(r),
\end{gathered}
$$

where $g_{\mathrm{S}}$ and $g_{\mathrm{D}}$ are defined in eqn (89) and we have used (eqn 92). It follows from eqn (98) that in the Simple MDE-DH approximation we have

$$
\begin{aligned}
\phi^{\mathrm{cont}} & =\frac{2 \pi a^{3} n_{\mathrm{tot}}}{3}\left[\frac{\left[\beta w^{\mathrm{el}}(a)\right]^{2}}{2}+g^{\mathrm{core}}(a)\right] \\
& =\frac{\pi a^{3} n_{\mathrm{tot}}}{3}\left[\beta w^{\mathrm{el}}(a)\right]^{2}+\phi^{\mathrm{core}},
\end{aligned}
$$

where $\phi^{\text {core }}=2 \pi a^{3} n_{\text {totg }} g^{\text {core }}(a) / 3$. By inserting $w^{\text {el }}(r)$ from eqn (54) into eqn (123) we obtain

$$
\phi^{\text {cont }}=\frac{\pi a^{3} n_{\mathrm{tot}}}{3}\left[\frac{\beta\left(q^{\mathrm{eff}}\right)^{2}}{4 \pi \mathcal{E}_{\mathrm{r}}^{\mathrm{eff}} \varepsilon_{0}} \cdot \frac{\mathrm{e}^{-\kappa a}}{a}+\frac{\beta\left(q^{\prime \text { eff }}\right)^{2}}{4 \pi \mathcal{E}_{\mathrm{r}}^{\prime \text { eff }} \varepsilon_{0}} \cdot \frac{\mathrm{e}^{-\kappa^{\prime} a}}{a}\right]^{2}+\phi^{\text {core }}
$$

with $\phi^{\text {core }}$ given by eqn (70). In the Simple MDE-DH approximation, we accordingly have $\phi=1+\phi^{\text {cont }}+\phi^{\text {el }}$ with $\phi^{\text {cont }}$ from eqn (124) and $\phi^{\text {el }}$ from eqn (119).

In the Complete MDE-DH approximation it follows from eqn (93) and (121) that

$$
\begin{aligned}
\phi^{\mathrm{cont}} & =\frac{2 \pi a^{3}}{3} n_{\mathrm{tot}}\left[1+\frac{\left[\beta w^{\mathrm{el}}(a)\right]^{2}}{2}\right] g^{\mathrm{core}}(a) \\
& =\left[1+\frac{\left[\beta w^{\mathrm{el}}(a)\right]^{2}}{2}\right] \phi^{\mathrm{core}}
\end{aligned}
$$

and we have from eqn (94) and (122)

$$
\phi^{\mathrm{el}}=-\frac{\beta \kappa_{\mathrm{D}}^{2}}{6} \int_{a}^{\infty} \mathrm{d} r r w^{\mathrm{el}}(r) g^{\text {core }}(r) .
$$

When $w^{\mathrm{el}}(r)$ from eqn (54) is inserted, $\phi^{\mathrm{el}}$ can be written in terms of $M_{1}$ and $M_{1}{ }^{\prime}$, which are integrals involving $g^{\text {core }}$ defined in eqn (104) in Appendix A. Furthermore, in this approximation

$$
\ln \gamma_{ \pm}^{\mathrm{el}}=-\frac{\beta \kappa_{\mathrm{D}}^{2}}{2} \int_{a}^{\infty} \mathrm{d} r r w^{\mathrm{el}}(r) g^{\mathrm{core}}(r)
$$

which contains the same integral, and $\ln \gamma_{ \pm}^{\text {core }}$ is given by eqn (60). 


\section{Asymptotic formulas at high dilution}

In this appendix we consider $\kappa^{2} / \kappa_{\mathrm{D}}^{2}$ and $\mathcal{E}_{\mathrm{r}}^{\text {eff }} / \varepsilon_{\mathrm{r}}$ in very dilute solutions of symmetric electrolytes of ions with diameter $a$ and derive approximate asymptotic formulas for these entities when $\tau=\kappa_{\mathrm{D}} a \rightarrow 0$. These formulas will be extracted from eqn (76) and (77), which can be written as

$$
\begin{gathered}
\frac{\tau^{2}}{\beta_{R}} \int_{1}^{\infty} \mathrm{d} R R^{2} \sinh \left(\beta_{R} X^{4} Y \cdot \frac{\mathrm{e}^{-X \tau R}}{R}\right)=1 \\
\frac{\tau^{4}}{6 \beta_{R}} \int_{1}^{\infty} \mathrm{d} R R^{4} \sinh \left(\beta_{R} X^{4} Y \cdot \frac{\mathrm{e}^{-X \tau R}}{R}\right)=1,
\end{gathered}
$$

where $X=\kappa / \kappa_{\mathrm{D}}$ and $Y=\varepsilon_{\mathrm{r}} / \mathcal{E}_{\mathrm{r}}^{\text {eff }}$. We will extract all contributions to order $\tau^{2}$ or less, that is, up to and including contributions that are proportional to the total ionic density $n_{\text {tot }}$. We do this by expanding $\sinh x=x+x^{3} / 3 !+x^{5} / 5 !+\ldots$ and expressing the integrals involving $\left[\mathrm{e}^{-X \tau R} / R\right]^{\nu}$ in terms of known functions, namely

$$
\begin{aligned}
\int_{1}^{\infty} \mathrm{d} R R^{2}\left[\frac{\mathrm{e}^{-X \tau R}}{R}\right]^{\nu} & =\int_{1}^{\infty} \mathrm{d} R R^{2-\nu} \mathrm{e}^{-\nu X \tau R} \\
& =(\nu X \tau)^{\nu-3} \Gamma(3-\nu, \nu X \tau)=\mathcal{F}_{\nu+2}(\nu X \tau)
\end{aligned}
$$

and

$$
\begin{aligned}
\int_{1}^{\infty} \mathrm{d} R R^{4}\left[\frac{\mathrm{e}^{-X \tau R}}{R}\right]^{\nu} & =\int_{1}^{\infty} \mathrm{d} R R^{4-\nu} \mathrm{e}^{-\nu X \tau R} \\
& =(\nu X \tau)^{\nu-5} \Gamma(5-\nu, \nu X \tau)=\mathcal{F}_{\nu}(\nu X \tau),
\end{aligned}
$$

where $\Gamma(m, x)=\int_{x}^{\infty} t^{m-1} \mathrm{e}^{-t}$ is the incomplete Gamma function and where we have defined $\mathcal{F}_{m}(x)$ from

$$
\mathcal{F}_{m}(x) \equiv x^{m-5} \Gamma(5-m, x)= \begin{cases}6 \mathrm{e}^{-x} \exp _{3}(x) / x^{4}, & m=1 \\ \mathrm{e}^{-x}(1+x) / x^{2}, & m=3 \\ E_{1}(x), & m=5,\end{cases}
$$

where $E_{1}(x)$ is the exponential integral $E_{1}(x) \equiv \int_{x}^{\infty} \mathrm{d} t \mathrm{e}^{-t} / t$. The function $\mathcal{F}_{m}(x)$ for $m>5$ has the limit

$$
\lim _{x \rightarrow 0} \mathcal{F}_{m}(x)=\frac{1}{m-5} \text { when } m>5,
$$

but for $m \leq 5$ it diverges when $x \rightarrow 0$. For $m=5$ the divergence is logarithmic since $\mathcal{F}_{5}(x)=E_{1}(x) \sim-\mathcal{C}_{\mathrm{Eu}}-\ln x+x$ when $x \rightarrow 0$, where $\mathcal{C}_{\mathrm{Eu}} \approx 0.5772$ is Euler's constant.

Using these results in eqn (128), which is the local electroneutrality condition, we obtain

$$
\sum_{\text {odd } \nu} \frac{\beta_{R}^{\nu-1} \tau^{2}}{\nu !} X^{4 \nu} Y^{\nu} \mathcal{F}_{\nu+2}(\nu X \tau)=1
$$

and likewise eqn (129), which is the Stillinger-Lovett condition, can be written

$$
\frac{1}{6} \sum_{\text {odd } \nu} \frac{\beta_{R}^{\nu-1} \tau^{4}}{\nu !} X^{4 \nu} Y^{\nu} \mathcal{F}_{\nu}(\nu X \tau)=1
$$

Since $X=\kappa / \kappa_{\mathrm{D}} \rightarrow 1$ and $Y=\varepsilon_{\mathrm{r}} / \mathcal{E}_{\mathrm{r}}^{\text {eff }} \rightarrow 1$ when $\tau \rightarrow 0$, we have $X=1+\alpha_{X}(\tau)$ and $Y=1+\alpha_{Y}(\tau)$, where the as yet unknown functions $\alpha_{X}(\tau) \rightarrow 0$ and $\alpha_{Y}(\tau) \rightarrow 0$ when $\tau \rightarrow 0$. Hence

$$
X^{l} \sim 1+l \alpha_{X}(\tau) \text { and } Y^{l} \sim 1+l \alpha_{Y}(\tau) \text { when } \tau \rightarrow 0 .
$$

We start with the Stillinger-Lovett condition (133). We first note that $\mathcal{F}_{5}(5 X \tau) \sim-\ln (5 X \tau)=-\ln (5 X)-\ln \tau \sim-\ln \tau$ when $\tau \rightarrow 0$. This implies that the term for $\nu=5$ in eqn (133) decays like $-\tau^{4} \ln \tau$ in this limit. This factor decays faster than $\tau^{2}$ so the $\nu=5$ term can be skipped. Using eqn (131) we can see that the terms for $\nu>5$ decay like $\tau^{4}$, so they can also be skipped. Thus there remains only the terms for $\nu=1$ and 3 .

The $\nu=1$ term in the left-hand side (lhs) of eqn (133) is

$$
\frac{\tau^{4}}{6} X^{4} Y \mathcal{F}_{1}(X \tau)=Y \mathrm{e}^{-X \tau} \exp _{3}(X \tau)
$$

where we have used eqn (130), and the $\nu=3$ term is

$$
\frac{\beta_{R}^{2} \tau^{4}}{6 \cdot 3 !} X^{12} Y^{3} \mathcal{F}_{3}(3 X \tau)=\frac{\beta_{R}^{2} \tau^{2}}{324} X^{10} Y^{3} \mathrm{e}^{-3 X \tau}(1+3 X \tau) .
$$

We now insert these terms into eqn (133), whereby we skip contributions that decay faster to zero than $\tau^{2}$ in order to extract the asymptote. In the $\nu=3$ term, the factor $\mathrm{e}^{-3 X \tau}(1+3 X \tau)$ decays when $\tau \rightarrow 0$ like

$$
\begin{aligned}
\mathrm{e}^{-3 X \tau}(1+3 X \tau) \sim 1- & (3 X \tau)^{2} / 2 \sim 1-(3 \tau)^{2} / 2-\alpha_{X}(\tau)(3 \tau)^{2} \\
& \sim 1-(3 \tau)^{2} / 2,
\end{aligned}
$$

where we have used eqn (134). Furthermore, $X^{10} \sim 1+10 \alpha_{X}(\tau)$ and $Y^{3} \sim 1+3 \alpha_{Y}(\tau)$. This means that $\tau^{2} X^{10} Y^{3} \mathrm{e}^{-3 X \tau}(1+3 X \tau)$ decays like $\tau^{2}$ and we can conclude that the $\nu=3$ term decays like $\beta_{R}{ }^{2} \tau^{2} / 324$. By inserting this result and the $\nu=1$ term into eqn (133) and rearranging we obtain

$$
Y \sim \frac{1-\beta_{R}{ }^{2} \tau^{2} / 324}{\mathrm{e}^{-X \tau} \exp _{3}(X \tau)} \text { when } \tau \rightarrow 0 .
$$

Now, $\mathrm{e}^{-X \tau} \exp _{3}(X \tau) \sim 1-(X \tau)^{4} / 24 \sim 1-\tau^{4} / 24$, so $\left[\mathrm{e}^{-X \tau} \exp _{3}(X \tau)\right]^{-1} \sim 1+\tau^{4} / 24$, so we can finally conclude that

$$
Y=\frac{\varepsilon_{\mathrm{r}}}{\mathcal{E}_{\mathrm{r}}^{\mathrm{eff}}} \sim 1-\frac{\beta_{R}^{2} \tau^{2}}{324} \quad \text { when } \tau \rightarrow 0
$$

to the order $\tau^{2}$.

We now proceed with the local electroneutrality condition (132). The $\nu=1$ term in the lhs of this equation is

$$
\tau^{2} X^{4} Y \mathcal{F}_{3}(X \tau)=X^{2} Y \mathrm{e}^{-X \tau}(1+X \tau)
$$

and the $\nu=3$ term is

$$
\frac{\beta_{R}^{2} \tau^{2}}{3 !} X^{12} Y^{3} \mathcal{F}_{5}(3 X \tau)=\frac{\beta_{R}^{2} \tau^{2}}{6} X^{12} Y^{3} E_{1}(3 X \tau) .
$$

Analogously to the previous arguments we find that $\tau^{2} X^{12} Y^{3}$ decays like $\tau^{2}$, so the entire $\nu=3$ term decays like up

$$
\frac{\beta_{R}^{2} \tau^{2}}{6} E_{1}(3 X \tau) \sim-\frac{\beta_{R}^{2} \tau^{2}}{6}\left[\mathcal{C}_{\mathrm{Eu}}+\ln (3 \tau)\right]
$$


to the order $\tau^{2}$. There are also contributions of the order $\tau^{2}$ from the terms with $\nu \geq 5$. The factor $\tau^{2} X^{4 \nu} Y^{\nu}$ in these terms decays like $\tau^{2}$ and by using eqn (131) we see that the terms decay when $\tau \rightarrow 0$ like

$$
\frac{\beta_{R}^{\nu-1} \tau^{2}}{\nu !} \mathcal{F}_{\nu+2}(\nu X \tau) \sim \beta_{R}{ }^{2} \tau^{2} \frac{\beta_{R}^{\nu-3}}{\nu !(\nu-3)} \quad \text { when } \nu \geq 5 .
$$

The leading contribution from all terms with $\nu \geq 5$ is accordingly equal to $\beta_{R}{ }^{2} \tau^{2} s\left(\beta_{R}{ }^{2}\right)$, where we have defined

$$
s(x)=\sum_{\text {odd } \nu \geq 5} \frac{x^{(\nu-3) / 2}}{\nu !(\nu-3)}=\sum_{l=1}^{\infty} \frac{x^{l}}{(2 l+3) ! 2 l},
$$

where we have made the substitution $\nu=2 l+3$. By gathering these results and inserting them together with the $\nu=1$ term into eqn (132), we obtain after rearrangement

$$
X^{2} \sim \frac{1+\beta_{R}^{2} \tau^{2}\left(\left[\mathcal{C}_{\mathrm{Eu}}+\ln (3 \tau)\right] / 6-s\left(\beta_{R}^{2}\right)\right)}{Y \mathrm{e}^{-X \tau}(1+X \tau)} \quad \text { when } \tau \rightarrow 0 .
$$

Now, from eqn (135) it follows that $1 / Y$ decays like $1+\beta_{R}{ }^{2} \tau^{2} / 324$ and we have $\left[\mathrm{e}^{-X \tau}(1+X \tau)\right]^{-1} \sim 1+(X \tau)^{2} / 2 \sim 1+\tau^{2} / 2$. Thus we can finally conclude that

$$
X^{2}=\frac{\kappa^{2}}{\kappa_{\mathrm{D}}^{2}} \sim 1+\beta_{R}{ }^{2} \tau^{2}\left(\frac{\mathcal{C}_{\mathrm{Eu}}+\ln (3 \tau)}{6}-s\left(\beta_{R}{ }^{2}\right)+\frac{1}{324}\right)+\frac{\tau^{2}}{2}
$$

to the order $\tau^{2}$. Eqn (135) and (137) constitute the wanted result, which can be written as eqn (82) and (81), respectively, in the main text.

\section{Acknowledgements}

I want to thank Andrés González de Castilla for giving me the inspiration to do this work, for interesting discussions on practical applications of the theory of electrolytes and for helpful comments on the manuscript of this paper. Gregor Trefalt is cordially thanked for sending me preprints and raw data for the measurements of the decay lengths in dilute electrolytes and for useful comments on the manuscript. I also want to thank Sture Nordholm for his constructive remarks on the paper.

\section{References}

$1 \mathrm{M} . \mathrm{Su}$ and Y. Wang, Commun. Theor. Phys., 2020, 72, 067601.

2 L. L. Lee, Molecular Thermodynamics of Electrolyte Solutions, World Scientific Publishing, Singapore, 2008.

3 L. M. Varela, M. García and V. Mosquera, Phys. Rep., 2003, 382, 1.

4 L. Lue, in Variational Methods in Molecular Modeling, ed. J. Wu, Springer, Singapore, 2017, p. 137.

5 B. Maribo-Mogensen, G. M. Kontogeorgis and K. Thomsen, Ind. Eng. Chem. Res., 2012, 51, 5353.

6 G. M. Kontogeorgis, B. Maribo-Mogensen and K. Thomsen, Fluid Phase Equilib., 2018, 462, 130.
7 P. Debye and E. Hückel, Phys. Z., 1923, 24, 185.

8 K. S. Pitzer, J. Phys. Chem., 1973, 77, 268.

9 K. S. Pitzer and G. Mayorga, J. Phys. Chem., 1973, 77, 2300.

10 K. S. Pitzer, Acc. Chem. Res., 1977, 10, 371.

11 J. C. Rasiah, D. N. Card and J. P. Valleau, J. Chem. Phys., 1972, 56, 248.

12 G. Hummer and D. M. Soumpasis, J. Chem. Phys., 1993, 98, 581.

13 J. G. Kirkwood, Chem. Rev., 1936, 19, 275.

14 J. G. Kirkwood and J. C. Poirier, J. Phys. Chem., 1954, 58, 591.

15 G. A. Martynov, Sov. Phys. - Usp., 1967, 10, 171.

16 C. W. Outhwaite in Statistical Mechanics: A Specialist Periodical Report, ed. K. Singer, The Chemical Society, London, 1975, vol. 2, p. 188.

17 P. Keblinski, J. Eggebrecht, D. Wolf and S. R. Phillpot, J. Chem. Phys., 2000, 113, 282.

18 J. Ulander and R. Kjellander, J. Chem. Phys., 2001, 114, 4893.

19 F. Coupette, A. A. Lee and A. Härtel, Phys. Rev. Lett., 2018, 121, 075501.

20 Supplementary material for ref. 19 at http://link.aps.org/ supplemental/10.1103/PhysRevLett.121.075501.

21 J. Ennis, PhD thesis, Australian National University, 1993.

22 J. Ennis, R. Kjellander and D. J. Mitchell, J. Chem. Phys., 1995, 102, 975.

23 J. Ulander and R. Kjellander, J. Chem. Phys., 1998, 109, 9508.

24 R. J. F. Leote de Carvalho and R. Evans, Mol. Phys., 1994, 83, 619.

25 E. Waismann and J. L. Lebowitz, J. Chem. Phys., 1972, 56, 3086.

26 E. Waismann and J. L. Lebowitz, J. Chem. Phys., 1972, 56, 3093.

27 C. W. Outhwaite and V. C. L. Hutson, Mol. Phys., 1975, 29, 1521.

28 L. Blum. in Theoretical Chemistry, Advances and Perspectives, ed. H. Eyring and D. Henderson, Academic Press, New York, 1980, vol. 5, p. 1.

29 L. Blum, J. N. Herrera and J. F. Rojas-Rodríguez, Rev. Mex. Fis., 1995, 41, 618.

30 C. W. Outhwaite, Chem. Phys. Lett., 1970, 5, 77.

31 C. W. Outhwaite and L. B. Bhuiyan, Condens. Matter Phys., 2019, 22, 23801.

32 R. Kjellander, J. Phys. Chem., 1995, 99, 10392.

33 D. G. Hall, Z. Phys. Chem., 1991, 174, 89.

34 B. P. Lee and M. E. Fisher, Phys. Rev. Lett., 1996, 76, 2906.

35 B. P. Lee and M. E. Fisher, Europhys. Lett., 1997, 39, 611.

36 L. M. Varela, M. Perez-Rodriguez, M. Garcia, F. Sarmiento and V. Mosquera, J. Chem. Phys., 1998, 109, 1930.

37 L. M. Varela, M. Perez-Rodriguez, M. García and V. Mosquera, J. Chem. Phys., 2000, 113, 292.

38 L. M. Varela, J. M. Ruso, M. García and V. Mosquera, J. Chem. Phys., 2000, 113, 10174.

39 L. M. Varela, M. García and V. Mosquera, Physica A, 2003, 323, 75 . 
40 M. Ding, Y. Liang, B.-S. Lu and X. Xing, J. Stat. Phys., 2016, 165, 970.

41 Y. Avni, R. M. Adar and D. Andelman, Phys. Rev. E, 2020, 101, 010601.

42 R. Triolo, J. R. Grigera and L. Blum, J. Chem. Phys., 1976, 80, 1858.

43 L. Blum and J. S. Høye, J. Chem. Phys., 1977, 81, 1311.

44 T. Sun, J.-L. Lénard and A. S. Teja, J. Phys. Chem., 1994, 98, 6870.

45 B. Larsen, G. Stell and K. C. Wu, J. Chem. Phys., 1977, 67, 530.

46 M. C. Abramo, C. Caccamo and G. Pizzimenti, J. Chem. Phys., 1983, 78, 357.

47 G. Stell and S. F. Sun, J. Chem. Phys., 1975, 63, 5333.

48 T. Xiao and X. Song, J. Chem. Phys., 2011, 135, 104104.

49 T. Xiao, Electrochim. Acta, 2015, 178, 101.

50 T. Xiao and X. Song, J. Chem. Phys., 2017, 146, 124118.

51 J. Rasaiah, Chem. Phys. Lett., 1970, 7, 260.

52 J. C. Rasiah, J. Chem. Phys., 1972, 56, 3071.

53 B. Larsen, J. Chem. Phys., 1978, 68, 4511.

54 P. Sloth and T. S. Sørensen, J. Phys. Chem., 1990, 94, 2116.

55 E. Gutiérrez-Valladares, M. Lukšič, B. Millán-Malo, B. Hribar-Lee and V. Vlachy, Condens. Matter Phys., 2011, 14, 33003.

56 T. L. Croxton and D. A. McQuarrie, J. Phys. Chem., 1979, 83, 1840.

57 T. Ichiye and A. D. J. Haymet, J. Chem. Phys., 1990, 93, 8954.

58 D.-M. Duh and A. D. J. Haymet, J. Chem. Phys., 1992, 97, 7716.

59 C. S. Babu and T. Ichiye, J. Chem. Phys., 1994, 100, 9147. 60 T.-H. Chung and L. L. Lee, J. Chem. Phys., 2009, 130, 134513.

$61 \mathrm{~J}$. W. Zwanikken, P. K. Jha and M. Olvera de la Cruz, J. Chem. Phys., 2011, 135, 064106.

62 D. M. Burley, V. C. L. Hutson and C. W. Outhwaite, Mol. Phys., 1972, 23, 867.

63 C. W. Outhwaite, M. Molero and L. B. Bhuiyan, J. Chem. Soc., Faraday Trans., 1993, 89, 1315.

64 A. O. Quiñones, L. B. Bhuiyan and C. W. Outhwaite, Condens. Matter Phys., 2018, 21, 23802.

65 M. Su, Z. Xu and Y. Wang, J. Phys.: Condens. Matter, 2019, 31, 355101.

66 A. L. Kholodenko and A. L. Beyerlein, Phys. Rev. A: At., Mol., Opt. Phys., 1986, 34, 3309.

67 D. di Caprio, J. Stafiej and J. P. Badiali, J. Chem. Phys., 1998, 108, 8572 .

68 R. R. Netz and H. Orland, Europhys. Lett., 1999, 45, 726.

69 R. R. Netz and H. Orland, Eur. Phys. J. E: Soft Matter Biol. Phys., 2000, 1, 67.

70 J.-M. Caillol, J. Stat. Phys., 2004, 115, 1461.

71 D. N. Card and J. P. Valleau, J. Chem. Phys., 1970, 52, 6232.

72 J. P. Valleau and L. K. Cohen, J. Chem. Phys., 1980, 72, 5935.

73 J. P. Valleau, L. K. Cohen and D. N. Card, J. Chem. Phys., 1980, 72, 5942.

74 W. van Megen and I. K. Snook, Mol. Phys., 1980, 39, 1043.

75 P. Sloth, T. S. Sørensen and J. B. Jensen, J. Chem. Soc., Faraday Trans. 2, 1987, 83, 881.

76 T. S. Sørensen, P. Sloth, H. B. Nielsen and J. B. Jensen, Acta Chem. Scand., Ser. A, 1988, 42, 237.
77 B. R. Svensson and C. E. Woodward, Mol. Phys., 1988, 64, 247.

78 Z. Abbas, E. Ahlberg and S. Nordholm, Fluid Phase Equilib., 2007, 260, 233.

79 Z. Abbas, E. Ahlberg and S. Nordholm, J. Phys. Chem. B, 2009, 113, 5905.

80 J. Janecek and R. R. Netz, J. Chem. Phys., 2009, 130, 074502.

81 R. Kjellander and J. Ulander, Mol. Phys., 1998, 95, 495.

82 A. McBride, M. Kohonen and P. Attard, J. Chem. Phys., 1998, 109, 2423.

83 B. Forsberg, J. Ulander and R. Kjellander, J. Chem. Phys., 2005, 122, 064502.

84 P. Attard, Phys. Rev. E: Stat., Nonlinear, Soft Matter Phys., 1993, 48, 3604.

85 N. Bjerrum, Kgl. Dan. Vidensk. Selsk. Mat.-Fys. Medd., 1926, 7, 1.

86 H. Yokoyama and H. Yamatera, Bull. Chem. Soc. Jpn., 1975, 48, 1770.

87 M. E. Fisher and Y. Levin, Phys. Rev. Lett., 1993, 71, 3826. 88 Y. Levin and M. E. Fisher, Physica A, 1996, 225, 164.

89 W. Ebeling and M. Grigo, Ann. Phys., 1980, 37, 21.

90 T. Cartailler, P. Turq, L. Blum and N. Condamine, J. Phys. Chem., 1992, 96, 6766.

91 Y. Zhou, S. Yeh and G. Stell, J. Chem. Phys., 1995, 102, 5785. 92 S. Yeh, Y. Zhou and G. Stell, J. Phys. Chem., 1996, 100, 1415.

93 D. M. Zuckerman, M. E. Fisher and B. P. Lee, Phys. Rev. E: Stat., Nonlinear, Soft Matter Phys., 1997, 56, 6569.

94 R. Kjellander, J. Chem. Phys., 2016, 145, 124503.

95 R. Kjellander, J. Chem. Phys., 2018, 148, 193701.

96 Z. Abbas, M. Gunnarsson, E. Ahlberg and S. Nordholm, J. Colloid Interface Sci., 2001, 243, 11.

97 N. F. Carnahan and K. E. Starling, J. Chem. Phys., 1969, 51, 635.

98 R. Kjellander, Soft Matter, 2019, 15, 5866.

99 M. A. Gebbie, A. M. Smith, H. A. Dobbs, A. A. Lee, G. G. Warr, X. Banquy, M. Valtiner, M. W. Rutland, J. N. Israelachvili, S. Perkin and R. Atkin, Chem. Commun., 2017, 53, 1214.

100 R. Kjellander and D. J. Mitchell, Chem. Phys. Lett., 1992, 200, 76.

101 R. Kjellander and D. J. Mitchell, J. Chem. Phys., 1994, 101, 603.

102 D. J. Mitchell and B. W. Ninham, Chem. Phys. Lett., 1978, 53, 397.

103 P. Kékicheff and B. W. Ninham, Europhys. Lett., 1990, 12, 471.

104 A. M. Smith, P. Maroni, G. Trefalt and M. Borkovec, J. Phys. Chem. B, 2019, 123, 1733.

105 B. Stojimirović, M. Galli and G. Trefalt, Phys. Rev. Res., 2020, 2, 023315.

106 R. Kjellander and R. Ramirez, J. Phys.: Condens. Matter, 2005, 17, S3409.

107 D. D. Carley, J. Chem. Phys., 1967, 46, 3783.

108 F. H. Stillinger and R. Lovett, J. Chem. Phys., 1968, 48, 3858.

109 L. Verlet and J.-J. Weis, Phys. Rev. A: At., Mol., Opt. Phys., 1972, 5, 939.

110 W. R. Smith, D. J. Henderson, P. J. Leonard, J. A. Barker and E. W. Grundke, Mol. Phys., 2008, 106, 3. 